DAVID-OLIVIER JAQUET

\title{
Domaines de Voronoï et algorithme de réduction des formes quadratiques définies positives
}

Séminaire de Théorie des Nombres de Bordeaux, tome 2, $\mathrm{n}^{\circ} 1$ (1990), p. $163-215$

<http://www.numdam.org/item?id=JTNB_1990_2_1_163_0>

(C) Université Bordeaux 1, 1990, tous droits réservés.

L'accès aux archives de la revue «Séminaire de Théorie des Nombres de Bordeaux » (http://jtnb.cedram.org/) implique l'accord avec les conditions générales d'utilisation (http://www.numdam.org/conditions). Toute utilisation commerciale ou impression systématique est constitutive d'une infraction pénale. Toute copie ou impression de ce fichier doit contenir la présente mention de copyright.

\section{Numdam}

Article numérisé dans le cadre du programme

Numérisation de documents anciens mathématiques

http://www.numdam.org/ 
Séminaire de Théorie des Nombres, Bordeaux 2 (1990), 163-215

\title{
Domaines de Voronoï et algorithme de réduction des formes quadratiques définies positives.
}

\author{
par DAVID-Olivier JAQUET
}

\begin{abstract}
Résumé - J'illustre la situation générale par un exemple simple, qui permet de mieux comprendre la géométrie de l'espace des domaines de Voronoï. Ensuite, je donne des résultats généraux sur les arêtes d'un domaine de Voronoï. Finalement, pour les représentants des 15 classes connues de formes parfaites à 7 variables, non équivalentes à $E_{7}$ et qui possèdent plus de 28 vecteurs minimaux, je fournis une description détaillée de leurs orbites de voisines.
\end{abstract}

Abstract - I illustrate the general situation by a simple example in order to understand better the geometry of the space of Voronoï's domains. Then I give general results about the edges of a Voronoi's domain. Finally, for the representatives of the 15 known classes of perfect forms in 7 variables which are non equivalent to $E_{7}$ and have more than 28 minimal vectors, $I$ provide a detailed description of their orbits of neighbours.

\section{Introduction}

Soit $Q$ une forme quadratique réelle à $n$ variables, définie positive. On peut exprimer cette forme de la manière suivante :

$Q(x)=x^{t} A x$ où $A$ est une matrice symétrique réelle définie positive.

Remarquons tout d'abord que $A$ peut être considérée comme la matrice des produits scalaires d'une base de $\mathbf{R}^{n}$. Restreindre $Q(x)$ à $x \in \mathbf{Z}^{n}$ revient à ne considérer que les combinaisons linéaires entières des vecteurs de cette base, c'est- à-dire les points du réseau engendré par les vecteurs de cette base. $Q(x)$ nous donne alors le carré de la distance euclidienne entre le point $x$ du réseau et l'origine.

On appelle minimum de $A$, noté min $A$, le minimum sur $\mathbf{Z}^{n} \backslash\{0\}$ de $Q(x)$. C'est donc le carré de la distance euclidienne à l'origine, du point du réseau le plus proche de 0 .

Les vecteurs minimaux de $A$ sont les paires de vecteurs $\pm v_{k} \in \mathbf{Z}^{n}$ $(k=1, \ldots, s)$, qui vérifient $v_{k}^{t} A v_{k}=\min A$.

Une forme est dite parfaite si la connaissance des coordonnées entières de ses vecteurs minimaux dans une base du réseau permet de retrouver la matrice des produits scalaires de cette base, c'est- à-dire $A$. 
Plaçons-nous maintenant dans l'espace des matrices symétriques réelles $n \times n$, qui est de dimension $N=\frac{n(n+1)}{2}$; on identifie cet espace à $\mathbf{R}^{N}$ qu'on rend euclidien via le produit scalaire $A \cdot B=$ trace $A B$.

J'appellerai cet espace l'espace des domaines de Voronoï ou plus simplement l'espace de Voronoï.

A chaque vecteur minimal $v_{k}$, on peut faire correspondre un point $a_{k}$ dans cet espace : on pose $a_{k}=v_{k} v_{k}^{t}$. (On remarque, au passage, que $a_{k} \cdot A=\min A$.)

A chaque $a_{k}$, on associe alors la demi-droite qui part de l'origine et contient $a_{k}$. L'enveloppe convexe de ces demi-droites est appelée domaine associé à $A$.

On montre facilement que $A$ est parfaite si et seulement si son domaine est de dimension maximale.

On appelle faces de dimension $N-1$ du domaine, les intersections de ce domaine avec ses hyperplans d'appui.

On appelle face de dimension $d(d<N-1)$ toute variété de dimension $d$ obtenue en intersectant des faces de dimension $N-1$.

Une arête du domaine est une face de dimension 1.

Définissons, maintenant, une action de $G L_{n}(\mathbf{Z})$ sur l'ensemble des formes quadratiques, en considérant tout élément de $G L_{n}(\mathbf{Z})$ comme une matrice de changement de base. On dira que deux formes quadratiques sont équivalentes si elles sont positivement proportionnelles à des formes appartenant à une même orbite. L'action définie ci-dessus induit une action de $G L_{n}(\mathbf{Z})$ sur l'ensemble des domaines de Voronoï. On dira que deux domaines sont équivalents s'ils appartiennent à la même orbite.

On montre facilement que deux domaines sont équivalents si et seulement si les formes parfaites correspondantes sont équivalentes.

Voronoï démontre plusieurs résultats. En particulier :

(i) Soit une face $F$ de dimension $N-1$ appartenant à un domaine. Alors $F$ appartient également à un autre domaine. Géométriquement, ces deux domaines se situent de part et d'autre de $F$. Ces deux domaines sont dits voisins ou contigus.

(ii) Toute forme quadratique définie positive appartient au moins à un domaine.

(iii) Soient deux domaines quelconques. Il existe un chemin reliant ces deux domaines qui, à chaque pas, ne fait que passer d'un domaine à un domaine voisin. 
(iv) A équivalence près, il n'existe qu'un nombre fini de formes parfaites en dimension $n$, donc qu'un nombre fini de domaines inéquivalents.

\section{Etude d'une situation particulière :}

J'aimerais reprendre les notions de formes parfaites et de domaines associés aux formes parfaites dans le cas des formes à 2 variables, c'est-à-dire $n=2$. L'espace des domaines de Voronoï est difficilement visualisable dans le cas général; seul le cas $n=2, N=3$ s'y prête bien. C'est pourquoi il est intéressant d'approfondir cette situation particulière dans l'optique de Voronoï, afin de mieux saisir la géométrie de l'espace de Voronoï.

Prenons, pour commencer, la matrice identité. Elle est associée à la forme $x^{2}+y^{2}$.

Son minimum vaut 1 . Ses vecteurs minimaux sont $\pm\left(\begin{array}{l}1 \\ 0\end{array}\right), \pm\left(\begin{array}{l}0 \\ 1\end{array}\right)$.

Cette forme n'est pas parfaite. En effet, il existe une infinité de formes quadratiques définies positives qui possèdent ces vecteurs comme vecteurs minimaux.

Une forme quadratique à deux variables est donnée par :

$$
\left(\begin{array}{ll}
x & y
\end{array}\right)\left(\begin{array}{ll}
a & b \\
b & c
\end{array}\right)\left(\begin{array}{l}
x \\
y
\end{array}\right)
$$

Si $\pm\left(\begin{array}{l}1 \\ 0\end{array}\right)$ est un vecteur minimal, alors $a=1$.

$\mathrm{Si} \pm\left(\begin{array}{l}0 \\ 1\end{array}\right)$ est un vecteur minimal, alors $c=1$.

Mais $b$ n'est pas déterminé. On montre facilement que pour tout $b$ inférieur en valeur absolue à $\frac{1}{2}$, la forme correspondante possède exactement ces vecteurs comme vecteurs minimaux. Par contre, lorsque $b$ vaut $\frac{1}{2}$, on obtient une forme qui possède un vecteur minimal supplémentaire. Cette forme est proportionnelle à $A_{2}=\left(\begin{array}{ll}2 & 1 \\ 1 & 2\end{array}\right)$. Elle est parfaite. En effet, le vecteur minimal supplémentaire $\pm\left(\begin{array}{c}1 \\ -1\end{array}\right)$ introduit la condition $1-2 b+1=1$, condition qui détermine $b$.

Le réseau correspondant à cette matrice est engendré par deux vecteurs de même longueur et formant un angle de $60^{\circ}$.

C'est le réseau, bien connu, d'empilement de sphères le plus dense dans le plan. 
fig. 1

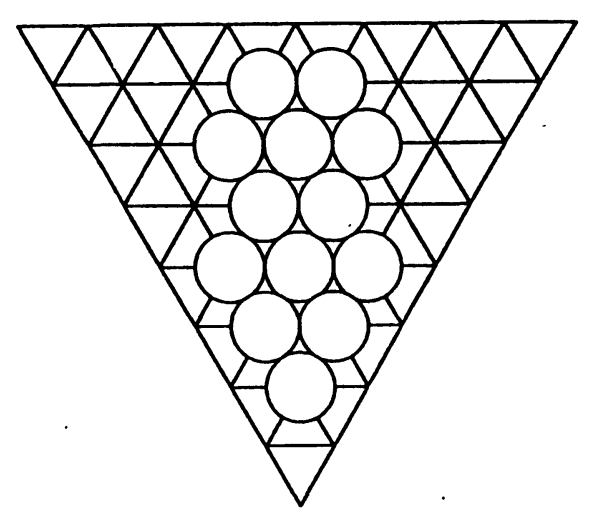

Dans $\mathbf{R}^{3}$, le domaine associé $\mathbf{a}\left(\begin{array}{ll}2 & 1 \\ 1 & 2\end{array}\right)$ possède exactement 3 arêtes :

$a_{1}=\left(\begin{array}{ll}1 \\ 0\end{array}\right)\left(\begin{array}{ll}1 & 0\end{array}\right)=\left(\begin{array}{ll}1 & 0 \\ 0 & 0\end{array}\right), a_{2}=\left(\begin{array}{ll}0 & 0 \\ 0 & 1\end{array}\right)$ et $a_{3}=\left(\begin{array}{cc}1 & -1 \\ -1 & 1\end{array}\right)$.

Comme $s=N=3$, on calcule les faces du domaine en enlevant à tour de rôle chacune des arêtes; celles qui restent déterminent une face. Par exemple, on obtient $F_{1}$ en retirant $a_{1}$ comme suit :

Posons $F_{1}=\left(\begin{array}{ll}f_{1} & f_{2} \\ f_{2} & f_{3}\end{array}\right)$, un vecteur normal à la face cherchée. Donc $F_{1}$ est normal à $a_{2}$ et à $a_{3}$. Ces conditions s'expriment à l'aide du produit scalaire donné par la trace.

(i) $F_{1} \cdot a_{2}=\operatorname{trace}\left(F_{1} a_{2}\right)=0$,

(ii) $F_{1} \cdot a_{3}=\operatorname{trace}\left(F_{1} a_{3}\right)=0$.

La condition (i) nous donne $f_{3}=0$; la condition (ii) implique que $f_{1}-2 f_{2}+f_{3}=0$. Ces équations définissent $F_{1}$ à un facteur près. On peut choisir $F_{1}$ de telle manière que ses composantes soient entières et premières entre elles, et fixer le sens de $F_{1}$ en demandant que $\operatorname{trace}\left(F_{1} a_{1}\right)>0$. On obtient alors $F_{1}=\left(\begin{array}{ll}2 & 1 \\ 1 & 0\end{array}\right)$.

De manière analogue, on détermine $F_{2}=\left(\begin{array}{ll}0 & 1 \\ 1 & 2\end{array}\right)$ et $F_{3}=\left(\begin{array}{cc}0 & -1 \\ -1 & 0\end{array}\right)$. 
Etudions la voisine de $\left(\begin{array}{ll}2 & 1 \\ 1 & 2\end{array}\right)$ relativement à la face $\left(\begin{array}{cc}0 & -1 \\ -1 & 0\end{array}\right)$. Cette voisine peut s'écrire $\left(\begin{array}{ll}2 & 1 \\ 1 & 2\end{array}\right)+\rho\left(\begin{array}{cc}0 & -1 \\ -1 & 0\end{array}\right)$, pour un certain $\rho>0$. La voisine étant unique par Voronoï, il n'existe qu'une seule valeur de $\rho$ positive qui donne une forme parfaite de minimum 2. On devine que pour $\rho=2$, la forme correspondante $\left(\begin{array}{cc}2 & -1 \\ -1 & 2\end{array}\right)$ est parfaite. C'est la voisine cherchée. La figure ci-dessous (fig. 2) visualise, dans $\mathbf{R}^{3}$, les domaines associés à ces deux formes voisines. Les domaines correspondants sont voisins. Leur face commune admet comme vecteur normal $\left(\begin{array}{cc}0 & -1 \\ -1 & 0\end{array}\right)$. Pour plus de clarté, j'ai indiqué en petits caractères les arêtes des domaines et en caractères gras les formes correspondant à chacun de ces domaines. On prolongera par l'esprit les faces dont je n'ai dessiné qu'une portion.

fig. 2

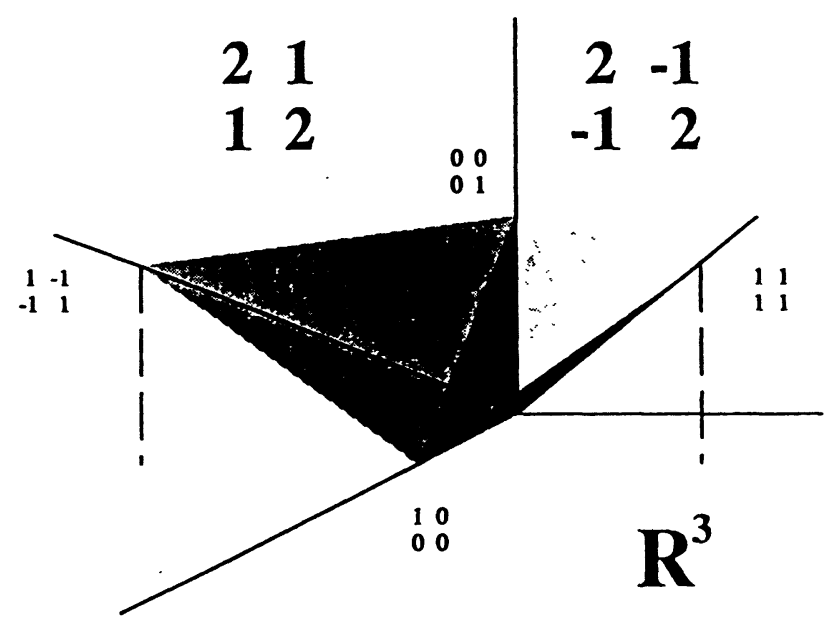

En contemplant $\left(\begin{array}{ll}2 & 1 \\ 1 & 2\end{array}\right)=\left(\begin{array}{cc}1 & 0 \\ 0 & -1\end{array}\right)^{t}\left(\begin{array}{cc}2 & -1 \\ -1 & 2\end{array}\right)\left(\begin{array}{cc}1 & 0 \\ 0 & -1\end{array}\right)$, on conclut que ces deux formes sont équivalentes.

On peut de même calculer les voisines correspondant aux deux autres faces. La voisine correspondant à $F_{1}$ est donnée par $\left(\begin{array}{ll}6 & 3 \\ 3 & 2\end{array}\right)$, celle cor- 
respondant à $F_{2} \operatorname{par}\left(\begin{array}{ll}2 & 3 \\ 3 & 6\end{array}\right)$. Il est facile de voir que ces formes sont équivalentes à $A_{2}$ soit en explicitant la matrice de changement de base, soit en constatant que le groupe des automorphismes de $A_{2}$ est transitif sur les faces du domaine associé à $A_{2}$. Il faut comprendre automorphisme dans le sens d'isométrie de la forme à coefficients entiers. Voronoï démontre que, de manière générale, le groupe des automorphismes de $A_{n}$ ( $A_{n}$ est la forme parfaite donnée par $\left.x_{1}^{2}+x_{2}^{2}+\ldots+x_{n}^{2}+\left(x_{1}+x_{2}+\ldots+x_{n}\right)^{2}\right)$ est transitif sur les faces du domaine associé à $A_{n}$.

Dans les cas $n=2$ et $n=3$, les voisines de $A_{n}$ sont équivalentes à $A_{n}$. $\Pi$ n'existe donc, d'après Voronoï, qu'une seule classe de formes parfaites dans ces dimensions.

Revenons au cas $n=2$. Après avoir calculé les voisines de $A_{2}$, on peut déterminer les voisines des voisines de $A_{2}$, etc.

La figure ci-dessous prolonge la figure 2. Les arêtes de tout domaine sont de la forme $\left(\begin{array}{l}a \\ b\end{array}\right)\left(\begin{array}{ll}a & b\end{array}\right)=\left(\begin{array}{cc}a^{2} & a b \\ a b & b^{2}\end{array}\right)$. Une telle arête sera représentée dans $\mathbf{R}^{3}$ par $\left(a^{2}, \sqrt{2} a b, b^{2}\right)$. Ce sont ses coordonnées dans la base orthonormée $\left(\begin{array}{ll}1 & 0 \\ 0 & 0\end{array}\right), \frac{1}{\sqrt{2}}\left(\begin{array}{ll}0 & 1 \\ 1 & 0\end{array}\right),\left(\begin{array}{ll}0 & 0 \\ 0 & 1\end{array}\right)$.

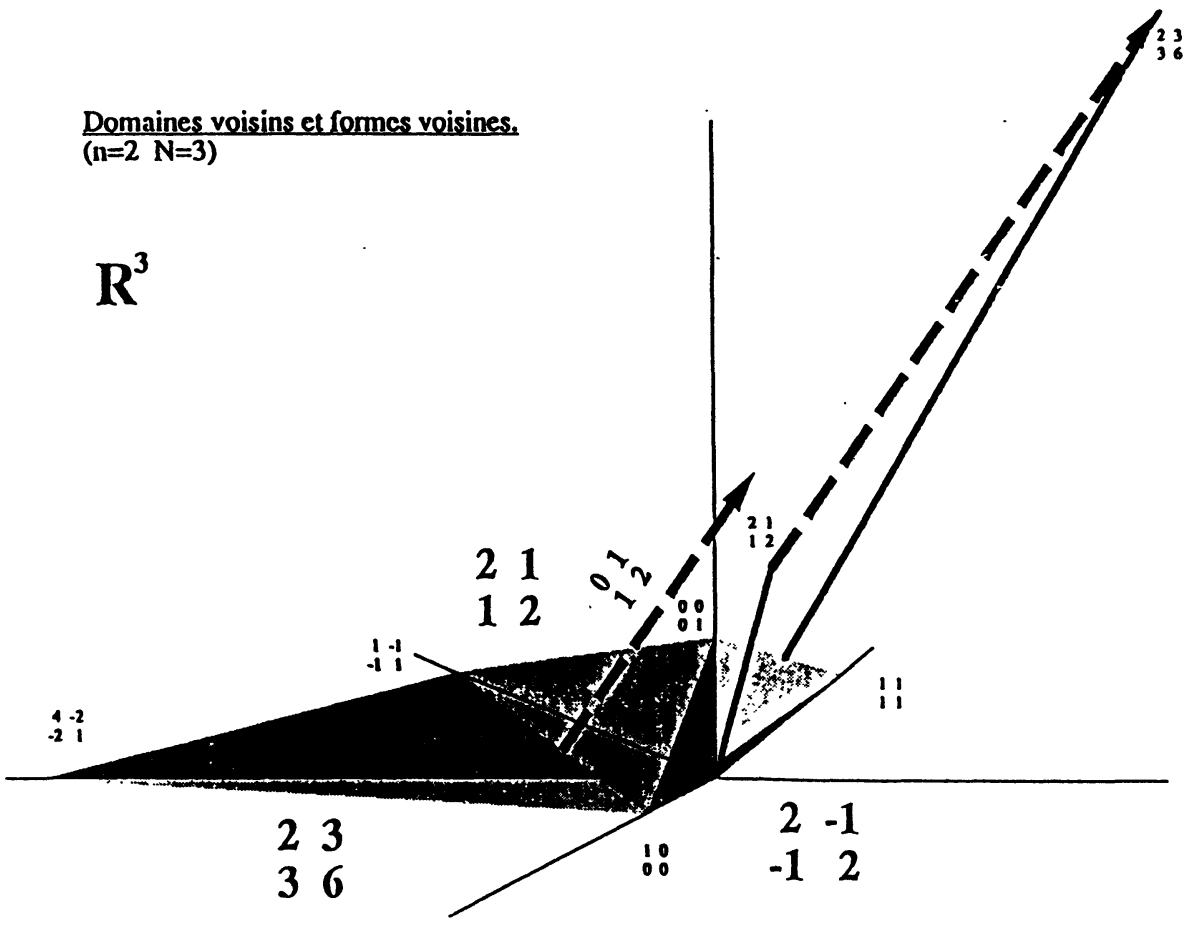


En remarquant que $(\sqrt{2} a b)^{2}=2 a^{2} b^{2}$, on conclut que toutes les arêtes appartiennent à la surface d'équation $y^{2}=2 x z$. II s'agit d'un cône de révolution dont le sommet est à l'origine. La section de ce cône par le plan d'équation $x+z=2$ est un cercle de rayon $\sqrt{2}$ centré en $(1,0,1)$. On remarquera au passage que $(1,0,1)$ correspond à la matrice identité. Ce tableau met en évidence l'aspect fractal des domaines de Voronoï. Autre propriété intéressante : dans ce cas particulier, le chemin reliant deux domaines est essentiellement unique. En effet, associons à chaque domaine un point intérieur et relions les points qui appartiennent à deux domaines voisins. Le graphe ainsi obtenu est en fait un arbre.

fig. 4

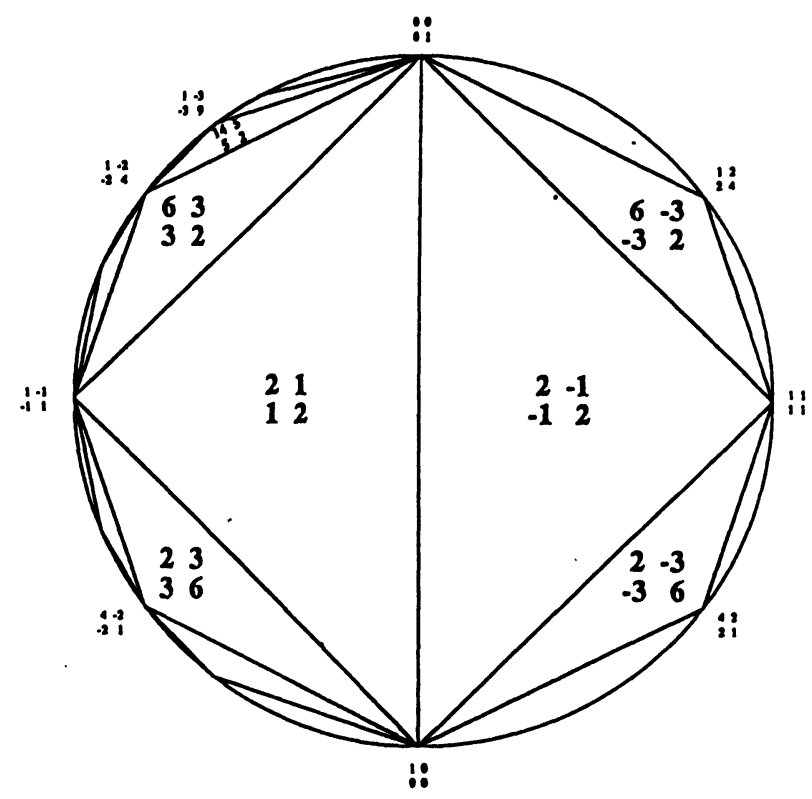

Coupe selon le plan d'equation $x+z=2$.

Le sens d'un vecteur normal à une face d'un domaine est fixé par la condition que toutes les arêtes de ce domaine ont un produit scalaire positif ou nul avec ce vecteur. Pour qu'une forme $B$ soit intérieure à un domaine, il faut et il suffit que $F \cdot B>0$ pour tout $F$, vecteur normal à une face du domaine. On voit aisément que $A_{2}$ n'est pas intérieure à son domaine. En effet, trace $\left(\begin{array}{cc}0 & -1 \\ -1 & 0\end{array}\right)\left(\begin{array}{ll}2 & 1 \\ 1 & 2\end{array}\right)<0$. Par contre, en additionnant les 
arêtes d'un domaine, on obtient toujours une forme intérieure à ce domaine. Ici, dans chaque cas, on trouve la forme adjointe. Par exemple, la forme adjointe de $\left(\begin{array}{ll}2 & 1 \\ 1 & 2\end{array}\right)$ est $\left(\begin{array}{cc}2 & -1 \\ -1 & 2\end{array}\right)$ :

$$
\left(\begin{array}{cc}
2 & -1 \\
-1 & 2
\end{array}\right)=\left(\begin{array}{ll}
1 & 0 \\
0 & 0
\end{array}\right)+\left(\begin{array}{cc}
1 & -1 \\
-1 & 1
\end{array}\right)+\left(\begin{array}{ll}
0 & 0 \\
0 & 1
\end{array}\right) \text {. }
$$

On dit que $A_{2}$ est eutactique. (Sa forme adjointe est intérieure à son domaine.) Je rappellerai ici le critère, maintenant classique, démontré par Voronoï : une forme est extrême si et seulement si elle est parfaite et eutactique.

fig. 5

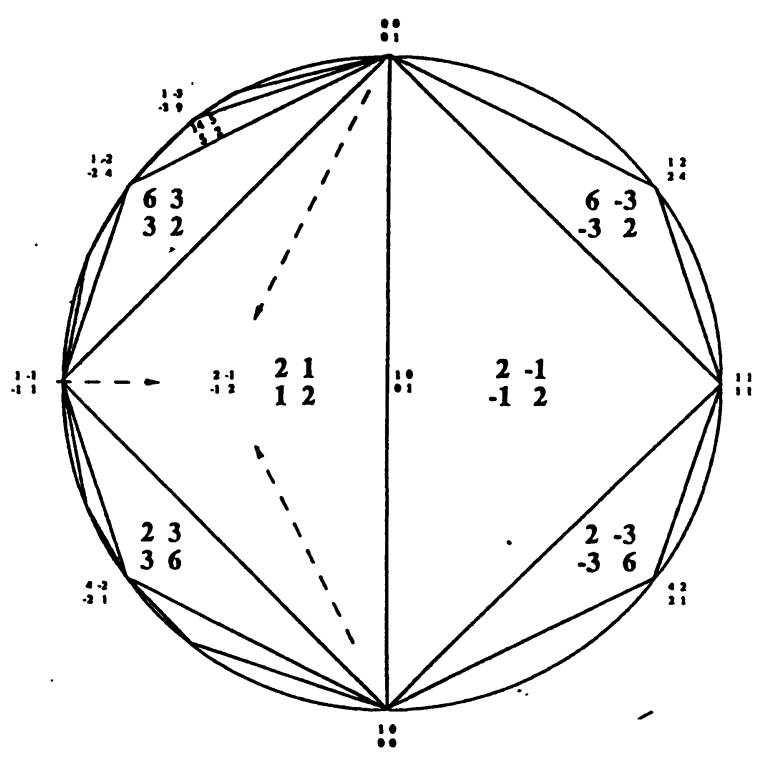

Coupe selon le plan d'équation $x+z=2$.

Lorsqu'on a une forme parfaite, on peut représenter son domaine dans l'espace de Voronoï; mais on peut aussi la considérer comme un point dans cet espace. On vient de voir qu'en général ce point n'appartient pas au domaine de la forme. Quel est alors le lien géométrique entre deux domaines voisins et les deux formes parfaites voisines correspondantes?

J'aimerais répondre à cette question par un exemple. Partons du domaine 
associé à $A_{2}$ et considérons la face de ce domaine, donnée par $\left(\begin{array}{ll}0 & 1 \\ 1 & 2\end{array}\right)$. Cette face appartient également à un autre domaine : le domaine associé $\grave{a}\left(\begin{array}{ll}2 & 3 \\ 3 & 6\end{array}\right)$. Ces deux domaines sont donc voisins et les formes parfaites correspondantes sont voisines. Voronoï nous dit que $\left(\begin{array}{ll}2 & 3 \\ 3 & 6\end{array}\right)$ doit s'obtenir en additionnant à $\left(\begin{array}{ll}2 & 1 \\ 1 & 2\end{array}\right)$ un multiple positif du vecteur normal à la face, c'est-à-dire un multiple positif de $\left(\begin{array}{ll}0 & 1 \\ 1 & 2\end{array}\right)$. Numériquement, on obtient :

$$
\left(\begin{array}{ll}
2 & 3 \\
3 & 6
\end{array}\right)=\left(\begin{array}{ll}
2 & 1 \\
1 & 2
\end{array}\right)+2\left(\begin{array}{ll}
0 & 1 \\
1 & 2
\end{array}\right)
$$

La figure suivante illustre cette relation.

fig. 6

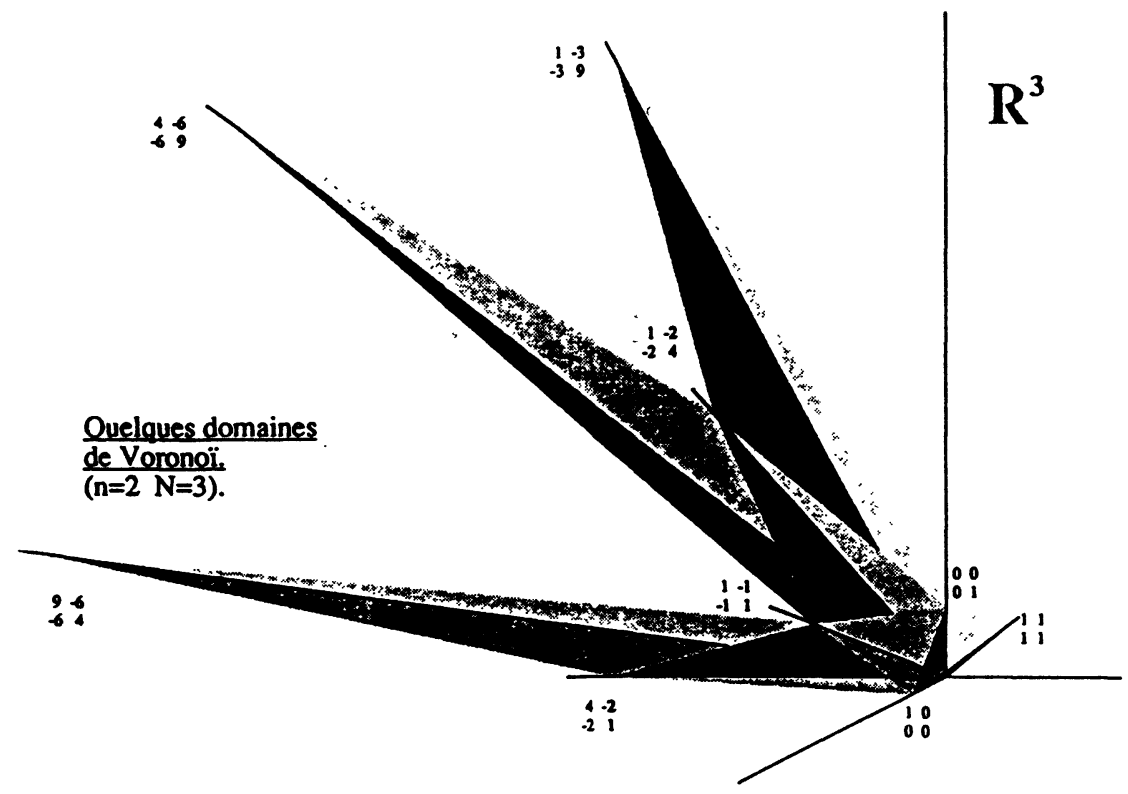

Essayons maintenant de comprendre le procédé de réduction des formes quadratiques définies positives, donné par Voronoï.

Le procédé se base sur la propriété suivante :

( $\star$ S Soit $f$ une forme quadratique définie positive. Pour tout nombre $\beta$ 
fixé, il n'existe qu'un nombre fini de formes parfaites $A$ de minimum 1 satisfaisant $f \cdot A \leq \beta$.

Soit $f$ une forme quadratique définie positive. On sait que $f$ appartient au moins à un domaine de Voronoï. On peut même donner un procédé qui permet d'exhiber un tel domaine. En effet, partons d'un domaine quelconque de Voronoï. Si $f$ appartient à ce domaine, on a terminé l'opération. Sinon, $f$ est du mauvais côté d'une face au moins de ce domaine. Appelons $F$ la normale à cette face, $B$ la forme parfaite de minimum 1 associée à ce domaine et $C$ la voisine de $B$ relativement à $F$. Le minimum de $C$ est égal au minimum de $B$ et vaut par conséquent 1 . Si $f$ est du mauvais côté de la face correspondant à $F$, alors $F \cdot f<0$. On en déduit facilement que $f \cdot C<f \cdot B$. En répétant l'opération, on voit que le produit scalaire de $f$ avec la forme associée au domaine testé ne fait que décroître. La propriété ( ) garantit qu'après un nombre fini d'itérations on arrive dans un domaine contenant $f$.

Prenons par exemple pour $f$ la forme qui a pour matrice

$$
\left(\begin{array}{cc}
5 & 7 \\
7 & 12
\end{array}\right)=\left(\begin{array}{ll}
0 & 0 \\
0 & 1
\end{array}\right)+2\left(\begin{array}{ll}
1 & 2 \\
2 & 4
\end{array}\right)+3\left(\begin{array}{ll}
1 & 1 \\
1 & 1
\end{array}\right)
$$

Cette forme est intérieure au domaine associé à $\left(\begin{array}{cc}6 & -3 \\ -3 & 2\end{array}\right)$. Partons du domaine associé à $\left(\begin{array}{ll}2 & 1 \\ 1 & 2\end{array}\right)$. Puisque la trace de $\left(\begin{array}{cc}5 & 7 \\ 7 & 12\end{array}\right)\left(\begin{array}{cc}0 & -1 \\ -1 & 0\end{array}\right)$ est négative, on passe dans le domaine voisin relativement à $\left(\begin{array}{cc}0 & -1 \\ -1 & 0\end{array}\right)$, domaine associé à $\left(\begin{array}{cc}2 & -1 \\ -1 & 2\end{array}\right)$. (Voir fig. 4.) Notre forme de départ n'appartient pas à ce nouveau domaine. En effet, elle est du mauvais côté de la face qui relie le domaine associé à $\left(\begin{array}{cc}2 & -1 \\ -1 & 2\end{array}\right)$ avec le domaine associé à $\left(\begin{array}{cc}6 & -3 \\ -3 & 2\end{array}\right)$. En traversant cette face, on atteint le domaine contenant la forme de départ.

L'idée de Voronoï est la suivante :

Après la première opération, on est placé dans le domaine associé à $\left(\begin{array}{cc}2 & -1 \\ -1 & 2\end{array}\right)$. Comme $\left(\begin{array}{cc}2 & -1 \\ -1 & 2\end{array}\right)$ est équivalente à $\left(\begin{array}{ll}2 & 1 \\ 1 & 2\end{array}\right)$, les domaines associés à ces deux formes sont équivalents. $\mathrm{Si} S$ donne l'équivalence entre $\left(\begin{array}{cc}2 & -1 \\ -1 & 2\end{array}\right)$ et $\left(\begin{array}{ll}2 & 1 \\ 1 & 2\end{array}\right),\left(S^{-1}\right)^{t}$ transforme le domaine associé à 
$\left(\begin{array}{cc}2 & -1 \\ -1 & 2\end{array}\right)$ en le domaine associé à $\left(\begin{array}{ll}2 & 1 \\ 1 & 2\end{array}\right)$. En appliquant $\left(S^{-1}\right)^{t}$ à tous les domaines, on ramène le problème qui consistait à réduire $f$ à partir du domaine associé à $\left(\begin{array}{cc}2 & -1 \\ -1 & 2\end{array}\right)$, au problème qui consiste à réduire $S^{-1} f\left(S^{-1}\right)^{t}$ à partir du domaine associé à $\left(\begin{array}{ll}2 & 1 \\ 1 & 2\end{array}\right)$. En répétant ce processus, on obtient après un nombre fini d'itérations une forme $f^{\prime}$ équivalente à $f$ et intérieure au domaine associé à $\left(\begin{array}{ll}2 & 1 \\ 1 & 2\end{array}\right)$.

Notre exemple est illustré par la figure 7.

fig. 7

Illustration de la méthode de réduction des formes

quadratiques définies positives, décrite par Voronoi..

(n=2 $N=3$ ).

Forme de départ: $\begin{array}{ll}5 & 7 \\ 7 & 12\end{array} \quad$ Forme reduite: $\left[\begin{array}{rr}3 & -2 \\ -2 & 3\end{array}\right.$

(ii)

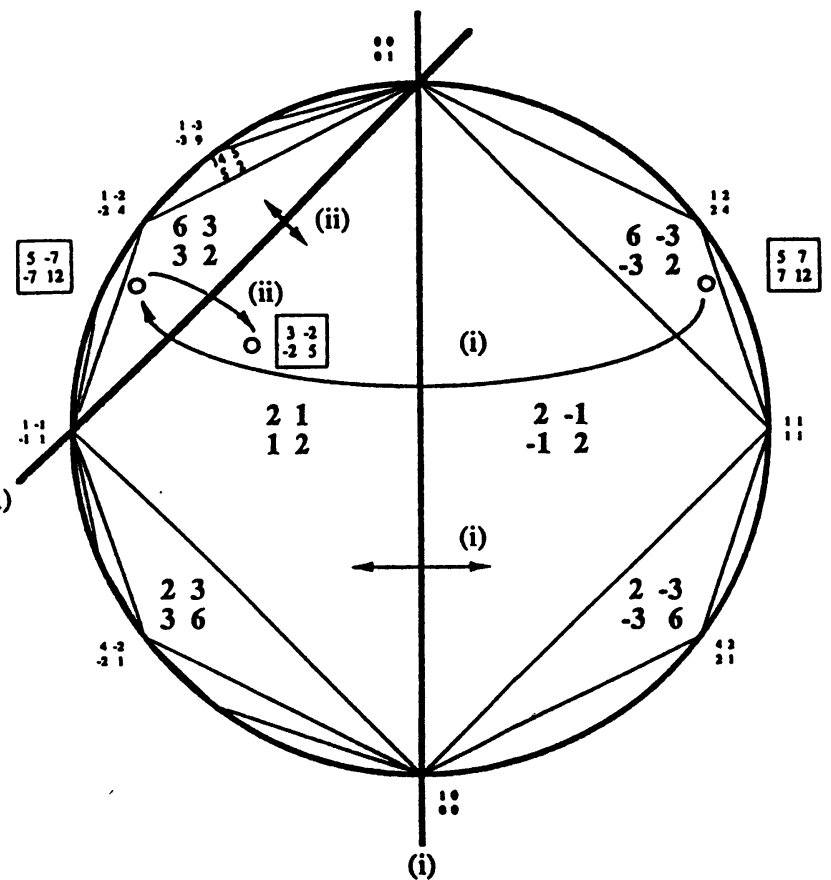

Coupe selon le plan d'équation $x+z=2$. 
Lorsqu'on se trouve, après la première opération, dans le domaine associé à $\left(\begin{array}{cc}2 & -1 \\ -1 & 2\end{array}\right)$, on peut prendre $S=\left(\begin{array}{cc}1 & 0 \\ 0 & -1\end{array}\right)$. En appliquant $\left(S^{-1}\right)^{t}$ aux domaines de Voronoï, le domaine associé à $\left(\begin{array}{cc}2 & -1 \\ -1 & 2\end{array}\right)$ est alors transformé en le domaine associé à $\left(\begin{array}{ll}2 & 1 \\ 1 & 2\end{array}\right)$, celui associé à $\left(\begin{array}{cc}6 & -3 \\ -3 & 2\end{array}\right)$ est envoyé sur le domaine associé à $\left(\begin{array}{ll}6 & 3 \\ 3 & 2\end{array}\right)$, etc. ; la forme $f$ donnée par $\left(\begin{array}{cc}5 & 7 \\ 7 & 12\end{array}\right)$ devient $\left(\begin{array}{cc}5 & -7 \\ -7 & 12\end{array}\right)$, intérieure au domaine associé à $\left(\begin{array}{ll}6 & 3 \\ 3 & 2\end{array}\right)$. Ensuite, la, réduction de la nouvelle forme $\left(\begin{array}{cc}5 & -7 \\ -7 & 12\end{array}\right)$ nous fait passer du domaine associé à $\left(\begin{array}{ll}2 & 1 \\ 1 & 2\end{array}\right)$ dans celui associé à $\left(\begin{array}{ll}6 & 3 \\ 3 & 2\end{array}\right)$, que l'on ramène sur le domaine associé à $\left(\begin{array}{ll}2 & 1 \\ 1 & 2\end{array}\right)$ grâce à l'équivalence entre $\left(\begin{array}{ll}6 & 3 \\ 3 & 2\end{array}\right)$ et $\left(\begin{array}{ll}2 & 1 \\ 1 & 2\end{array}\right)$ donnée, par exemple, par $S=\left(\begin{array}{cc}0 & 1 \\ 1 & -1\end{array}\right)$. En effet, $S^{t}\left(\begin{array}{ll}6 & 3 \\ 3 & 2\end{array}\right) S=$ $\left(\begin{array}{ll}2 & 1 \\ 1 & 2\end{array}\right)$. Pour calculer $f^{\prime}$, on utilisera donc $\left(S^{-1}\right)^{t}=\left(\begin{array}{ll}1 & 1 \\ 1 & 0\end{array}\right) ; f^{\prime}$ a pour matrice

$$
\left(\begin{array}{ll}
1 & 1 \\
1 & 0
\end{array}\right)^{t}\left(\begin{array}{cc}
5 & -7 \\
-7 & 12
\end{array}\right)\left(\begin{array}{ll}
1 & 1 \\
1 & 0
\end{array}\right)=\left(\begin{array}{cc}
3 & -2 \\
-2 & 5
\end{array}\right)
$$

Dans le cas général, pour appliquer cet algorithme de réduction, il suffit de connaître une liste exhaustive de formes parfaites non équivalentes, les faces et les voisines de toutes ces formes ainsi que les matrices de changement de base permettant d'identifier chacune de ces voisines à une des formes de la liste. L'intérêt de cet algorithme réside dans le fait qu'il ne demande pas de connaître explicitement le domaine (ou un domaine) qui contient la forme de départ.

Dans le cas $n=2$, on peut améliorer la réduction en pavant le domaine associé à $\left(\begin{array}{ll}2 & 1 \\ 1 & 2\end{array}\right)$ avec six régions (fig. 8 ) sur lesquelles le groupe des automorphismes de la forme agit transitivement.

En choisissant une région $\mathbf{R}$, on peut alors affiner le procédé de réduction : toute forme quadratique appartenant au domaine associé à $\left(\begin{array}{ll}2 & 1 \\ 1 & 2\end{array}\right)$ possède 
une forme équivalente dans $\mathbf{R}$. Donc, toute forme quadratique définie positive $f$, possède une forme $f^{\prime}$ équivalente dans $\mathbf{R}$.

$A$ un signe près et pour un bon choix de $\mathbf{R}$, la forme réduite satisfait alors les célèbres inégalités de Lagrange.

On peut généraliser ce procédé pour $n=3$.

fig. 8

\section{Exemple de région fondamentale}

dans le domaine associéz $\mathrm{A}_{2}$

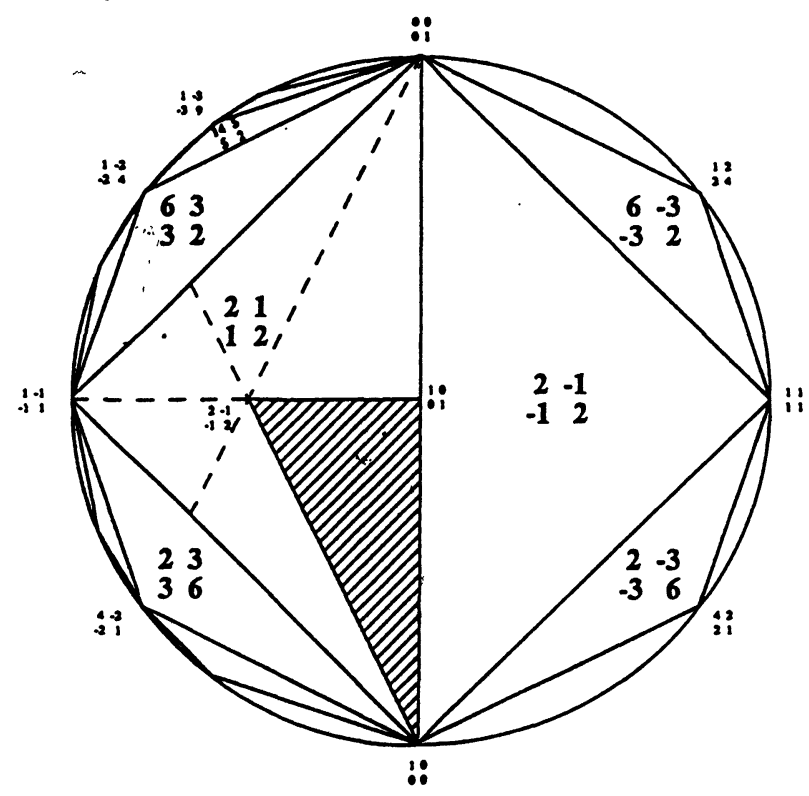

\section{Sur les arêtes d'un domaine de Voronoï :}

On montre aisément que le domaine d'une forme parfaite est de dimension maximale, mais on peut préciser certaines propriétés géométriques d'un tel domaine.

A priori, l'enveloppe convexe de demi-droites issues de l'origine peut 
définir plusieurs types de domaines :

(1) un domaine sans face, c'est-à-dire tout l'espace,

(2) un domaine pour lequel l'intersection de toutes les faces donne une variété de dimension $\geq 1$,

(3) un domaine "pyramidal", c'est-à-dire pour lequel l'intersection de toutes les faces se réduit à un point : l'origine.

Remarque : Un domaine de type (1) ou de type (2) pour lequel l'intersection de toutes les faces donne une variété de dimension $>1$, ne possède pas d'arêtes.

Par le biais de la notion, judicieusement introduite, de domaines corrélatifs, Voronoï , dans [4], démontre que ses domaines possèdent des faces et que seule l'origine appartient à toutes les faces d'un domaine. Par conséquent, tout domaine associé à une forme parfaite possède des arêtes.

Géométriquement, les demi-droites associées aux $a_{k}=v_{k} v_{k}^{t}$ et dont on prend l'enveloppe convexe sont donc suffisamment resserrées.

Voronoï montre que toute arête d'un domaine associé à une forme parfaite $A$, est une des demi-droites dont on a pris l'enveloppe convexe. J'affirme que la réciproque est vraie. Chaque demi-droite associée à $a_{k}=v_{k} v_{k}^{t}$ (où $v_{k}$ est un vecteur minimal de $A$ ) est une arête du domaine associé à $A$.

Contrairement aux apparences, Voronoï ne démontre pas cette deuxième partie : il admet, sans le démontrer, qu'aucun des $a_{j}$ ne peut s'exprimer comme combinaison linéaire à coefficients positifs ou nuls des autres $a_{k}$. Voici l'esquisse d'une démonstration :

Remarquons, d'abord, que tout $a_{k}$ fait un angle constant avec la matrice identité. Mieux, tout $a=v v^{t}$ avec $v \in \mathbb{R}^{n}$ fait un angle constant avec la matrice identité. On considère, bien entendu, la métrique induite par la trace. Cet angle satisfait $\cos \Phi=\frac{1}{\sqrt{n}}$. En particulier, $\left.\Phi \in\right] 0, \frac{\pi}{2}[$.

Cette remarque généralise la propriété constatée dans l'exemple $n=2$, exemple où toutes les arêtes des domaines de Voronoï se situaient sur la surface d'un cône de révolution dont l'axe passait pảr la matrice identité.

Considérons $\mathbf{C}$, l'ensemble des matrices réelles symétriques formant avec la matrice identité un angle $\leq \Phi$. On montrera facilement que $\mathbf{C}$ est un cône convexe et qu'une matrice symétrique est intérieure à $\mathbf{C}$ si et seulement si l'angle qu'elle forme avec la matrice identité est strictement inférieur à $\Phi$.

On en déduira que la somme de deux éléments de $\mathbf{C}$, non proportionnels, fournit toujours un point intérieur de $\mathbf{C}$.

Finalement, on conclura aisément en remarquant que $v v^{t}\left(v \in \mathbf{R}^{n}\right)$ appar- 
tient au bord de $\mathbf{C}$ et qu'une combinaison linéaire à coefficients positifs de $v_{i} v_{i}^{t}\left(v_{i} \in \mathbf{R}^{n}\right)$, donne un point intérieur de $\mathbf{C}$, dès que cette combinaison linéaire contient plus d'un terme!

Un domaine de Voronoï a été défini comme l'enveloppe convexe d'un ensemble bien déterminé de demi-droites. Par ce qui précède, on constate que cet ensemble de demi-droites possède une propriété géométrique de minimalité, à savoir : si l'on retire une demi-droite de cet ensemble, l'enveloppe convexe de celles qui restent est toujours modifiée.

4. Résultats actuels en dimension $n=7(N=28)$ :

L'intérêt tout particulier porté à cette dimension peut s'expliquer ainsi :

$n=3$ Le problème a été résolu par Gauss en 1831. Gauss a montré que $A_{3}$ représente la seule forme parfaite à équivalence près.

$n=4$,

$n=5$ Ces deux dimensions on été traitées en 1877 par Korbine et Zolotareff.

$n=6$ Il a fallu attendre 1957 pour que Barnes donne la liste exhaustive des formes parfaites à 6 variables, toujours à équivalence près.

$n=7$ Jusqu'en 1971, on ne connaissait que 22 formes parfaites non équivalentes en dimension $n=7$. Stacey ramena cette liste à 33 en employant des méthodes dues à Watson.

Dans le cadre de la thèse de doctorat effectuée sous la direction du Professeur François Sigrist, je cherche à montrer que cette liste de 33 formes en dimension $n=7$ est exhaustive. Il suffit pour cela, d'après Voronoï , de montrer que chacune des formes contiguës à l'une de ces 33 formes, est équivalente justement à une de ces 33 formes.

On trouvera la description détaillée des orbites de voisines pour les formes qui possèdent exactement 28 vecteurs minimaux dans [1] à quelques corrections mineures signalées dans [2].

Je remercie Conway et Sloane pour le précieux recensement qu'ils ont effectué dans [1] des formes parfaites connues en dimension $n \leq 7$. La description complète qu'ils nous donnent des groupes d'automorphismes de chacune de ces formes m'a également été d'une grande utilité.

Les résultats qui suivent concernent donc toutes les formes parfaites connues à 7 variables, qui ont plus de 28 vecteurs minimaux, excepté $E_{7}$ pour laquelle les calculs sont encore en cours. On trouvera, pour chaque orbite de voisines, sa cardinalité et l'identification de ses éléments avec une des 33 formes de la liste donnée par Conway et Sloane [1].

Pour les formes qui ont 34 ou 36 vecteurs minimaux, je donne explicite- 
ment, pour chaque orbite de voisines, un représentant de l'orbite et la matrice dans $G L_{n}(\mathbf{Z})$ qui permet l'identification de ce représentant avec une des 33 formes de la liste de Conway et Sloane.

Ces résultats sont reproduits directement à partir d'un listing d'ordinateur afin d'éviter les erreurs de transcription.

Pour $D_{7}$, qui possède 42 vecteurs minimaux, les résultats sont expliqués dans [2]. 
Forme parfaite $P(7,1)$.

$\begin{array}{lllllll}2 & 1 & 1 & 1 & 1 & 1 & 0 \\ 1 & 2 & 1 & 1 & 1 & 1 & 0 \\ 1 & 1 & 2 & 1 & 1 & 1 & 1 \\ 1 & 1 & 1 & 2 & 1 & 1 & 1 \\ 1 & 1 & 1 & 1 & 2 & 1 & 1 \\ 1 & 1 & 1 & 1 & 1 & 2 & 1 \\ 0 & 0 & 1 & 1 & 1 & 1 & 2\end{array}$

Nombre de vecteurs minimaux : 63

Minimum

$: 2$

Déterminant

$: 2$

Déterminant (minimum normalisé à 2) : 2

Ordre du groupe des automorphismes : 2903040

Calcul des orbites des voisines en cours.

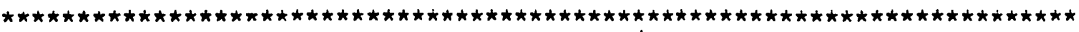
Forme parfaite $P(7,3)$.

$\begin{array}{lllllll}4 & 1 & 2 & 2 & 2 & 2 & 2 \\ 1 & 4 & 2 & 2 & 2 & 2 & 2 \\ 2 & 2 & 4 & 1 & 1 & 2 & 2 \\ 2 & 2 & 1 & 4 & 1 & 2 & 2 \\ 2 & 2 & 1 & 1 & 4 & 2 & 2 \\ 2 & 2 & 2 & 2 & 2 & 4 & 1 \\ 2 & 2 & 2 & 2 & 2 & 1 & 4\end{array}$

Nombre de vecteurs minimaux : 36

Minimum

4

Déterminant

486

Déterminant (minimum normalisé à 2) : 243/64

Ordre du groupe des automorphismes : 2592

Liste des orbites des voisines de la forme $\mathrm{P}(7,3)$ :

$1 \rightarrow$

Voisine = $\begin{array}{lllllll}4 & 2 & 2 & 2 & 2 & 2 & 2 \\ 2 & 4 & 2 & 2 & 2 & 2 & 2 \\ 2 & 2 & 4 & 2 & 0 & 2 & 2 \\ 2 & 2 & 2 & 4 & 0 & 2 & 2 \\ 2 & 2 & 0 & 0 & 4 & 2 & 2 \\ 2 & 2 & 2 & 2 & 2 & 4 & 2 \\ 2 & 2 & 2 & 2 & 2 & 2 & 4\end{array}$

$2 \rightarrow$

voisine = $\begin{array}{lllllll}4 & 0 & 2 & 0 & 2 & 2 & 0 \\ 0 & 4 & 0 & 2 & 2 & 0 & 2 \\ 2 & 0 & 4 & 0 & 0 & 2 & 0 \\ 0 & 2 & 0 & 4 & 0 & 2 & 2 \\ 2 & 2 & 0 & 0 & 4 & 0 & 2 \\ 2 & 0 & 2 & 2 & 0 & 4 & 0 \\ 0 & 2 & 0 & 2 & 2 & 0 & 4\end{array}$

$3 \rightarrow$

Voisine = $\begin{array}{lllllll}4 & 0 & 2 & 2 & 2 & 0 & 2 \\ 0 & 4 & 2 & 2 & 2 & 2 & 2 \\ 2 & 2 & 4 & 2 & 2 & 2 & 2 \\ 2 & 2 & 2 & 4 & 2 & 2 & 2 \\ 2 & 2 & 2 & 2 & 4 & 2 & 2 \\ 0 & 2 & 2 & 2 & 2 & 4 & 0 \\ 2 & 2 & 2 & 2 & 2 & 0 & 4\end{array}$

$4 \rightarrow \quad 108 \times \quad P(7,1)$

$$
72 \times \quad P(7,1)
$$

$108 \times \quad P(7,1)$

$$
S=\begin{array}{rrrrrrr}
0 & 0 & 0 & 1 & 1 & 0 & 1 \\
0 & 0 & 1 & 0 & 1 & 0 & 1 \\
-1 & 0 & -1 & -1 & -1 & 0 & -1 \\
0 & -1 & -1 & -1 & -1 & 0 & -1 \\
-1 & -1 & -1 & -1 & -2 & 0 & -1 \\
1 & 1 & 1 & 1 & 1 & 0 & 0 \\
1 & 1 & 1 & 1 & 1 & 1 & 1
\end{array}
$$$$
108 \times \quad P(7,1)
$$$$
\begin{array}{rrrrrrrrrrrrrrrr}
4 & 0 & 2 & 2 & 2 & 2 & 2 & & 0 & -1 & -1 & 0 & 0 & 0 & 0 \\
\text { Voisine }= & 0 & 4 & 2 & 2 & 2 & 6 & 0 & & 0 & -1 & -2 & -1 & -1 & -1 & -2 \\
2 & 2 & 4 & 2 & 2 & 4 & 2 & -1 & 0 & 0 & -1 & 0 & 0 & 0 \\
2 & 2 & 2 & 4 & 2 & 4 & 2 & S=-1 & 0 & 0 & 0 & -1 & 0 & 0 \\
2 & 2 & 2 & 2 & 4 & 4 & 2 & -1 & 0 & 0 & 0 & 0 & -1 & 0 \\
2 & 6 & 4 & 4 & 4 & 12 & 0 & 1 & 1 & 1 & 1 & 1 & 1 & 1 \\
2 & 0 & 2 & 2 & 2 & 0 & 4 & 2 & 1 & 1 & 1 & 1 & 1 & 0
\end{array}
$$ 
$5 \rightarrow \quad 324 \times \quad P(7,1)$

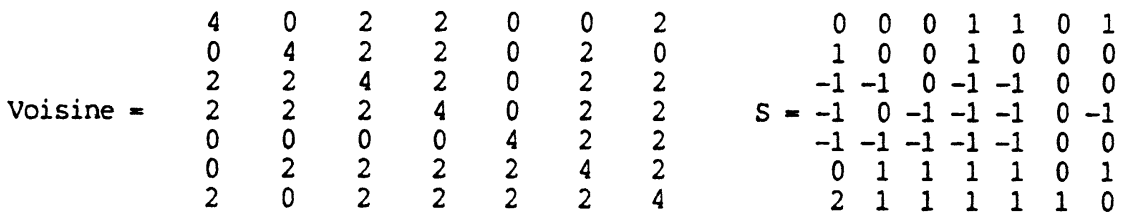

$6 \rightarrow \quad 324 \times \quad P(7,1)$

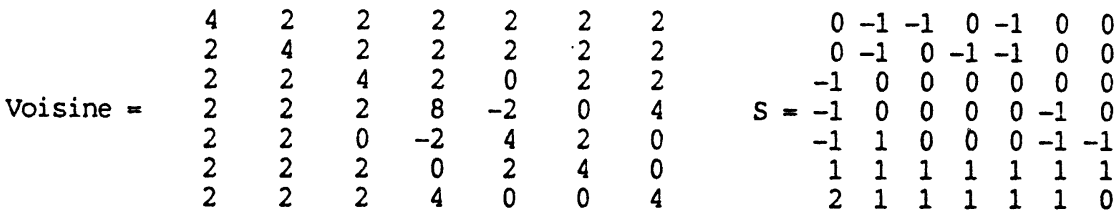

$7 \rightarrow \quad 18 \times \quad P(7,3)$

voisine = $\begin{array}{rrrrrrrrrrrrrr}4 & 1 & 2 & 2 & 2 & 3 & 2 & 0 & 0 & -1 & 0 & 1 & 0 & 0 \\ 1 & 4 & 2 & 2 & 2 & 3 & 2 & 0 & 0 & 0 & -1 & 1 & 0 & 0 \\ 2 & 2 & 4 & 1 & 1 & 3 & 2 & -1 & 0 & 0 & 0 & -1 & -1 & 0 \\ 2 & 2 & 1 & 4 & 1 & 3 & 2 & S=-1 & 0 & 0 & 0 & -1 & 0 & -1 \\ -1 & 0 & 0 & 0 & -1 & 0 & 0 \\ 2 & 2 & 1 & 1 & 4 & 3 & 2 & 1 & 0 & 0 & 0 & 0 & 0 & 0 \\ 3 & 3 & 3 & 3 & 3 & 6 & 3 & 1 & 1 & 1 & 1 & 1 & 1 & 1\end{array}$

$8 \rightarrow \quad 27 \times \quad P(7,3)$

Voisine $=\begin{array}{rrrrrrrrrrrrrr}4 & 1 & 2 & 2 & 3 & 3 & 2 & 0 & 0 & -1 & 0 & 0 & 0 & 0 \\ 1 & 4 & 2 & 2 & 3 & 3 & 2 & 0 & 0 & -1 & 0 & 0 & 0 & -1 \\ 2 & 2 & 4 & 1 & 2 & 3 & 2 & -1 & 0 & 0 & 0 & -1 & -1 & 0 \\ 2 & 2 & 1 & 4 & 2 & 3 & 2 & -1 & 0 & 0 & -1 & 0 & -1 & 0 \\ 3 & 3 & 2 & 2 & 6 & 4 & 3 & -1 & 0 & 0 & 0 & 0 & 0 & 0 \\ 3 & 3 & 3 & 3 & 4 & 6 & 2 & 1 & 0 & 1 & 0 & 0 & 1 & 0 \\ 2 & 2 & 2 & 2 & 3 & 2 & 4 & 1 & -1 & 0 & 0 & 0 & 0 & 0\end{array}$

$9 \rightarrow \quad 162 \times \quad \mathrm{P}(7,3)$

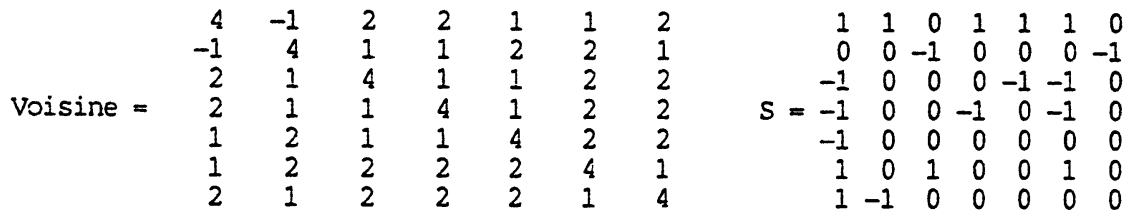

$10 \rightarrow \quad 54 \mathrm{X} \cdot \mathrm{P}(7,4)$

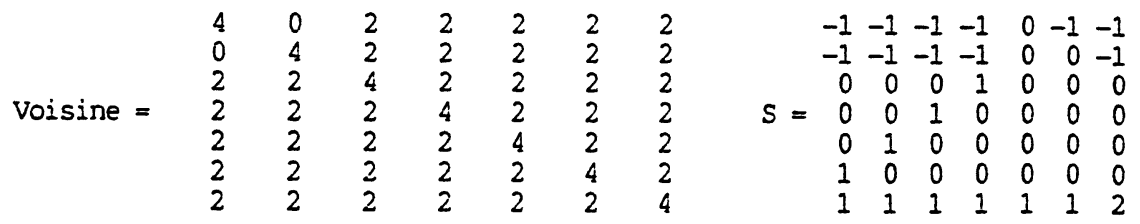


$11 \rightarrow$ Voisine $=\begin{array}{rrrrrrr}4 & 2 / 3 & 2 & 2 & 2 & 8 / 3 & 2 \\ 2 / 3 & 4 & 2 & 2 & 2 & 8 / 3 & 4 / 3 \\ 2 & 2 & 4 & 4 / 3 & 4 / 3 & 8 / 3 & 2 \\ 2 & 2 & 4 / 3 & 4 & 4 / 3 & 8 / 3 & 2 \\ 2 & 2 & 4 / 3 & 4 / 3 & 4 & 8 / 3 & 2 \\ 8 / 3 & 8 / 3 & 8 / 3 & 8 / 3 & 8 / 3 & 16 / 3 & 4 / 3 \\ 2 & 4 / 3 & 2 & 2 & 2 & 4 / 3 & 4\end{array}$

$12 \rightarrow$

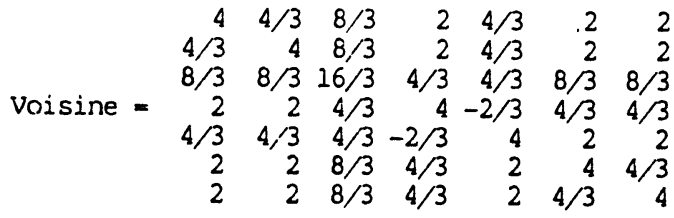

$13 \rightarrow$

$216 \times \quad P(7,6)$

$\begin{array}{rrrrrrr}4 & 2 / 3 & 8 / 3 & 4 / 3 & 2 & 2 & 2 \\ 2 / 3 & 4 & 10 / 3 & 2 & 2 / 3 & 2 & 2\end{array}$

$\begin{array}{lllllll}8 / 3 & 10 / 3 & 20 / 3 & 8 / 3 & 2 / 3 & 10 / 3 & 10 / 3\end{array}$

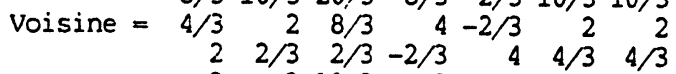
$\begin{array}{rrrrrrr}2 & 2 / 3 & 2 / 3 & -2 / 3 & 4 & 4 / 3 & 4 / 3 \\ 2 & 2 & 10 / 3 & 2 & 4 / 3 & 4 & 4 / 3 \\ 2 & 2 & 10 / 3 & 2 & 4 / 3 & 4 / 3 & 4\end{array}$

$14 \rightarrow \quad 108 \times \quad P(7,7)$

Voisine = $\begin{array}{lllllll}4 & 1 & 2 & 2 & 2 & 2 & 2 \\ 1 & 4 & 2 & 2 & 1 & 1 & 2 \\ 2 & 2 & 4 & 1 & 0 & 1 & 2 \\ 2 & 2 & 1 & 4 & 1 & 2 & 2 \\ 2 & 1 & 0 & 1 & 4 & 2 & 2 \\ 2 & 1 & 1 & 2 & 2 & 4 & 1 \\ 2 & 2 & 2 & 2 & 2 & 1 & 4\end{array}$

$15 \rightarrow \quad 324 \times \quad P(7,7)$

Voisine = $\begin{array}{lllllll}4 & 0 & 2 & 1 & 2 & 2 & 1 \\ 0 & 4 & 1 & 2 & 2 & 1 & 2 \\ 2 & 1 & 4 & 1 & 1 & 2 & 2 \\ 1 & 2 & 1 & 4 & 1 & 2 & 2 \\ 2 & 2 & 1 & 1 & 4 & 2 & 2 \\ 2 & 1 & 2 & 2 & 2 & 4 & 1 \\ 1 & 2 & 2 & 2 & 2 & 1 & 4\end{array}$

$16 \rightarrow$

Voisine = $\begin{array}{lllllll}4 & 0 & 2 & 1 & 2 & 2 & 2 \\ 0 & 4 & 1 & 2 & 3 & 2 & 2 \\ 2 & 1 & 4 & 0 & 1 & 2 & 2 \\ 1 & 2 & 0 & 4 & 2 & 2 & 2 \\ 2 & 3 & 1 & 2 & 6 & 3 & 3 \\ 2 & 2 & 2 & 2 & 3 & 4 & 2 \\ 2 & 2 & 2 & 2 & 3 & 2 & 4\end{array}$

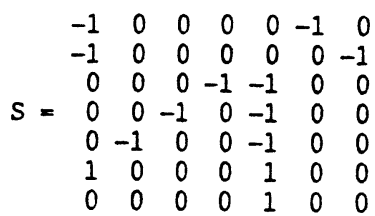

$\begin{array}{lllllll}0 & 0 & -1 & 0 & 0 & 0 & 0\end{array}$

$\begin{array}{lllllll}0 & 0 & 0 & -1 & 0 & 0 & 0\end{array}$

$\begin{array}{rrrrrrr}0 & 0 & 0 & 0 & -1 & 0 & 0\end{array}$

$S=\begin{array}{rrrrrrr}0 & 0 & 1 & 1 & 0 & 0 & 1 \\ -1 & -1 & 0 & 0 & -1 & -1 & 0\end{array}$

$\begin{array}{lllllll}0 & 1 & 0 & 0 & 1 & 0 & 0 \\ 1 & 0 & 0 & 0 & 1 & 0 & 0\end{array}$

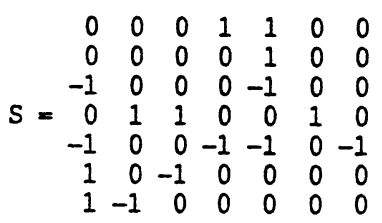

$S=\begin{array}{rrrrrrr}0 & 0 & 0 & 0 & 0 & 1 & 0 \\ 0 & 0 & -1 & 0 & 0 & 1 & 0 \\ -1 & -1 & 0 & 0 & 0 & -1 & 0 \\ -1 & 0 & 0 & 0 & 0 & -1 & 0 \\ -1 & 0 & 0 & 0 & -1 & -1 & 0 \\ 1 & 0 & 0 & -1 & 0 & 0 & 0 \\ 1 & 0 & 0 & 0 & 0 & 0 & -1\end{array}$

$S=\begin{array}{rrrrrrr}0 & 0 & 0 & 1 & 1 & 1 & 0 \\ -1 & -1 & -1 & 0 & 0 & 0 & -1 \\ 0 & 0 & 0 & -1 & 0 & 0 & 1 \\ 0 & 1 & 0 & 0 & 0 & 0 & 1 \\ 0 & 0 & 0 & 0 & 0 & 0 & 1 \\ 0 & 0 & 0 & 0 & -1 & 0 & -1 \\ 1 & 0 & 0 & 0 & 0 & 0 & -1\end{array}$

$\begin{array}{rrrrrrr}0 & 0 & 0 & -1 & 0 & -1 & 0 \\ 0 & 0 & -1 & -1 & 0 & 0 & 0\end{array}$

$\begin{array}{rrrrrrr}-1 & 0 & 0 & 0 & -1 & 0 & 0 \\ -1 & -1 & 0 & 0 & 0 & 0 & 0\end{array}$

$\begin{array}{ccccccc}-1 & 0 & 0 & 1 & 0 & 0 & 0\end{array}$

$\begin{array}{rrrrrrr}1 & 0 & 0 & 0 & 0 & 0 & 0 \\ 1 & 0 & 0 & 0 & 0 & 0 & -1\end{array}$ 
$17 \rightarrow$ Voisine = $\begin{array}{lllllll}4 & 0 & 1 & 1 & 2 & 2 & 1 \\ 0 & 4 & 2 & 2 & 2 & 2 & 2 \\ 1 & 2 & 4 & 1 & 1 & 2 & 2 \\ 1 & 2 & 1 & 4 & 1 & 2 & 2 \\ 2 & 2 & 1 & 1 & 4 & 2 & 2 \\ 2 & 2 & 2 & 2 & 2 & 4 & 1 \\ 1 & 2 & 2 & 2 & 2 & 1 & 4\end{array}$ $18 \rightarrow$ $\begin{array}{llllllll}4 & 1 & 2 & 2 & 2 & 3 & 1 \\ \text { Voisine }= & 4 & 2 & 2 & 2 & 3 & 1 \\ 2 & 2 & 4 & 2 & 1 & 3 & 2 \\ 2 & 2 & 2 & 4 & 1 & 3 & 2 \\ 2 & 2 & 1 & 1 & 4 & 3 & 1 \\ 3 & 3 & 3 & 3 & 3 & 6 & 1 \\ 1 & 1 & 2 & 2 & 1 & 1 & 4\end{array}$ $19 \rightarrow$ $\begin{array}{rrrrrrr}4 & 4 / 5 & 2 & 2 & 2 & 6 / 5 & 2 \\ 4 / 5 & 4 & 2 & 2 & 2 & 6 / 5 & 2 \\ 2 & 2 & 4 & 6 / 5 & 6 / 5 & 8 / 5 & 2 \\ \text { voisine }= & 2 & 6 / 5 & 4 & 6 / 5 & 8 / 5 & 2 \\ 2 & 2 & 6 / 5 & 6 / 5 & 4 & 8 / 5 & 2 \\ 6 / 5 & 6 / 5 & 8 / 5 & 8 / 5 & 8 / 5 & 4 & 0 \\ 2 & 2 & 2 & 2 & 2 & 0 & 4\end{array}$ $20 \rightarrow \quad 648 \times \quad P(7,17)$ Voisine $=\begin{array}{rrrrrrr}4 & 0 & 4 / 3 & 2 & 4 / 3 & 2 & 2 \\ 0 & 4 & 2 & 4 / 3 & 2 & 2 & 4 / 3 \\ 4 / 3 & 2 & 4 & 2 / 3 & 4 / 3 & 2 & 2 \\ 2 & 4 / 3 & 2 / 3 & 4 & 2 / 3 & 2 & 2 \\ 4 / 3 & 2 & 4 / 3 & 2 / 3 & 4 & 2 & 2 \\ 2 & 2 & 2 & 2 & 2 & 4 & 4 / 3 \\ 2 & 4 / 3 & 2 & 2 & 2 & 4 / 3 & 4\end{array}$ $21 \rightarrow$ Voisine = $\begin{array}{lllllll}4 & 1 & 1 & 2 & 2 & 1 & 2 \\ 1 & 4 & 2 & 2 & 2 & 2 & 2 \\ 1 & 2 & 4 & 1 & 1 & 2 & 2 \\ 2 & 2 & 1 & 4 & 1 & 2 & 2 \\ 2 & 2 & 1 & 1 & 4 & 2 & 2 \\ 1 & 2 & 2 & 2 & 2 & 4 & 1 \\ 2 & 2 & 2 & 2 & 2 & 1 & 4\end{array}$ $22 \rightarrow$ $9 \times \quad P(7,26)$

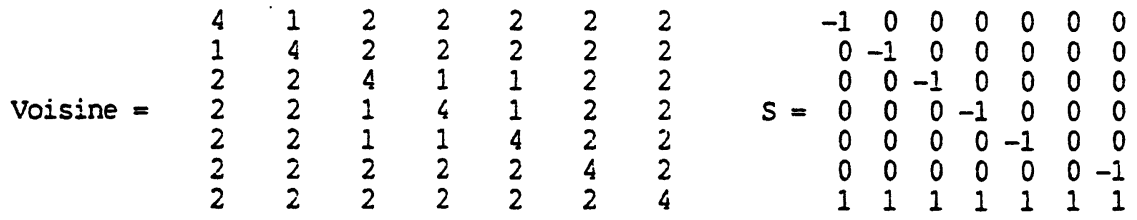

$\begin{array}{rrrrrrr}-1 & 0 & 0 & 0 & 0 & -1 & 0 \\ -1 & 0 & 0 & -1 & -1 & -1 & -1\end{array}$ $\begin{array}{lllllll}0 & 0 & -1 & 0 & 1 & 0 & 0\end{array}$ $S=\begin{array}{rrrrrrr}0 & -1 & 0 & 0 & 1 & 0 & 0\end{array}$ $\begin{array}{lllllll}0 & 0 & 0 & 0 & 1 & 1 & 0\end{array}$ $\begin{array}{lllllll}1 & 0 & 0 & 0 & -1 & 1 & 0\end{array}$ $\begin{array}{lllllll}1 & 0 & 0 & 1 & -1 & 0 & 0\end{array}$ $\begin{array}{rrrrrrr}0 & -1 & 0 & 0 & 1 & 0 & 0 \\ 0 & 0 & -1 & 0 & 1 & 0 & 0\end{array}$ $\begin{array}{lllllll}-1 & 0 & 0 & -1 & -1 & -1 & -1\end{array}$ $\begin{array}{rrrrrrr}1 & 1 & 1 & 0 & 0 & -1 & 1 \\ 0 & 0 & 0 & 0 & -1 & -1 & 0\end{array}$ $\begin{array}{lllllll}1 & 0 & 0 & 1 & 0 & 1 & 0 \\ 1 & 0 & 0 & 0 & 0 & 1 & 0\end{array}$

$$
S=\begin{array}{rrrrrrr}
-1 & 0 & 0 & 0 & 0 & 0 & -1 \\
0 & 0 & 1 & 0 & 0 & 0 & 0 \\
0 & 0 & -1 & -1 & -1 & 0 & 0 \\
0 & -1 & -1 & -1 & -1 & -1 & 0 \\
0 & 0 & -1 & -1 & 0 & -1 & 0 \\
0 & 0 & 1 & 1 & 1 & 1 & 0 \\
1 & 0 & 1 & 1 & 1 & 1 & 0
\end{array}
$$

$162 \times P(7,22)$ 
$23 \rightarrow$

$81 \times \quad P(7,29)$

Voisine = $\begin{array}{lllllll}4 & 1 & 2 & 2 & 2 & 2 & 2 \\ 1 & 4 & 2 & 2 & 2 & 2 & 2 \\ 2 & 2 & 4 & 2 & 1 & 3 & 2 \\ 2 & 2 & 2 & 4 & 1 & 3 & 2 \\ 2 & 2 & 1 & 1 & 4 & 2 & 2 \\ 2 & 2 & 3 & 3 & 2 & 6 & 1 \\ 2 & 2 & 2 & 2 & 2 & 1 & 4\end{array}$

Nombre total de voisines : 3906

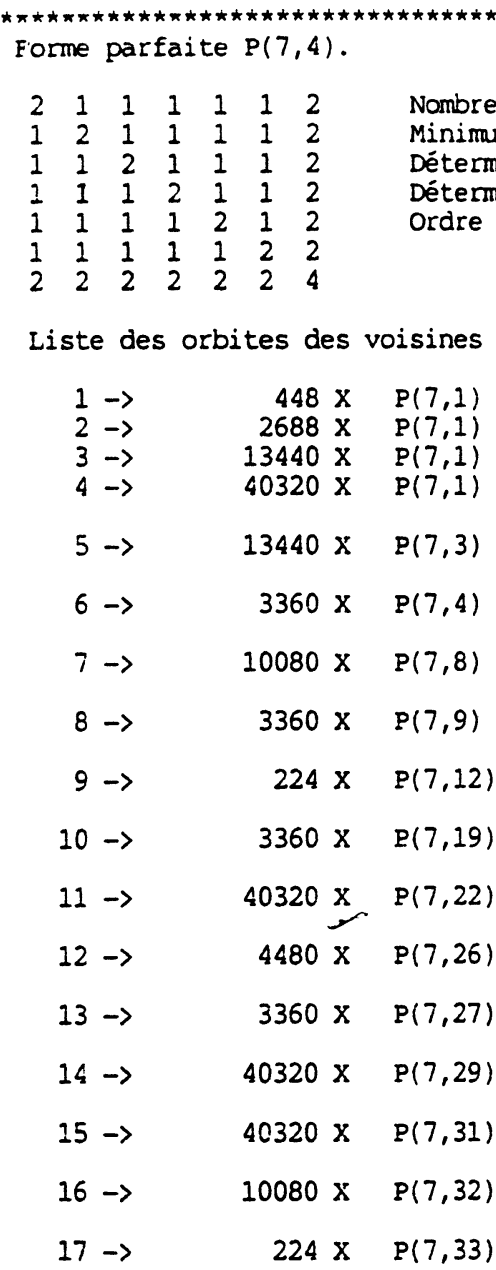

Nombre total de voisines : 229824 


\section{Forme parfaite $P(7,5)$.}

$\begin{array}{lllllllll}4 & 1 & 2 & 1 & 2 & 2 & 2 & \text { Nombre de vecteurs minimaux } & : 36 \\ 1 & 4 & 1 & 2 & 2 & 2 & 2 & \text { Minimm } & : 4 \\ 2 & 1 & 4 & 1 & 2 & 2 & 2 & \text { Déterminant } & : 512 \\ 1 & 2 & 1 & 4 & 2 & 2 & 2 & \text { Déterminant (minimum normalisé à 2) } & : 4 \\ 2 & 2 & 2 & 2 & 4 & 1 & 2 & \text { Ordre du groupe des automorphismes } & : 256 \\ 2 & 2 & 2 & 2 & 1 & 4 & 1 & & \end{array}$

$\begin{array}{lllllll}2 & 2 & 2 & 2 & 1 & 4 & 1 \\ 2 & 2 & 2 & 2 & 2 & 1 & 4\end{array}$

Liste des orbites des voisines de la forme $P(7,5)$ :

$1 \rightarrow$

$\begin{array}{lllllll}8 & 2 & 4 & 2 & 4 & 4 & 4 \\ 2 & 4 & 0 & 2 & 2 & 2 & 2 \\ 4 & 0 & 4 & 0 & 2 & 2 & 2 \\ 2 & 2 & 0 & 4 & 2 & 2 & 2 \\ 4 & 2 & 2 & 2 & 4 & 2 & 2 \\ 4 & 2 & 2 & 2 & 2 & 4 & 2 \\ 4 & 2 & 2 & 2 & 2 & 2 & 4\end{array}$

$2 \rightarrow$

Voisine $=\begin{array}{llll}4 & 0 & 2 & 0 \\ 0 & 4 & 0 & 2 \\ 2 & 0 & 4 & 0 \\ 0 & 2 & 0 & 4 \\ 2 & 0 & 2 & 0 \\ 2 & 2 & 2 & 2 \\ 2 & 2 & 2 & 2\end{array}$

$3 \rightarrow$

$16 \times \quad P(7,1)$

voisine $=\begin{array}{lllllll}4 & 0 & 0 & 0 & 2 & 2 & 2 \\ 0 & 4 & 2 & 2 & 2 & 2 & 2 \\ 0 & 2 & 4 & 2 & 2 & 2 & 2 \\ 0 & 2 & 2 & 4 & 2 & 2 & 2 \\ 2 & 2 & 2 & 2 & 4 & 2 & 2 \\ 2 & 2 & 2 & 2 & 2 & 4 & 2 \\ 2 & 2 & 2 & 2 & 2 & 2 & 4\end{array}$

$4 \rightarrow \quad 16 \times \quad P(7,1)$

Voisine $=\begin{array}{lllllll}4 & 0 & 2 & 0 & 2 & 2 & 2 \\ 0 & 4 & 0 & 2 & 2 & 0 & 2 \\ 2 & 0 & 4 & 0 & 2 & 2 & 2 \\ 0 & 2 & 0 & 4 & 2 & 0 & 2 \\ 2 & 2 & 2 & 2 & 4 & 0 & 2 \\ 2 & 0 & 2 & 0 & 0 & 4 & 2 \\ 2 & 2 & 2 & 2 & 2 & 2 & 4\end{array}$

$5 \rightarrow \quad 32 \times \quad P(7,1)$

Voisine $=\quad \begin{array}{lllllll}4 & 0 & 2 & 0 & 2 & 2 & 2 \\ 0 & 4 & 2 & 2 & 2 & 2 & 2 \\ 2 & 2 & 4 & 0 & 2 & 2 & 2 \\ 0 & 2 & 0 & 4 & 2 & 2 & 2 \\ 2 & 2 & 2 & 2 & 4 & 2 & 2 \\ 2 & 2 & 2 & 2 & 2 & 4 & 2 \\ 2 & 2 & 2 & 2 & 2 & 2 & 4\end{array}$

$$
S=\begin{array}{rrrrrrr}
-1 & -1 & -1 & -1 & -1 & -1 & -1 \\
-1 & -1 & -1 & -1 & 0 & -1 & 0 \\
-1 & -1 & -1 & 0 & 0 & 0 & 1 \\
-1 & -1 & -1 & 0 & -1 & -1 & 0 \\
1 & 1 & 2 & 1 & 1 & 1 & 1 \\
1 & 2 & 1 & 1 & 1 & 1 & 0 \\
2 & 1 & 1 & 1 & 1 & 1 & 0
\end{array}
$$

$$
\begin{array}{lll}
2 & 2 & 2 \\
0 & 2 & 2 \\
2 & 2 & 2 \\
0 & 2 & 2 \\
4 & 0 & 2 \\
0 & 4 & 2 \\
2 & 2 & 4
\end{array}
$$

$\begin{array}{lllllll}-1 & -1 & -2 & -1 & -1 & -1 & -1\end{array}$

$\begin{array}{lllllll}-1 & -1 & -1 & -1 & -1 & 0 & 0\end{array}$

$\begin{array}{lllllll}-1 & -1 & -1 & -2 & -1 & -1 & -1\end{array}$

$S=-1-1 \quad-1 \quad-1 \quad 0 \quad-1 \quad 0$

$\begin{array}{lllllll}0 & 1 & 1 & 1 & 1 & 1 & 1\end{array}$

$\begin{array}{lllllll}1 & 2 & 2 & 2 & 1 & 1 & 1 \\ 2 & 1 & 1 & 1 & 1 & 1 & 0\end{array}$

$$
S=\begin{array}{rrrrrrr}
-2 & -2 & -2 & -1 & -1 & -1 & 0 \\
-1 & -1 & -1 & -1 & -1 & 0 & 0 \\
-1 & -1 & -1 & -1 & 0 & -1 & 0 \\
-1 & -1 & -1 & 0 & -1 & -1 & 0 \\
1 & 1 & 2 & 1 & 1 & 1 & 1 \\
1 & 2 & 1 & 1 & 1 & 1 & 0 \\
2 & 1 & 1 & 1 & 1 & 1 & 0
\end{array}
$$

$$
S=\begin{array}{rrrrrrr}
-1 & -1 & -2 & -1 & -1 & -1 & -1 \\
-1 & -1 & -1 & -1 & -1 & 0 & 0 \\
-1 & -1 & -1 & -2 & -1 & -1 & -1 \\
-1 & -1 & -1 & -1 & 0 & -1 & 0 \\
1 & 2 & 2 & 2 & 1 & 1 & 1 \\
0 & 1 & 1 & 1 & 1 & 1 & 1 \\
2 & 1 & 1 & 1 & 1 & 1 & 0
\end{array}
$$

$$
\mathbf{S}=\begin{array}{rrrrrrr}
-1 & -2 & -1 & -1 & -1 & -1 & 0 \\
-1 & -1 & 0 & -1 & -1 & -1 & 0 \\
0 & 0 & -1 & -1 & 0 & 0 & -1 \\
-1 & -1 & -1 & -1 & 0 & 0 & 0 \\
1 & 1 & 1 & 1 & 0 & 1 & 0 \\
1 & 1 & 1 & 1 & 1 & 0 & 0
\end{array}
$$


$6 \rightarrow$

voisine $=\begin{array}{lllllll}4 & 0 & 0 & 2 & 2 & 2 & 2 \\ 0 & 4 & 2 & 2 & 2 & 2 & 2 \\ 0 & 2 & 4 & 0 & 2 & 2 & 2 \\ 2 & 2 & 0 & 4 & 2 & 2 & 2 \\ 2 & 2 & 2 & 2 & 4 & 2 & 2 \\ 2 & 2 & 2 & 2 & 2 & 4 & 2 \\ 2 & 2 & 2 & 2 & 2 & 2 & 4\end{array}$

$7 \rightarrow$

Voisine = $\begin{array}{rrrrrrr}4 & 0 & 2 & -2 & 0 & 2 & 2 \\ 0 & 4 & 2 & 2 & 2 & 2 & 2 \\ 2 & 2 & 4 & 0 & 2 & 2 & 2 \\ -2 & 2 & 0 & 4 & 2 & 0 & 0 \\ 0 & 2 & 2 & 2 & 4 & 0 & 0 \\ 2 & 2 & 2 & 0 & 0 & 4 & 2 \\ 2 & 2 & 2 & 0 & 0 & 2 & 4\end{array}$

$8 \rightarrow \quad 64 \times \quad P(7,1)$

voisine = $\begin{array}{lllllll}4 & 0 & 0 & 0 & 2 & 0 & 2 \\ 0 & 4 & 2 & 2 & 2 & 2 & 2 \\ 0 & 2 & 4 & 0 & 0 & 2 & 2 \\ 0 & 2 & 0 & 4 & 2 & 2 & 0 \\ 2 & 2 & 0 & 2 & 4 & 0 & 2 \\ 0 & 2 & 2 & 2 & 0 & 4 & 0 \\ 2 & 2 & 2 & 0 & 2 & 0 & 4\end{array}$

$9 \rightarrow \quad 64 \times \quad P(7,1)$

Voisine $=\begin{array}{rrrrrrr}4 & 0 & 2 & 0 & 2 & 2 & 2 \\ 0 & 4 & 0 & 2 & 2 & 0 & 2 \\ 2 & 0 & 4 & -2 & 0 & 2 & 2 \\ 0 & 2 & -2 & 4 & 2 & 0 & 0 \\ 2 & 2 & 0 & 2 & 4 & 0 & 2 \\ 2 & 0 & 2 & 0 & 0 & 4 & 0 \\ 2 & 2 & 2 & 0 & 2 & 0 & 4\end{array}$

$10 \rightarrow \quad 64 \times \quad P(7,1)$

Voisine $=\begin{array}{rrrrrrr}4 & -2 & 0 & 0 & 2 & 0 & 0 \\ -2 & 4 & 2 & 2 & 0 & 2 & 2 \\ 0 & 2 & 4 & 2 & 2 & 2 & 2 \\ 0 & 2 & 2 & 4 & 2 & 2 & 2 \\ 2 & 0 & 2 & 2 & 4 & 0 & 2 \\ 0 & 2 & 2 & 2 & 0 & 4 & 0 \\ 0 & 2 & 2 & 2 & 2 & 0 & 4\end{array}$

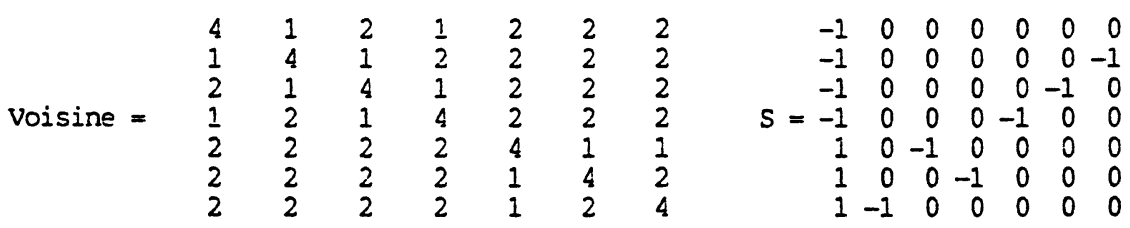

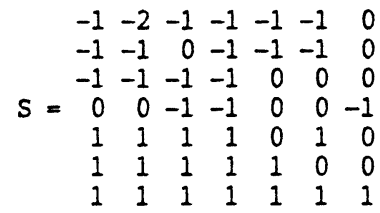$$
S=\begin{array}{rrrrrrr}
-1 & -1 & -1 & 0 & -1 & 0 & 0 \\
-1 & -1 & -2 & -1 & -1 & -1 & -1 \\
-1 & 0 & 0 & -1 & 0 & 0 & 0 \\
-1 & 0 & 0 & 0 & -1 & 0 & 0 \\
2 & 1 & 1 & 1 & 1 & 1 & 0 \\
1 & 1 & 1 & 1 & 1 & 0 & 0 \\
1 & 1 & 1 & 1 & 1 & 1 & 1
\end{array}
$$

$\begin{array}{rrrrrrr}-1 & -1 & -1 & -1 & -1 & 0 & 0 \\ -1 & -1 & -1 & -2 & -1 & -1 & -1\end{array}$

$\begin{array}{ccccccc}-1 & -1 & 0 & 0 & 0 & 0 & 1\end{array}$

$s=\begin{array}{rrrrrrr}0 & -1 & 0 & 0 & -1 & 0 & 0\end{array}$

$\begin{array}{lllllll}0 & 1 & 1 & 1 & 1 & 0 & 1 \\ 1 & 2 & 1 & 1 & 1 & 1 & 0\end{array}$

$\begin{array}{lllllll}1 & 2 & 1 & 1 & 1 & 1 & 0\end{array}$

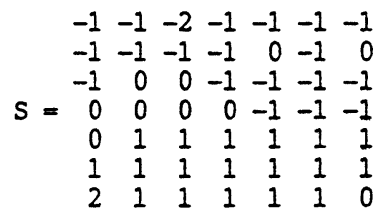

$\begin{array}{rrrrrrr}-1 & -1 & -1 & 0 & -1 & -1 & 0 \\ -1 & -1 & -1 & 0 & 0 & -1 & 0\end{array}$

$\begin{array}{lllllll}-1 & -1 & 0 & -1 & -1 & -1 & 0\end{array}$

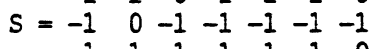

$\begin{array}{lllllll}1 & 1 & 1 & 1 & 1 & 1 & 0 \\ 2 & 1 & 1 & 1 & 1 & 1 & 0\end{array}$

$\begin{array}{lllllll}1 & 1 & 1 & 1 & 1 & 1 & 1\end{array}$
$11 \rightarrow$

$$
4 \times \quad P(7,5)
$$


$12 \rightarrow$ Voisine $=\begin{array}{lllllll}4 & 1 & 2 & 0 & 2 & 2 & 2 \\ 1 & 4 & 1 & 2 & 2 & 2 & 2 \\ 2 & 1 & 4 & 0 & 2 & 2 & 2 \\ 0 & 2 & 0 & 4 & 1 & 2 & 1 \\ 2 & 2 & 2 & 1 & 4 & 1 & 2 \\ 2 & 2 & 2 & 2 & 1 & 4 & 1 \\ 2 & 2 & 2 & 1 & 2 & 1 & 4\end{array}$

$13 \rightarrow$

$64 \times \quad P(7,5)$

$$
S=\begin{array}{rrrrrrr}
-1 & 1 & 0 & 0 & 0 & 0 & 0 \\
-1 & 0 & -1 & 0 & -1 & 0 & -1 \\
-1 & 0 & 0 & 1 & 0 & 0 & 0 \\
-1 & 0 & 0 & 0 & 0 & 0 & 0 \\
1 & 0 & 0 & 0 & 0 & 0 & 1 \\
1 & -1 & 0 & -1 & 0 & -1 & 0 \\
1 & 0 & 0 & 0 & 1 & 0 & 0
\end{array}
$$

$14 \rightarrow$

$$
\begin{array}{rrrrrrrrrrrrrrr}
4 & 0 & 2 & 0 & 2 & 1 & 2 & -1 & 0 & 0 & 1 & 0 & 0 & 0 \\
\text { Voisine = } & 0 & 4 & 1 & 2 & 2 & 2 & 2 & -1 & 0 & 0 & 0 & 0 & -1 & 0 \\
2 & 1 & 4 & 1 & 2 & 2 & 2 & & -1 & -1 & -1 & -1 & -1 & -1 & -1 \\
0 & 2 & 1 & 4 & 2 & 2 & 2 & S=-1 & 0 & 0 & 0 & 0 & 0 & 0 \\
2 & 2 & 2 & 2 & 4 & 1 & 2 & 1 & 1 & 0 & 0 & 0 & 1 & 0 \\
1 & 2 & 2 & 2 & 1 & 4 & 2 & & 1 & 0 & 1 & 0 & 0 & 1 & 0 \\
2 & 2 & 2 & 2 & 2 & 2 & 4 & & 1 & 0 & 0 & 0 & 1 & 0 & 0
\end{array}
$$

$15 \rightarrow \quad 64 \times \quad P(7,5)$

$$
\begin{array}{rrrrrrrrrrrrrrrr}
4 & 0 & 1 & 1 & 1 & 2 & 1 & -1 & 0 & 0 & 0 & -1 & 0 & 0 \\
0 & 4 & 1 & 1 & 2 & 2 & 2 & -1 & 1 & 0 & 1 & 0 & 0 & 1 \\
1 & 1 & 4 & 2 & 2 & 2 & 2 & -1 & 0 & 0 & 0 & 0 & -1 & 0 \\
1 & 1 & 2 & 4 & 2 & 2 & 2 & S=-1 & 0 & 0 & 0 & 0 & 0 & 0 \\
1 & 2 & 2 & 2 & 4 & 1 & 2 & 1 & 0 & 0 & -1 & 0 & 0 & 0 \\
2 & 2 & 2 & 2 & 1 & 4 & 1 & 2 & 0 & 1 & 0 & 1 & 1 & 0 \\
1 & 2 & 2 & 2 & 2 & 1 & 4 & 1 & -1 & 0 & 0 & 0 & 0 & 0
\end{array}
$$

$16 \rightarrow \quad 64 \times \quad P(7,5)$

$$
\text { Voisine = } \begin{array}{rrrrrrrrrrrrrrrr}
4 & 0 & 2 & 0 & 2 & 2 & 1 & -1 & 0 & 0 & 0 & -1 & 0 & 0 \\
0 & 4 & 1 & 2 & 2 & 2 & 2 & & -1 & 0 & -1 & 0 & -1 & 0 & -1 \\
2 & 1 & 4 & 0 & 2 & 2 & 2 & -1 & 0 & -1 & 0 & 0 & -1 & 0 \\
0 & 2 & 0 & 4 & 2 & 2 & 1 & S=-1 & 0 & 0 & 0 & 0 & -1 & 0 \\
2 & 2 & 2 & 2 & 4 & 2 & 2 & & 1 & 0 & 0 & -1 & 0 & 0 & 0 \\
2 & 2 & 2 & 2 & 2 & 4 & 1 & 1 & 0 & 1 & 0 & 1 & 1 & 0 \\
1 & 2 & 2 & 1 & 2 & 1 & 4 & & 1 & 1 & 1 & 1 & 1 & 1 & 1
\end{array}
$$

$17 \rightarrow \quad 4 \times \quad P(7,6)$

$$
\begin{array}{rrrrrrrrrrrrrrr}
4 & 2 / 3 & 2 & 4 / 3 & 2 & 2 & 2 & & -1 & 0 & 0 & 0 & 0 & -1 & 0 \\
2 / 3 & 4 & 4 / 3 & 2 & 2 & 2 & 2 & & -1 & 0 & 0 & 0 & 0 & 0 & -1 \\
2 & 4 / 3 & 4 & 2 / 3 & 2 & 2 & 2 & & -1 & 0 & 0 & 0 & 0 & 0 & 0 \\
\text { Voisine }= & 4 / 3 & 2 & 2 / 3 & 4 & 2 & 2 & 2 & S=-1 & 0 & 0 & 0 & -1 & 0 & 0 \\
2 & 2 & 2 & 2 & 4 & 4 / 3 & 4 / 3 & & 1 & 0 & 0 & -1 & 0 & 0 & 0 \\
2 & 2 & 2 & 2 & 4 / 3 & 4 & 4 / 3 & & 1 & 0 & -1 & 0 & 0 & 0 & 0 \\
2 & 2 & 2 & 2 & 4 / 3 & 4 / 3 & 4 & & 1 & -1 & 0 & 0 & 0 & 0 & 0
\end{array}
$$


$18 \rightarrow$

$32 \times \quad P(7,7)$

$\begin{array}{llllllll} & 4 & 0 & 2 & 1 & 2 & 2 & 2 \\ 0 & 4 & 1 & 2 & 2 & 1 & 2 \\ 2 & 1 & 4 & 1 & 2 & 2 & 2 \\ \text { Voisine }= & 1 & 2 & 1 & 4 & 2 & 2 & 2 \\ 2 & 2 & 2 & 2 & 4 & 1 & 2 \\ 2 & 1 & 2 & 2 & 1 & 4 & 1 \\ 2 & 2 & 2 & 2 & 2 & 1 & 4\end{array}$ $S=\begin{array}{rrrrrrr}-1 & 0 & 0 & -1 & -1 & -1 & 0 \\ 0 & 1 & 1 & 0 & 0 & 0 & 1 \\ -1 & 0 & 0 & 1 & 0 & 0 & 0 \\ -1 & -1 & 0 & 0 & 0 & 0 & 0 \\ 1 & 0 & 0 & 0 & 0 & 0 & 0 \\ 1 & 0 & 0 & 0 & 1 & 0 & 0 \\ 1 & 0 & 0 & 0 & 0 & 0 & -1\end{array}$

$19 \rightarrow \quad 64 \times \quad P(7,7)$

$\begin{array}{lllllll}4 & 0 & 2 & 1 & 2 & 2 & 2 \\ 0 & 4 & 1 & 2 & 1 & 2 & 2 \\ 2 & 1 & 4 & 1 & 2 & 2 & 2 \\ 1 & 2 & 1 & 4 & 2 & 2 & 2 \\ 2 & 1 & 2 & 2 & 4 & 1 & 1 \\ 2 & 2 & 2 & 2 & 1 & 4 & 2 \\ 2 & 2 & 2 & 2 & 1 & 2 & 4\end{array}$

$\mathrm{S}=\begin{array}{rrrrrrr}-1 & 0 & 0 & -1 & -1 & -1 & 0 \\ 0 & 1 & 1 & 0 & 0 & 0 & 1 \\ -1 & 0 & 0 & 1 & 0 & 0 & 0 \\ -1 & -1 & 0 & 0 & 0 & 0 & 0 \\ 1 & 0 & 0 & 0 & 1 & 0 & 0 \\ 1 & 0 & 0 & 0 & 0 & 0 & 0 \\ 1 & 0 & 0 & 0 & 0 & 0 & -1\end{array}$

$20 \rightarrow \quad 128 \times \quad P(7,7)$

$\begin{array}{lllllll}4 & 0 & 2 & 0 & 1 & 2 & 2 \\ 0 & 4 & 1 & 2 & 2 & 2 & 2 \\ 2 & 1 & 4 & 1 & 2 & 2 & 2 \\ 0 & 2 & 1 & 4 & 2 & 2 & 1 \\ 1 & 2 & 2 & 2 & 4 & 1 & 1 \\ 2 & 2 & 2 & 2 & 1 & 4 & 2 \\ 2 & 2 & 2 & 1 & 1 & 2 & 4\end{array}$

$S=\begin{array}{rrrrrrr}0 & 0 & 1 & 0 & 0 & 0 & 1 \\ 0 & 0 & 1 & 1 & 1 & 1 & 1 \\ 0 & -1 & 0 & 0 & 1 & 0 & 0 \\ 0 & 0 & 1 & 0 & 0 & -1 & 0 \\ 0 & 0 & -1 & 0 & -1 & 0 & -1 \\ 0 & 0 & -1 & -1 & -1 & 0 & -1 \\ 1 & 1 & 0 & 0 & 0 & 0 & 0\end{array}$

$21 \rightarrow$

$128 \times \quad P(7,7)$

Voisine $=\begin{array}{lllllll}4 & 1 & 2 & 0 & 2 & 2 & 1 \\ 1 & 4 & 1 & 1 & 1 & 2 & 2 \\ 2 & 1 & 4 & 0 & 2 & 2 & 2 \\ 0 & 1 & 0 & 4 & 1 & 1 & 2 \\ 2 & 1 & 2 & 1 & 4 & 0 & 2 \\ 2 & 2 & 2 & 1 & 0 & 4 & 1 \\ 1 & 2 & 2 & 2 & 2 & 1 & 4\end{array}$

$S=\begin{array}{rrrrrrr}-1 & -1 & -1 & 0 & 0 & 0 & -1 \\ -1 & 0 & 0 & 1 & 0 & 0 & 0 \\ -1 & -1 & 0 & 0 & -1 & -1 & -1 \\ -1 & -1 & 0 & 0 & 0 & 0 & 0 \\ 1 & 1 & 0 & 0 & 0 & 1 & 1 \\ 1 & 1 & 0 & -1 & 0 & 0 & 1 \\ 1 & 0 & 0 & 0 & 1 & 0 & 0\end{array}$

$22 \rightarrow \quad 128 \times \quad \mathrm{P}(7,7)$

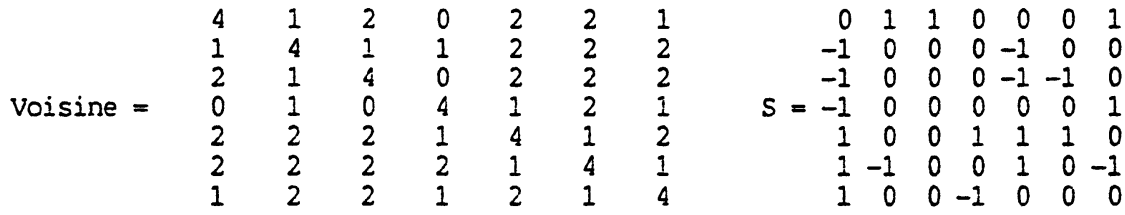

$23 \rightarrow \quad 128 \times \quad P(7,7)$

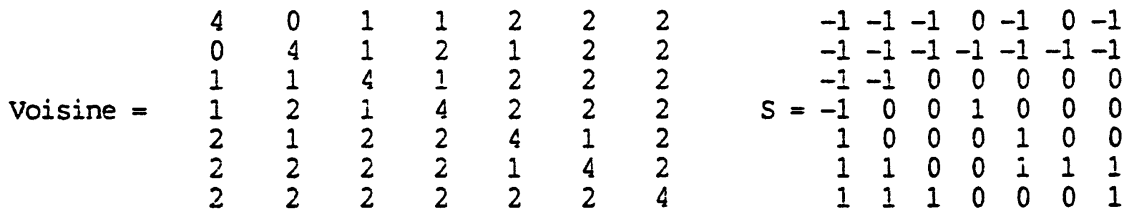


$24->$ Voisine $=\begin{array}{lllllll}4 & 1 & 1 & 1 & 2 & 2 & 2 \\ 1 & 4 & 1 & 2 & 2 & 2 & 2 \\ 1 & 1 & 4 & 0 & 1 & 2 & 2 \\ 1 & 2 & 0 & 4 & 2 & 2 & 1 \\ 2 & 2 & 1 & 2 & 4 & 1 & 2 \\ 2 & 2 & 2 & 2 & 1 & 4 & 1 \\ 2 & 2 & 2 & 1 & 2 & 1 & 4\end{array}$

$25 \rightarrow$ Voisine = $\begin{array}{lllllll}4 & 0 & 2 & 0 & 2 & 1 & 2 \\ 0 & 4 & 1 & 2 & 1 & 2 & 2 \\ 2 & 1 & 4 & 0 & 1 & 2 & 2 \\ 0 & 2 & 0 & 4 & 2 & 1 & 2 \\ 2 & 1 & 1 & 2 & 4 & 0 & 2 \\ 1 & 2 & 2 & 1 & 0 & 4 & 1 \\ 2 & 2 & 2 & 2 & 2 & 1 & 4\end{array}$

$26 \rightarrow$

$$
\mathbf{S}=\begin{array}{rrrrrrr}
0 & 1 & 0 & 0 & 0 & 0 & 1 \\
0 & 1 & 1 & 0 & 0 & 0 & 1 \\
0 & 0 & 0 & -1 & 0 & 0 & 1 \\
0 & 0 & 0 & -1 & -1 & -1 & 0 \\
-1 & -1 & -1 & 0 & 0 & 0 & -1 \\
0 & -1 & 0 & 1 & 1 & 0 & -1 \\
1 & 0 & 0 & 0 & 0 & 0 & -1
\end{array}
$$$$
S=\begin{array}{rrrrrrr}
-1 & 1 & 1 & 0 & 1 & 0 & 1 \\
-1 & 1 & 0 & -1 & 0 & 0 & 0 \\
0 & 0 & -1 & 0 & 1 & 0 & 0 \\
-1 & 0 & 0 & 0 & 1 & -1 & 0 \\
1 & 0 & 0 & 0 & -1 & 0 & 0 \\
1 & 0 & 0 & 1 & -1 & 0 & 0 \\
1 & -1 & 0 & 0 & -1 & 1 & -1
\end{array}
$$

$27 \rightarrow$

voisine = $\begin{array}{rrrrrrr}4 & 0 & 2 & 0 & 2 & 2 & 1 \\ 0 & 4 & 1 & 2 & 2 & 1 & 2 \\ 2 & 1 & 4 & 0 & 2 & 2 & 2 \\ 0 & 2 & 0 & 4 & 2 & 1 & 1 \\ 2 & 2 & 2 & 2 & 4 & 1 & 2 \\ 2 & 1 & 2 & 1 & 1 & 4 & 0 \\ 1 & 2 & 2 & 1 & 2 & 0 & 4\end{array}$

$28 \rightarrow$

Voisine = $\begin{array}{lllllll}4 & 0 & 1 & 1 & 2 & 1 & 2 \\ 0 & 4 & 1 & 2 & 1 & 2 & 2 \\ 1 & 1 & 4 & 1 & 1 & 2 & 2 \\ 1 & 2 & 1 & 4 & 2 & 2 & 2 \\ 2 & 1 & 1 & 2 & 4 & 0 & 2 \\ 1 & 2 & 2 & 2 & 0 & 4 & 1 \\ 2 & 2 & 2 & 2 & 2 & 1 & 4\end{array}$

$29 \rightarrow$

$$
\text { voisine }=\begin{array}{rrrrrrr}
4 & 0 & 2 & 2 / 3 & 4 / 3 & 2 & 2 \\
0 & 4 & 2 / 3 & 2 & 4 / 3 & 2 & 2 \\
2 & 2 / 3 & 4 & 4 / 3 & 2 & 2 & 2 \\
2 / 3 & 2 & 4 / 3 & 4 & 2 & 2 & 2 \\
4 / 3 & 4 / 3 & 2 & 2 & 4 & 2 / 3 & 4 / 3 \\
2 & 2 & 2 & 2 & 2 / 3 & 4 & 2 \\
2 & 2 & 2 & 2 & 4 / 3 & 2 & 4
\end{array}
$$

$$
S=\begin{array}{rrrrrrr}
-1 & 0 & -1 & -1 & 0 & -1 & -1 \\
0 & 0 & -1 & 0 & 0 & 0 & 0 \\
-1 & 0 & -1 & 0 & 0 & -1 & 0 \\
-1 & -1 & -1 & -1 & -1 & -1 & -1 \\
1 & 1 & 1 & 1 & 0 & 1 & 1 \\
1 & 0 & 1 & 1 & 0 & 1 & 0 \\
1 & 0 & 1 & 0 & 0 & 0 & 0
\end{array}
$$$$
S=\begin{array}{rrrrrrr}
-1 & 0 & 0 & -1 & 0 & -1 & -1 \\
-1 & 0 & -1 & -1 & 0 & -1 & -1 \\
-1 & -1 & -1 & -1 & -1 & -1 & -1 \\
0 & -1 & 0 & -1 & -1 & 0 & -1 \\
1 & 1 & 0 & 1 & 0 & 0 & 1 \\
1 & 1 & 1 & 1 & 1 & 1 & 2 \\
1 & 0 & 1 & 1 & 1 & 1 & 1
\end{array}
$$ 
$30 \rightarrow$

$$
\text { Voisine }=\begin{array}{rrrrrrr}
4 & 2 / 3 & 2 & 0 & 2 & 4 / 3 & 2 \\
2 / 3 & 4 & 4 / 3 & 2 & 2 & 2 & 2 \\
2 & 4 / 3 & 4 & 2 / 3 & 2 & 2 & 2 \\
0 & 2 & 2 / 3 & 4 & 2 & 4 / 3 & 2 \\
2 & 2 & 2 & 2 & 4 & 2 / 3 & 2 \\
4 / 3 & 2 & 2 & 4 / 3 & 2 / 3 & 4 & 4 / 3 \\
2 & 2 & 2 & 2 & 2 & 4 / 3 & 4
\end{array}
$$$$
31 \rightarrow
$$$$
\text { Voisine }=\begin{array}{rrrrrrr}
4 & 2 / 3 & 4 / 3 & 2 / 3 & 2 & 2 & 4 / 3 \\
2 / 3 & 4 & 4 / 3 & 2 & 2 & 2 & 2 \\
4 / 3 & 4 / 3 & 4 & 2 / 3 & 2 & 2 & 2 \\
2 / 3 & 2 & 2 / 3 & 4 & 2 & 2 & 4 / 3 \\
2 & 2 & 2 & 2 & 4 & 4 / 3 & 2 \\
2 & 2 & 2 & 2 & 4 / 3 & 4 & 2 / 3 \\
4 / 3 & 2 & 2 & 4 / 3 & 2 & 2 / 3 & 4
\end{array}
$$$$
32 \rightarrow
$$$$
128 \times \quad P(7,11)
$$$$
\text { Voisine }=\begin{array}{rrrrrrr}
4 & 4 / 3 & 2 & 2 / 3 & 2 & 2 & 2 \\
4 / 3 & 4 & 2 / 3 & 4 / 3 & 2 & 2 & 2 \\
2 & 2 / 3 & 4 & 0 & 4 / 3 & 2 & 2 \\
2 / 3 & 4 / 3 & 0 & 4 & 4 / 3 & 2 & 4 / 3 \\
2 & 2 & 4 / 3 & 4 / 3 & 4 & 2 / 3 & 2 \\
2 & 2 & 2 & 2 & 2 / 3 & 4 & 4 / 3 \\
2 & 2 & 2 & 4 / 3 & 2 & 4 / 3 & 4
\end{array}
$$$$
33 \rightarrow
$$

$$
32 \times \quad P(7,13)
$$$$
\text { voisine }=\begin{array}{rrrrrrr}
4 & 1 / 2 & 2 & 0 & 2 & 3 / 2 & 3 / 2 \\
1 / 2 & 4 & 3 / 2 & 2 & 2 & 2 & 2 \\
2 & 3 / 2 & 4 & 1 / 2 & 2 & 2 & 2 \\
0 & 2 & 1 / 2 & 4 & 2 & 3 / 2 & 3 / 2 \\
2 & 2 & 2 & 2 & 4 & 1 & 2 \\
3 / 2 & 2 & 2 & 3 / 2 & 1 & 4 & 1 / 2 \\
3 / 2 & 2 & 2 & 3 / 2 & 2 & 1 / 2 & 4
\end{array}
$$$$
34 \rightarrow
$$$$
32 \times \quad P(7,14)
$$$$
\text { Voisine }=\begin{array}{rrrrrrr}
4 & -2 / 3 & 2 & 2 / 3 & 2 & 4 / 3 & 4 / 3 \\
-2 / 3 & 4 & 2 / 3 & 2 & 4 / 3 & 4 / 3 & 2 \\
2 & 2 / 3 & 4 & 4 / 3 & 2 & 2 & 2 \\
2 / 3 & 2 & 4 / 3 & 4 & 2 & 2 & 2 \\
2 & 4 / 3 & 2 & 2 & 4 & 2 / 3 & 2 \\
4 / 3 & 4 / 3 & 2 & 2 & 2 / 3 & 4 & 2 / 3 \\
4 / 3 & 2 & 2 & 2 & 2 & 2 / 3 & 4
\end{array}
$$$$
35 \rightarrow
$$$$
64 \times \quad P(7,14)
$$

$$
\text { Voisine }=\begin{array}{rrrrrrr}
4 & 2 / 3 & 4 / 3 & 2 \beta & 2 & 2 & 2 / 3 \\
2 / 3 & 4 & 4 / 3 & 4 / 3 & 2 & 2 & 2 \\
4 / 3 & 4 \beta & 4 & 4 / 3 & 2 & 2 & 2 \\
2 / 3 & 4 / 3 & 4 / 3 & 4 & 2 & 2 & 2 \\
2 & 2 & 2 & 2 & 4 & 4 / 3 & 2 \\
2 & 2 & 2 & 2 & 4 / 3 & 4 & 2 / 3 \\
2 / 3 & 2 & 2 & 2 & 2 & 2 / 3 & 4
\end{array}
$$
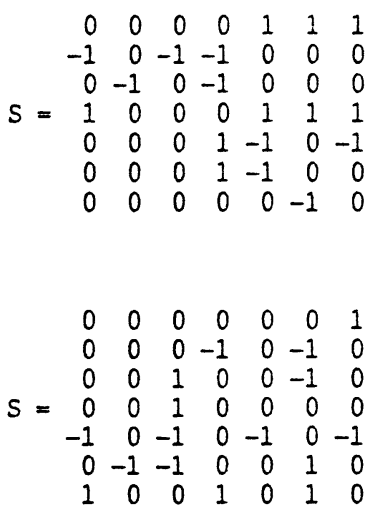

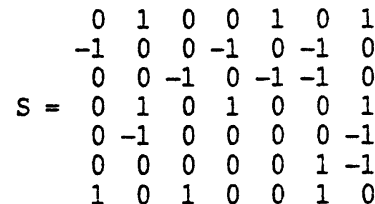

$\begin{array}{rrrrrrr}0 & 1 & 0 & 0 & 1 & 0 & 1 \\ -1 & 0 & 0 & -1 & 0 & -1 & 0 \\ 0 & 0 & -1 & 0 & -1 & -1 & 0 \\ 0 & 1 & 0 & 1 & 0 & 0 & 1 \\ 0 & -1 & 0 & 0 & 0 & 0 & -1 \\ 0 & 0 & 0 & 0 & 0 & 1 & -1 \\ 1 & 0 & 1 & 0 & 0 & 1 & 0\end{array}$

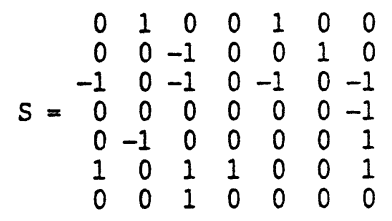

$$
S=\begin{array}{rrrrrrr}
0 & 0 & 0 & 0 & 1 & 0 & -1 \\
0 & 0 & 0 & 0 & 0 & 1 & -1 \\
-1 & 0 & 0 & 0 & -1 & 0 & -1 \\
0 & -1 & 0 & 0 & 0 & -1 & -1 \\
0 & 1 & -1 & 0 & 0 & 0 & 1 \\
0 & 0 & 0 & 0 & 0 & 0 & 1 \\
1 & 0 & 0 & -1 & 0 & 0 & 1
\end{array}
$$

$$
\mathbf{S}=\begin{array}{rrrrrrr}
-1 & 0 & 0 & 1 & 0 & -1 & 0 \\
-1 & 0 & 0 & 1 & 0 & 0 & -1 \\
0 & -1 & 1 & 1 & 0 & 0 & 0 \\
-1 & 0 & 0 & 1 & 0 & 0 & 0 \\
1 & 0 & 0 & -1 & 1 & 0 & 0 \\
1 & 1 & 0 & -1 & 0 & 1 & 1 \\
0 & 0 & 0 & -1 & -1 & 0 & 0
\end{array}
$$


$36 \rightarrow$

$$
\text { Voisine }=\begin{array}{rrrrrrr}
4 & 2 / 3 & 2 & 2 / 3 & 2 & 2 & 2 \\
2 / 3 & 4 & 4 / 3 & 2 & 2 & 2 & 2 \\
2 & 4 / 3 & 4 & 2 / 3 & 2 & 2 & 2 \\
2 / 3 & 2 & 2 / 3 & 4 & 2 & 2 & 4 / 3 \\
2 & 2 & 2 & 2 & 4 & 4 / 3 & 4 / 3 \\
2 & 2 & 2 & 2 & 4 / 3 & 4 & 4 / 3 \\
2 & 2 & 2 & 4 / 3 & 4 / 3 & 4 / 3 & 4
\end{array}
$$

$37 \rightarrow$

$32 \times \quad P(7,16)$

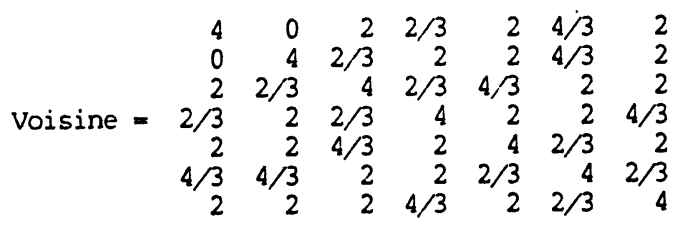

$38 \rightarrow$

$64 \times \quad P(7,17)$

voisine $=\begin{array}{rrrrrrr}4 & 2 / 3 & 2 & 2 / 3 & 2 & 2 & 4 / 3 \\ 2 / 3 & 4 & 2 / 3 & 4 / 3 & 4 / 3 & 2 & 2 \\ 2 & 2 / 3 & 4 & 2 / 3 & 2 & 2 & 2 \\ 2 / 3 & 4 / 3 & 2 / 3 & 4 & 4 / 3 & 2 & 2 \\ 2 & 4 / 3 & 2 & 4 / 3 & 4 & 2 / 3 & 2 \\ 2 & 2 & 2 & 2 & 2 / 3 & 4 & 4 / 3 \\ 4 / 3 & 2 & 2 & 2 & 2 & 4 / 3 & 4\end{array}$

$39->$

Voisine $=\begin{array}{rrrrrrr}4 & 0 & 2 & 1 / 2 & 2 & 3 / 2 & 3 / 2 \\ 0 & 4 & 1 & 2 & 2 & 3 / 2 & 2 \\ 2 & 1 & 4 & 1 & 2 & 2 & 2 \\ 1 / 2 & 2 & 1 & 4 & 2 & 2 & 3 / 2 \\ 2 & 2 & 2 & 2 & 4 & 1 & 2 \\ 3 / 2 & 3 / 2 & 2 & 2 & 1 & 4 & 1 / 2 \\ 3 / 2 & 2 & 2 & 3 / 2 & 2 & 1 / 2 & 4\end{array}$

$40 \rightarrow$

$64 \times \quad P(7,18)$

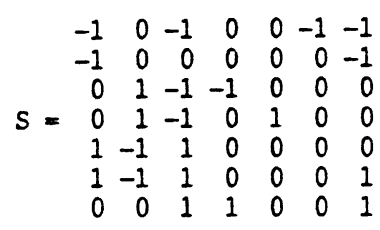

$S=\begin{array}{rrrrrrr}-1 & 0 & -1 & -1 & 0 & -1 & -1 \\ -1 & 0 & 0 & -1 & 0 & -1 & -1 \\ -1 & -1 & 0 & 0 & -1 & -1 & -1 \\ 0 & -1 & -1 & 0 & 0 & -1 & -1 \\ 1 & 0 & 1 & 0 & 0 & 1 & 1 \\ 1 & 1 & 1 & 1 & 1 & 1 & 2 \\ 1 & 1 & 0 & 1 & 0 & 1 & 1\end{array}$

$\begin{array}{rrrrrrr}-1 & -1 & 0 & 0 & 0 & 0 & -1 \\ -1 & 0 & 0 & 0 & -1 & 0 & 0\end{array}$

$\begin{array}{lllllll}-1 & 0 & -1 & 0 & -1 & -1 & 0\end{array}$

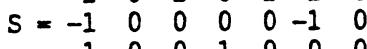

$\begin{array}{rrrrrrr}1 & 0 & 0 & -1 & 0 & 0 & 0 \\ 1 & 1 & 0 & 0 & 1 & 1 & 0 \\ 1 & 0 & 1 & 1 & 1 & 1 & 0\end{array}$

$\begin{array}{lllllll}1 & 0 & 1 & 1 & 1 & 1 & 0\end{array}$

voisine $=\begin{array}{rrrrrrr}4 & 0 & 3 / 2 & 1 / 2 & 1 & 2 & 1 \\ 0 & 4 & 1 & 2 & 2 & 2 & 2 \\ 3 / 2 & 1 & 4 & 3 / 2 & 2 & 2 & 2 \\ 1 / 2 & 2 & 3 / 2 & 4 & 2 & 2 & 2 \\ 1 & 2 & 2 & 2 & 4 & 1 & 3 / 2 \\ 2 & 2 & 2 & 2 & 1 & 4 & 1 \\ 1 & 2 & 2 & 2 & 3 / 2 & 1 & 4\end{array}$

$S=\begin{array}{rrrrrrr}0 & 0 & 0 & 0 & -1 & -1 & 1 \\ 0 & 1 & 0 & 0 & 0 & -1 & 1 \\ 0 & 0 & 0 & 0 & 0 & -1 & 1 \\ 0 & 0 & 0 & 1 & 0 & -1 & 1 \\ 0 & 0 & 1 & 0 & 0 & 1 & -1 \\ 0 & 0 & 0 & 0 & 1 & 2 & -1 \\ 1 & -1 & 0 & 0 & 0 & 1 & -1\end{array}$

$41 \rightarrow$

$16 \times \quad P(7,19)$

Voisine $=\begin{array}{rrrrrrr}4 & 2 / 3 & 2 & 2 / 3 & 2 & 2 & 2 \\ 2 / 3 & 4 & 2 / 3 & 2 & 2 & 2 & 2 \\ 2 & 2 / 3 & 4 & 2 / 3 & 2 & 2 & 2 \\ 2 / 3 & 2 & 2 / 3 & 4 & 2 & 2 & 2 \\ 2 & 2 & 2 & 2 & 4 & 4 / 3 & 2 \\ 2 & 2 & 2 & 2 & 4 / 3 & 4 & 2 \\ 2 & 2 & 2 & 2 & 2 & 2 & 4\end{array}$

$S=\begin{array}{rrrrrrr}0 & 0 & -1 & 0 & 0 & 0 & 0 \\ 0 & 0 & 0 & 0 & -1 & 0 & 0 \\ 0 & 0 & 0 & -1 & 0 & 0 & 0 \\ 0 & 0 & 0 & 0 & 0 & -1 & 0 \\ -1 & -1 & 0 & 0 & 0 & 0 & -1 \\ 0 & 0 & 1 & 1 & 1 & 1 & 1 \\ 1 & 0 & 0 & 0 & 0 & 0 & 0\end{array}$ 
$42 \rightarrow$

$$
\text { voisine }=\begin{array}{rrrrrrr}
4 & 4 / 3 & 2 & 0 & 2 & 2 & 4 / 3 \\
4 / 3 & 4 & 4 / 3 & 4 / 3 & 2 & 2 & 2 \\
2 & 4 / 3 & 4 & 0 & 2 & 2 & 2 \\
0 & 4 / 3 & 0 & 4 & 4 / 3 & 4 / 3 & 4 / 3 \\
2 & 2 & 2 & 4 / 3 & 4 & 2 / 3 & 2 \\
2 & 2 & 2 & 4 / 3 & 2 / 3 & 4 & 2 / 3 \\
4 / 3 & 2 & 2 & 4 / 3 & 2 & 2 / 3 & 4
\end{array}
$$

$43 \rightarrow$

$$
\begin{array}{rrrrrrr}
4 & 2 / 3 & 2 & 2 / 3 & 2 & 2 & 2 \\
2 / 3 & 4 & 2 / 3 & 2 & 2 & 2 & 2 \\
2 & 2 / 3 & 4 & 2 / 3 & 2 & 2 & 4 / 3 \\
2 / 3 & 2 & 2 / 3 & 4 & 2 & 2 & 4 / 3 \\
2 & 2 & 2 & 2 & 4 & 4 / 3 & 4 / 3 \\
2 & 2 & 2 & 2 & 4 / 3 & 4 & 4 / 3 \\
2 & 2 & 4 / 3 & 4 / 3 & 4 / 3 & 4 / 3 & 4
\end{array}
$$

$44 \rightarrow$

$32 \times \quad P(7,23)$

$32 \times \quad P(7,21)$

$$
S=\begin{array}{rrrrrrr}
0 & -1 & -1 & 0 & 0 & 0 & 0 \\
-1 & -1 & 0 & 0 & 0 & 0 & 1 \\
-1 & -1 & -1 & 0 & 1 & 1 & 1 \\
-1 & -1 & -1 & 0 & 0 & 0 & 0 \\
1 & 1 & 1 & 0 & 0 & -1 & -1 \\
1 & 1 & 1 & 0 & 0 & 0 & -1 \\
1 & 1 & 0 & -1 & -1 & 0 & 0
\end{array}
$$

$\begin{array}{rrrrrrr}4 & 2 / 3 & 2 & 2 / 3 & 2 & 2 & 2 \\ 2 / 3 & 4 & 2 / 3 & 2 & 2 & 2 & 2 \\ 2 & 2 / 3 & 4 & 2 / 3 & 2 & 2 & 4 / 3 \\ 2 / 3 & 2 & 2 / 3 & 4 & 2 & 2 & 4 / 3 \\ 2 & 2 & 2 & 2 & 4 & 4 / 3 & 4 / 3 \\ 2 & 2 & 2 & 2 & 4 / 3 & 4 & 4 / 3 \\ 2 & 2 & 4 / 3 & 4 / 3 & 4 / 3 & 4 / 3 & 4\end{array}$

$$
\mathrm{S}=\begin{array}{rrrrrrr}
-1 & 0 & 1 & 0 & 0 & 0 & 0 \\
-1 & 0 & 0 & 0 & -1 & 0 & 0 \\
0 & 0 & 0 & 1 & 0 & 0 & 0 \\
0 & 0 & 0 & 0 & 0 & -1 & 0 \\
0 & 0 & -1 & -1 & 0 & 0 & -1 \\
1 & 1 & 0 & 0 & 1 & 1 & 1 \\
1 & 0 & 0 & 0 & 0 & 0 & 0
\end{array}
$$

$$
44 \rightarrow
$$

$$
\text { voisine }=\begin{array}{rrrrrrr}
4 & 2 / 3 & 2 & 2 / 3 & 2 & 2 & 2 \\
2 / 3 & 4 & 2 / 3 & 2 & 4 / 3 & 2 & 2 \\
2 & 2 / 3 & 4 & 2 / 3 & 2 & 2 & 4 / 3 \\
2 / 3 & 2 & 2 / 3 & 4 & 2 & 4 / 3 & 2 \\
2 & 4 / 3 & 2 & 2 & 4 & 2 / 3 & 4 / 3 \\
2 & 2 & 2 & 4 / 3 & 2 / 3 & 4 & 4 / 3 \\
2 & 2 & 4 / 3 & 2 & 4 / 3 & 4 / 3 & 4
\end{array}
$$$$
S=\begin{array}{rrrrrrr}
0 & 0 & 0 & -1 & -1 & 1 & 0 \\
0 & 1 & 0 & 0 & 0 & 1 & 1 \\
0 & 0 & -1 & 0 & 0 & 0 & 0 \\
1 & 0 & 0 & 0 & 0 & 1 & 0 \\
0 & 0 & 0 & 0 & 0 & -1 & 0 \\
0 & 0 & 0 & 0 & 0 & -1 & -1 \\
0 & 0 & 0 & 0 & 1 & -1 & 0
\end{array}
$$

$45 \rightarrow$

$$
128 \times \quad P(7,24)
$$

$$
\text { voisine }=\begin{array}{rrrrrrr}
4 & 2 / 3 & 2 & 0 & 2 & 4 / 3 & 4 / 3 \\
2 / 3 & 4 & 4 / 3 & 4 / 3 & 2 & 2 & 2 \\
2 & 4 / 3 & 4 & 2 / 3 & 2 & 2 & 2 \\
0 & 4 / 3 & 2 / 3 & 4 & 4 / 3 & 2 & 4 / 3 \\
2 & 2 & 2 & 4 / 3 & 4 & 2 / 3 & 2 \\
4 / 3 & 2 & 2 & 2 & 2 / 3 & 4 & 2 / 3 \\
4 / 3 & 2 & 2 & 4 / 3 & 2 & 2 / 3 & 4
\end{array}
$$

$46 \rightarrow$

$128 \times \quad P(7,24)$

$$
\text { voisine }=\begin{array}{rrrrrrr}
4 & 2 / 3 & 4 / 3 & 2 / 3 & 2 & 2 & 2 \\
2 / 3 & 4 & 4 / 3 & 2 & 2 & 2 & 2 \\
4 / 3 & 4 / 3 & 4 & 2 \beta & 2 & 2 & 2 \\
2 / 3 & 2 & 2 \beta & 4 & 2 & 2 & 4 / 3 \\
2 & 2 & 2 & 2 & 4 & 4 / 3 & 2 \\
2 & 2 & 2 & 2 & 4 / 3 & 4 & 4 / 3 \\
2 & 2 & 2 & 4 / 3 & 2 & 4 / 3 & 4
\end{array}
$$

Nambre total de voisines : 2688

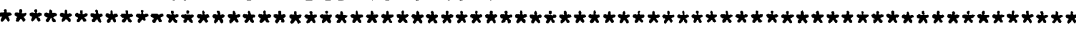

Forme parfaite $P(7,6)$.

$\begin{array}{lllllll}6 & 2 & 2 & 2 & 3 & 3 & 3 \\ 2 & 6 & 2 & 2 & 3 & 3 & 3 \\ 2 & 2 & 6 & 2 & 3 & 3 & 3 \\ 2 & 2 & 2 & 6 & 3 & 3 & 3 \\ 3 & 3 & 3 & 3 & 6 & 2 & 3 \\ 3 & 3 & 3 & 3 & 2 & 6 & 1 \\ 3 & 3 & 3 & 3 & 3 & 1 & 6\end{array}$

Nombre de vecteurs minimaux : 32

Minimm

: 6

Déterminant

: 9216

Déterminant (minimum normalisé à 2) : 1024/243

Ordre du groupe des automorphismes : 192 
Liste des orbites des voisines de la forme $P(7,6)$ :

$\begin{array}{rrr}1 \rightarrow & 4 \times & P(7,1) \\ 2 \rightarrow & 8 \times & P(7,1) \\ 3 \rightarrow & 8 \times & P(7,1) \\ 4 \rightarrow & 8 \times & P(7,1) \\ 5 \rightarrow & 12 \times & P(7,1) \\ 6 \rightarrow & 16 \times & P(7,1) \\ 7 \rightarrow & 4 \times & P(7,3) \\ 8 \rightarrow & 12 \times & P(7,3) \\ 9 \rightarrow & 16 \times & P(7,3) \\ 10 \rightarrow & 3 \times & P(7,5) \\ 11 \rightarrow & 1 \times \times & P(7,6) \\ 12 \rightarrow & 16 \times & P(7,6) \\ 13 \rightarrow & 12 \times & P(7,7) \\ 14 \rightarrow & 24 \times & P(7,7) \\ 15 \rightarrow & 24 \times & P(7,8) \\ 16 \rightarrow & 4 \times & P(7,9) \\ 17 \rightarrow & 24 \times & P(7,14) \\ 18 \rightarrow & 24 \times & P(7,16) \\ 19 \rightarrow & 48 \times & P(7,17)\end{array}$

Nombre total de voisines : 268

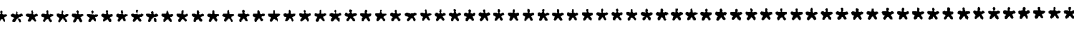

Forme parfaite $P(7,7)$.

$\begin{array}{lllllll}4 & 2 & 2 & 0 & 1 & 1 & 1 \\ 2 & 4 & 2 & 1 & 0 & 1 & 2 \\ 2 & 2 & 4 & 1 & 1 & 0 & 2 \\ 0 & 1 & 1 & 4 & 2 & 2 & 1 \\ 1 & 0 & 1 & 2 & 4 & 2 & 2 \\ 1 & 1 & 0 & 2 & 2 & 4 & 2 \\ 1 & 2 & 2 & 1 & 2 & 2 & 4\end{array}$

Nombre de vecteurs minimaux : 34 Minimum

: 4

Déterminant

: 540

Déterminant (minimum normalisé à 2) : 135/32

Ordre du groupe des automorphismes : 48

Liste des orbites des voisines de la forme $P(7,7)$ :

$1 \rightarrow$

$2 \times \quad P(7,1)$

Voisine = $\begin{array}{rrrrrrr}4 & 2 & 2 & 2 & 2 & 2 & 0 \\ 2 & 4 & 2 & 2 & 2 & 2 & 2 \\ 2 & 2 & 4 & 2 & 2 & 2 & 2 \\ 2 & 2 & 2 & 4 & 2 & 2 & 0 \\ 2 & -2 & 2 & 2 & 4 & 2 & 2 \\ 2 & 2 & 2 & 2 & 2 & 4 & 2 \\ 0 & 2 & 2 & 0 & 2 & 2 & 4\end{array}$

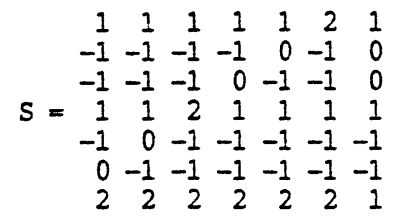

$2 \rightarrow$

$6 \times \quad P(7,1)$

Voisine = $\begin{array}{lllllll}4 & 2 & 0 & 0 & 0 & 2 & 0 \\ 2 & 4 & 2 & 2 & 0 & 2 & 2 \\ 0 & 2 & 4 & 2 & 0 & 0 & 2 \\ 0 & 2 & 2 & 4 & 2 & 2 & 2 \\ 0 & 0 & 0 & 2 & 4 & 2 & 2 \\ 2 & 2 & 0 & 2 & 2 & 4 & 2 \\ 0 & 2 & 2 & 2 & 2 & 2 & 4\end{array}$

$S=\begin{array}{rrrrrrr}1 & 0 & 0 & 1 & 0 & 0 & 0 \\ -1 & -1 & 0 & -1 & -1 & 0 & 0 \\ -1 & 0 & 0 & 0 & 0 & -1 & 0 \\ 1 & 0 & 0 & 0 & 0 & 0 & 0 \\ -1 & -1 & 0 & 0 & 0 & 0 & 1 \\ -1 & 1 & 0 & 0 & 0 & 0 & 0 \\ 2 & 1 & 1 & 1 & 1 & 1 & 0\end{array}$


$3 \rightarrow$

Voisine $=\begin{array}{rrrrrrr}4 & 2 & 2 & 0 & 0 & 0 & 0 \\ 2 & 4 & 2 & 0 & 0 & 0 & 2 \\ 2 & 2 & 4 & 0 & 0 & -2 & 0 \\ 0 & 0 & 0 & 4 & 2 & 2 & 0 \\ 0 & 0 & 0 & 2 & 4 & 2 & 2 \\ 0 & 0 & -2 & 2 & 2 & 4 & 2 \\ 0 & 2 & 0 & 0 & 2 & 2 & 4\end{array}$

$4 \rightarrow \quad 12 \times \quad P(7,1)$

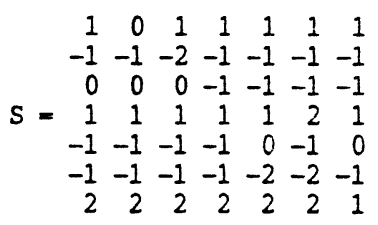

$\begin{array}{llllllllll}1 & 1 & 0 & 1 & 1 & 1 & 0\end{array}$ $\begin{array}{lllllll}-1 & -1 & -1 & -2 & -1 & -1 & -1\end{array}$ $\begin{array}{lllllll}-1 & 0 & 0 & 0 & -1 & -1 & 0\end{array}$ $S=\begin{array}{rrrrrrr}2 & 1 & 1 & 1 & 1 & 2 & 0\end{array}$ $\begin{array}{lllllll}-1 & -1 & -1 & -1 & -1 & -2 & -1\end{array}$ $\begin{array}{lllllll}-1 & -1 & -1 & -1 & -1 & -1 & 0\end{array}$

$5 \rightarrow \quad 12 \times \quad P(7,1)$

$\begin{array}{lllllll}4 & 2 & 2 & 0 & 2 & 2 & 2 \\ 2 & 4 & 2 & 2 & 2 & 2 & 2 \\ 2 & 2 & 4 & 0 & 2 & 0 & 2 \\ \text { Voisine }= & 2 & 0 & 4 & 2 & 2 & 0 \\ 2 & 2 & 2 & 2 & 4 & 2 & 2 \\ 2 & 2 & 0 & 2 & 2 & 4 & 2 \\ 2 & 2 & 2 & 0 & 2 & 2 & 4\end{array}$

$6 \rightarrow \quad 12 \times \quad P(7,1)$

Voisine $=\quad \begin{array}{lllllll}8 & 4 & 4 & 2 & 4 & 2 & 2 \\ 4 & 4 & 2 & 2 & 2 & 2 & 2 \\ 4 & 2 & 4 & 0 & 2 & 0 & 2 \\ 2 & 2 & 0 & 4 & 2 & 2 & 0 \\ 4 & 2 & 2 & 2 & 4 & 2 & 2 \\ 2 & 2 & 0 & 2 & 2 & 4 & 2 \\ 2 & 2 & 2 & 0 & 2 & 2 & 4\end{array}$

$S=\begin{array}{rrrrrrr}1 & 0 & 0 & 0 & 0 & 1 & 0 \\ -1 & -1 & 0 & -1 & 0 & -1 & 0 \\ -1 & 0 & 0 & 0 & -1 & -1 & 0 \\ 1 & 1 & 0 & 0 & 0 & 0 & -1 \\ -1 & 0 & 0 & 0 & 0 & 0 & 0 \\ -1 & 0 & 0 & 0 & 0 & 0 & 1 \\ 2 & 1 & 1 & 1 & 1 & 1 & 0\end{array}$

$7 \rightarrow \quad 12 \times \quad P(7,1)$

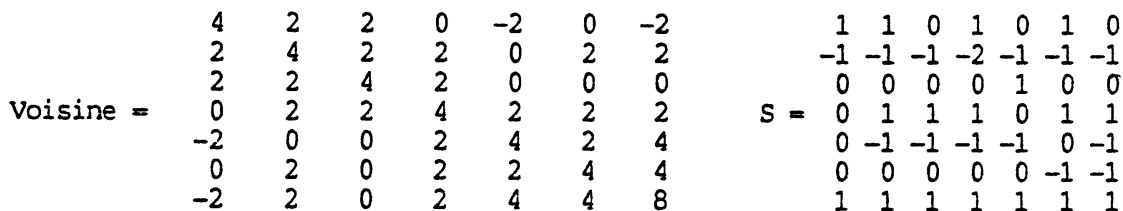

$8 \rightarrow \quad 12 \times \quad P(7,1)$

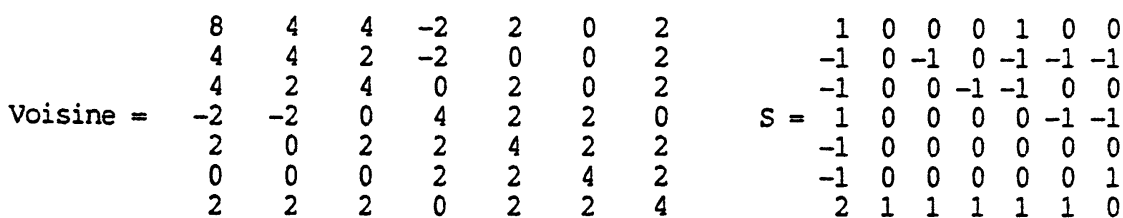


$9 \rightarrow$

$\begin{array}{llllllll}4 & 4 & 4 & 2 & 2 & 2 & 2 \\ \text { Voisine }= & 8 & 6 & 4 & 2 & 2 & 4 \\ 4 & 6 & 8 & 4 & 2 & 2 & 4 \\ 2 & 4 & 4 & 4 & 2 & 2 & 2 \\ 2 & 2 & 2 & 2 & 4 & 2 & 2 \\ 2 & 2 & 2 & 2 & 2 & 4 & 2 \\ 2 & 4 & 4 & 2 & 2 & 2 & 4\end{array}$

$10 \rightarrow \quad 12 \times \quad P(7,1)$

Voisine = $\begin{array}{lllllll}4 & 2 & 2 & 0 & 0 & 0 & 0 \\ 2 & 4 & 2 & 2 & 2 & 2 & 2 \\ 2 & 2 & 4 & 0 & 0 & 0 & 2 \\ 0 & 2 & 0 & 4 & 2 & 2 & 0 \\ 0 & 2 & 0 & 2 & 4 & 2 & 2 \\ 0 & 2 & 0 & 2 & 2 & 4 & 2 \\ 0 & 2 & 2 & 0 & 2 & 2 & 4\end{array}$

$11 \rightarrow \quad 12 \times \quad P(7,1)$

$\begin{array}{rrrrrrrr}4 & 2 & 2 & -2 & -2 & -2 & 0 \\ 2 & 4 & 2 & 0 & -2 & 0 & 2 \\ 2 & 2 & 4 & 0 & -2 & -2 & 0 \\ -2 & 0 & 0 & 4 & 2 & 2 & 0 \\ -2 & -2 & -2 & 2 & 4 & 2 & 0 \\ -2 & 0 & -2 & 2 & 2 & 4 & 2 \\ 0 & 2 & 0 & 0 & 0 & 2 & 4\end{array}$

$12 \rightarrow \quad 24 \times \quad P(7,1)$

$$
\begin{array}{llllllll}
4 & 2 & 4 & 2 & 2 & 2 & 2 \\
2 & 4 & 4 & 2 & 0 & 2 & 4 \\
4 & 4 & 8 & 4 & 2 & 2 & 4 \\
2 & 2 & 4 & 4 & 2 & 2 & 2 \\
2 & 0 & 2 & 2 & 4 & 2 & 2 \\
2 & 2 & 2 & 2 & 2 & 4 & 4 \\
2 & 4 & 4 & 2 & 2 & 4 & 8
\end{array}
$$

$13 \rightarrow$

$$
\text { Voisine = } \begin{array}{lllllll}
4 & 4 & 4 & 0 & 0 & 2 & 2 \\
4 & 8 & 6 & 2 & 0 & 2 & 4 \\
4 & 6 & 8 & 2 & 0 & 2 & 4 \\
0 & 2 & 2 & 4 & 2 & 2 & 2 \\
0 & 0 & 0 & 2 & 4 & 2 & 2 \\
2 & 2 & 2 & 2 & 2 & 4 & 2 \\
2 & 4 & 4 & 2 & 2 & 2 & 4
\end{array}
$$

$14 \rightarrow$

$$
\text { Voisine = } \begin{array}{lllllll}
4 & 4 & 4 & 0 & 0 & 0 & 2 \\
4 & 8 & 6 & 2 & 0 & 2 & 6 \\
4 & 6 & 8 & 2 & 0 & 0 & 4 \\
0 & 2 & 2 & 4 & 2 & 2 & 2 \\
0 & 0 & 0 & 2 & 4 & 2 & 2 \\
0 & 2 & 0 & 2 & 2 & 4 & 4 \\
2 & 6 & 4 & 2 & 2 & 4 & 8
\end{array}
$$
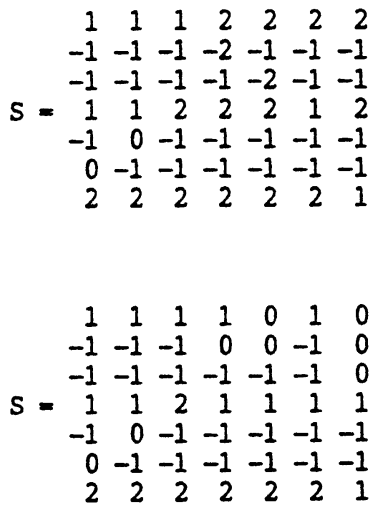

$$
S=\begin{array}{rrrrrrr}
0 & 0 & 1 & 0 & 0 & 0 & 1 \\
-1 & -1 & -2 & -2 & -2 & -1 & -2 \\
0 & 0 & 0 & 0 & 1 & 0 & 0 \\
0 & 1 & 1 & 1 & 0 & 1 & 1 \\
0 & -1 & -1 & -1 & -1 & 0 & -1 \\
0 & 0 & 0 & 0 & 0 & -1 & -1 \\
1 & 1 & 1 & 1 & 1 & 1 & 1
\end{array}
$$


$15 \rightarrow$

$$
\begin{array}{llllllll}
4 & 2 & 2 & 0 & 2 & 2 & 2 \\
2 & 4 & 2 & 0 & 0 & 0 & 2 \\
2 & 2 & 4 & 0 & 0 & 0 & 2 \\
0 & 0 & 0 & 4 & 4 & 2 & 0 \\
2 & 0 & 0 & 4 & 8 & 4 & 2 \\
2 & 0 & 0 & 2 & 4 & 4 & 2 \\
2 & 2 & 2 & 0 & 2 & 2 & 4
\end{array}
$$

$16 \rightarrow$

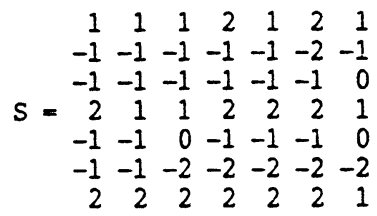

$\mathbf{S}=\begin{array}{rrrrrrr}1 & 0 & 0 & 0 & 1 & 0 & 0 \\ -1 & 0 & -1 & 0 & -1 & -1 & -1 \\ -1 & 0 & 1 & 0 & 0 & 0 & 1 \\ 2 & 0 & 1 & 1 & 1 & 1 & 1 \\ -1 & 0 & -1 & -1 & -1 & -1 & -1 \\ -2 & 0 & 0 & 0 & 0 & 0 & 1 \\ 2 & 1 & 1 & 1 & 1 & 1 & 0\end{array}$

$17->$

$24 \times \quad P(7,1)$

$\begin{array}{llllllll}4 & 4 & 4 & 0 & 0 & 0 & 2 \\ \text { Voisine }= & 8 & 6 & 4 & 2 & 2 & 4 \\ 4 & 6 & 8 & 2 & 2 & 0 & 4 \\ 0 & 4 & 2 & 8 & 4 & 4 & 2 \\ 0 & 2 & 2 & 4 & 4 & 2 & 2 \\ 0 & 2 & 0 & 4 & 2 & 4 & 2 \\ 2 & 4 & 4 & 2 & 2 & 2 & 4\end{array}$

$18 \rightarrow$

$24 \times \quad P(7,1)$

$\begin{array}{llllllll}4 & 2 & 2 & 0 & 2 & 2 & 2 \\ \text { voisine }= & 4 & 2 & 2 & 2 & 2 & 2 \\ 2 & 2 & 4 & 0 & 2 & 0 & 2 \\ 0 & 2 & 0 & 8 & 6 & 6 & 2 \\ 2 & 2 & 2 & 6 & 8 & 6 & 4 \\ 2 & 2 & 0 & 6 & 6 & 8 & 4 \\ 2 & 2 & 2 & 2 & 4 & 4 & 4\end{array}$

$19 \rightarrow$

$2 \times \quad P(7,3)$

Voisine = $\begin{array}{lllllll}4 & 2 & 2 & 1 & 2 & 2 & 1 \\ 2 & 4 & 2 & 2 & 1 & 2 & 2 \\ 2 & 2 & 4 & 2 & 2 & 1 & 2 \\ 1 & 2 & 2 & 4 & 2 & 2 & 1 \\ 2 & 1 & 2 & 2 & 4 & 2 & 2 \\ 2 & 2 & 1 & 2 & 2 & 4 & 2 \\ 1 & 2 & 2 & 1 & 2 & 2 & 4\end{array}$

$20 \rightarrow$

$6 \times \quad P(7,3)$

Voisine = $\begin{array}{rrrrrrr}4 & 2 & 2 & 1 & 2 & 1 & 1 \\ 2 & 4 & 2 & 2 & 1 & 1 & 2 \\ 2 & 2 & 4 & 1 & 1 & -1 & 1 \\ 1 & 2 & 1 & 4 & 2 & 2 & 1 \\ 2 & 1 & 1 & 2 & 4 & 2 & 2 \\ 1 & 1 & -1 & 2 & 2 & 4 & 2 \\ 1 & 2 & 1 & 1 & 2 & 2 & 4\end{array}$
$S=\begin{array}{rrrrrrr}1 & 0 & 0 & 0 & 0 & 0 & 0 \\ -1 & 0 & -1 & 0 & 0 & 0 & 0 \\ -1 & -1 & 0 & -1 & -1 & 0 & 0 \\ 1 & 0 & 1 & 1 & 1 & 0 & 1 \\ 0 & 0 & -1 & -1 & 0 & 0 & -1 \\ -1 & -1 & -1 & -1 & -2 & 0 & -1 \\ 2 & 2 & 2 & 2 & 2 & 1 & 1\end{array}$

$S=\begin{array}{rrrrrrr}1 & 0 & 0 & 1 & 1 & 1 & 1 \\ -1 & 0 & 0 & -1 & 0 & 0 & 0 \\ -1 & -1 & 0 & 0 & -1 & -1 & 0 \\ 2 & 0 & 1 & 1 & 1 & 1 & 1 \\ -1 & 0 & -1 & -1 & -1 & 0 & -1 \\ -2 & -1 & -1 & -1 & -1 & -2 & -1 \\ 3 & 2 & 2 & 2 & 2 & 2 & 1\end{array}$

$S=\begin{array}{rrrrrrr}0 & 0 & 1 & 0 & 0 & 1 & 0 \\ 0 & 0 & -1 & 0 & 1 & 0 & 0 \\ 0 & 0 & 0 & 0 & 0 & -1 & 0 \\ 0 & 1 & 1 & 0 & 0 & 1 & 0 \\ 0 & 0 & -1 & 1 & 0 & 0 & 0 \\ 0 & 0 & 0 & 0 & 0 & -1 & 1 \\ 1 & 0 & 1 & 0 & 0 & 1 & 0\end{array}$

$\mathbf{S}=\begin{array}{rrrrrrr}0 & 0 & 0 & 0 & 0 & 1 & 0 \\ -1 & 0 & 0 & 0 & -1 & -1 & 0 \\ 0 & 0 & 0 & 0 & 1 & 0 & 0 \\ 1 & 0 & 0 & 1 & 0 & 1 & 0 \\ 0 & 0 & 0 & 0 & 0 & -1 & 1 \\ -1 & -1 & -1 & -1 & 0 & -1 & -1 \\ 1 & 0 & 1 & 0 & 0 & 1 & 0\end{array}$ 
$21 \rightarrow$ $\begin{array}{llllllll}4 & 3 & 2 & 1 & 2 & 2 & 2 \\ \text { Voisine }= & 6 & 3 & 3 & 2 & 3 & 4 \\ 2 & 3 & 4 & 2 & 2 & 1 & 3 \\ 1 & 3 & 2 & 4 & 2 & 2 & 2 \\ 2 & 2 & 2 & 2 & 4 & 2 & 3 \\ 2 & 3 & 1 & 2 & 2 & 4 & 3 \\ 2 & 4 & 3 & 2 & 3 & 3 & 6\end{array}$

$22 \rightarrow$ Voisine = $\begin{array}{rrrrrrr}4 & 2 & 2 & 0 & 1 & 1 & 1 \\ 2 & 4 & 2 & 0 & 0 & 0 & 2 \\ 2 & 2 & 4 & 0 & 1 & -1 & 2 \\ 0 & 0 & 0 & 4 & 2 & 2 & 0 \\ 1 & 0 & 1 & 2 & 4 & 2 & 2 \\ 1 & 0 & -1 & 2 & 2 & 4 & 1 \\ 1 & 2 & 2 & 0 & 2 & 1 & 4\end{array}$ $23 \rightarrow$ $\begin{array}{rrrrrrr}4 & 2 & 2 & -1 & 0 & 1 & 1 \\ 2 & 4 & 2 & 1 & 0 & 2 & 3 \\ 2 & 2 & 4 & 0 & 0 & 0 & 2 \\ -1 & 1 & 0 & 4 & 2 & 2 & 1 \\ 0 & 0 & 0 & 2 & 4 & 2 & 2 \\ 1 & 2 & 0 & 2 & 2 & 4 & 3 \\ 1 & 3 & 2 & 1 & 2 & 3 & 6\end{array}$ $24 \rightarrow$ voisine = $\begin{array}{lllllll}4 & 2 & 2 & 0 & 1 & 1 & 1 \\ 2 & 4 & 2 & 1 & 0 & 2 & 3 \\ 2 & 2 & 4 & 1 & 0 & 0 & 1 \\ 0 & 1 & 1 & 4 & 2 & 3 & 1 \\ 1 & 0 & 0 & 2 & 4 & 3 & 2 \\ 1 & 2 & 0 & 3 & 3 & 6 & 4 \\ 1 & 3 & 1 & 1 & 2 & 4 & 6\end{array}$ $25 \rightarrow$ Voisine = $\begin{array}{lllllll}4 & 3 & 3 & 0 & 0 & 1 & 1 \\ 3 & 6 & 4 & 2 & 0 & 1 & 3 \\ 3 & 4 & 6 & 2 & 1 & 1 & 3 \\ 0 & 2 & 2 & 4 & 2 & 2 & 2 \\ 0 & 0 & 1 & 2 & 4 & 2 & 2 \\ 1 & 1 & 1 & 2 & 2 & 4 & 2 \\ 1 & 3 & 3 & 2 & 2 & 2 & 4\end{array}$ $26 \rightarrow \quad 24 \times \quad P(7,5)$ voisine $=\begin{array}{lllllll}4 & 3 & 2 & 1 & 0 & 1 & 0 \\ 3 & 6 & 3 & 2 & 0 & 1 & 2 \\ 2 & 3 & 4 & 2 & 0 & 0 & 1 \\ 1 & 2 & 2 & 4 & 2 & 2 & 1 \\ 0 & 0 & 0 & 2 & 4 & 2 & 2 \\ 1 & 1 & 0 & 2 & 2 & 4 & 2 \\ 0 & 2 & 1 & 1 & 2 & 2 & 4\end{array}$

$$
12 \times \quad P(7,5)
$$

$24 \times \quad P(7,5)$

$$
24 \times \quad P(7,5)
$$

$$
S=\begin{array}{rrrrrrr}
1 & 0 & 0 & 0 & 1 & 0 & 1 \\
-1 & 0 & -1 & 0 & 0 & 0 & -1 \\
0 & 1 & 1 & 0 & 0 & 0 & 0 \\
1 & 0 & 0 & 1 & 0 & 0 & 1 \\
-1 & 0 & 0 & 0 & 0 & 0 & 0 \\
0 & 1 & 1 & 0 & 0 & 1 & 0 \\
1 & -1 & 0 & 0 & 0 & 0 & 0
\end{array}
$$

$$
\mathbf{S}=\begin{array}{rrrrrrr}
0 & 0 & -1 & 1 & 0 & 0 & 0 \\
0 & 0 & 1 & 0 & 0 & 0 & 0 \\
-1 & 0 & 0 & -1 & 0 & -1 & 0 \\
0 & 0 & -1 & 0 & 0 & 0 & -1 \\
0 & -1 & 1 & 0 & 0 & 0 & 0 \\
0 & 1 & 1 & 0 & 1 & 0 & 1 \\
1 & 0 & -1 & 0 & 0 & 0 & 0
\end{array}
$$


$27 \rightarrow$ $\begin{array}{llllllll}4 & 2 & 2 & 0 & 1 & 2 & 2 \\ 2 & 4 & 2 & 1 & 0 & 1 & 2 \\ 2 & 2 & 4 & 0 & 0 & 0 & 2 \\ 0 & 1 & 0 & 4 & 2 & 2 & 0 \\ 1 & 0 & 0 & 2 & 4 & 2 & 1 \\ 2 & 1 & 0 & 2 & 2 & 4 & 2 \\ 2 & 2 & 2 & 0 & 1 & 2 & 4\end{array}$ $28 \rightarrow$ $24 \times \quad P(7,5)$

$$
S=\begin{array}{rrrrrrr}
0 & 1 & 1 & 0 & 1 & 0 & 1 \\
-1 & 0 & -1 & 0 & -1 & 0 & -1 \\
0 & -1 & 0 & 0 & 0 & 0 & -1 \\
1 & 1 & 1 & 0 & 1 & 0 & 1 \\
-1 & -1 & -1 & -1 & -1 & -1 & -1 \\
0 & -1 & 0 & 0 & -1 & 1 & -1 \\
1 & 1 & 0 & 0 & 1 & 0 & 1
\end{array}
$$

$$
\text { Voisine = } \begin{array}{lllllll}
4 & 3 & 2 & 0 & 0 & 1 & 1 \\
3 & 6 & 3 & 2 & 0 & 1 & 2 \\
2 & 3 & 4 & 1 & 0 & 0 & 2 \\
0 & 2 & 1 & 4 & 2 & 2 & 1 \\
0 & 0 & 0 & 2 & 4 & 2 & 2 \\
1 & 1 & 0 & 2 & 2 & 4 & 2 \\
1 & 2 & 2 & 1 & 2 & 2 & 4
\end{array}
$$

$29 \rightarrow$

$$
3 \times \quad P(7,6)
$$

$$
\begin{array}{rrrrrrr}
4 & 2 & 2 & 4 / 3 & 2 & 2 & 4 / 3 \\
2 & 4 & 2 & 2 & 2 / 3 & 4 / 3 & 2 \\
2 & 2 & 4 & 2 & 4 / 3 & 2 / 3 & 2 \\
4 / 3 & 2 & 2 & 4 & 2 & 2 & 4 / 3 \\
2 & 2 / 3 & 4 / 3 & 2 & 4 & 2 & 2 \\
2 & 4 / 3 & 2 / 3 & 2 & 2 & 4 & 2 \\
4 / 3 & 2 & 2 & 4 / 3 & 2 & 2 & 4
\end{array}
$$$$
S=\begin{array}{rrrrrrr}
0 & -1 & 0 & 1 & 0 & 0 & 0 \\
0 & 1 & 0 & 0 & 0 & 1 & 0 \\
0 & 1 & 0 & 0 & 0 & 0 & 0 \\
0 & -1 & 1 & 0 & 0 & 0 & 0 \\
0 & 1 & 0 & 0 & 0 & 0 & 1 \\
0 & 1 & 0 & 0 & 1 & 0 & 0 \\
1 & -1 & 0 & 0 & 0 & 0 & 0
\end{array}
$$

$30 \rightarrow$

$6 \times \quad P(7,6)$

$$
\begin{array}{rrrrrrrr}
4 & 2 & 2 & 2 / 3 & 2 & 4 / 3 & 4 / 3 \\
\text { Voisine }= & 2 & 4 & 2 & 4 / 3 & 2 / 3 & 2 / 3 & 2 \\
2 & 2 & 4 & 2 / 3 & 4 / 3 & -2 / 3 & 2 \\
2 / 3 & 4 / 3 & 2 / 3 & 4 & 2 & 2 & 2 / 3 \\
2 & 2 / 3 & 4 / 3 & 2 & 4 & 2 & 2 \\
4 / 3 & 2 / 3 & -2 / 3 & 2 & 2 & 4 & 4 / 3 \\
4 / 3 & 2 & 2 & 2 / 3 & 2 & 4 / 3 & 4
\end{array}
$$$$
S=\begin{array}{rrrrrrr}
0 & -1 & 0 & 1 & 0 & 0 & 0 \\
0 & 1 & 0 & 0 & 0 & 1 & 0 \\
-1 & 0 & 0 & -1 & 0 & -1 & 0 \\
0 & -1 & 0 & 0 & 0 & 0 & -1 \\
0 & 1 & -1 & 0 & 0 & 0 & 0 \\
0 & 1 & 1 & 0 & 1 & 0 & 1 \\
1 & -1 & 0 & 0 & 0 & 0 & 0
\end{array}
$$

$31 \rightarrow$

$3 \times \quad P(7,7)$

Voisine $=\quad \begin{aligned} & 4 \\ & 2 \\ & 2 \\ & 1 \\ & 1 \\ & 2 \\ & 1\end{aligned}$

$\begin{array}{lllllll}4 & 2 & 2 & 1 & 1 & 2 & 1 \\ 2 & 4 & 2 & 1 & 0 & 1 & 2 \\ 2 & 2 & 4 & 2 & 1 & 1 & 2 \\ 1 & 1 & 2 & 4 & 2 & 2 & 1 \\ 1 & 0 & 1 & 2 & 4 & 2 & 2 \\ 2 & 1 & 1 & 2 & 2 & 4 & 2 \\ 1 & 2 & 2 & 1 & 2 & 2 & 4\end{array}$

$32 \rightarrow$

$$
4 \times \quad P(7,7)
$$

$$
\text { Voisine = } \begin{array}{lllllll}
4 & 2 & 2 & 2 & 3 & 3 & 1 \\
2 & 4 & 2 & 3 & 2 & 3 & 2 \\
2 & 2 & 4 & 3 & 3 & 2 & 2 \\
2 & 3 & 3 & 8 & 6 & 6 & 3 \\
3 & 2 & 3 & 6 & 8 & 6 & 4 \\
3 & 3 & 2 & 6 & 6 & 8 & 4 \\
1 & 2 & 2 & 3 & 4 & 4 & 4
\end{array}
$$

$\begin{array}{rrrrrrr}0 & -1 & -1 & -1 & -1 & -1 & -1\end{array}$

$\begin{array}{lllllll}0 & 1 & 1 & 1 & 1 & 1 & 0\end{array}$

$S=\begin{array}{lllllll}1 & -1 & 0 & -1 & 0 & -1 & 0\end{array}$

$\begin{array}{lllllll}-1 & 0 & 0 & 1 & 0 & 0 & 0\end{array}$

$\begin{array}{rrrrrrr}0 & 1 & 0 & 1 & 1 & 1 & 0 \\ 1 & -1 & 0 & -1 & -1 & 0 & 0\end{array}$ 
$33 \rightarrow$ $6 \times \quad P(7,7)$

$\begin{array}{rrrrrrrrrrrrrrr}4 & 3 & 2 & 0 & 1 & 2 & 2 & & -1 & 0 & -1 & 0 & -1 & 0 & -1 \\ \text { Voisine = } & 3 & 6 & 3 & 2 & 1 & 3 & 4 & & 0 & 0 & 0 & 1 & 0 & 1 \\ 2 & 3 & 4 & 1 & 1 & 1 & 3 & 0 & -1 & 0 & 0 & 0 & 0 & 0 \\ 0 & 2 & 1 & 4 & 2 & 3 & 2 & S=-1 & 0 & 0 & 0 & -1 & -1 & -1 \\ 1 & 1 & 1 & 2 & 4 & 3 & 3 & 0 & 0 & 0 & -1 & 0 & 0 & 1 \\ 2 & 3 & 1 & 3 & 3 & 6 & 4 & & 1 & 0 & 0 & 0 & 0 & 0 & 0 \\ 2 & 4 & 3 & 2 & 3 & 4 & 6 & & 0 & 0 & 0 & 0 & 0 & 0 & -1\end{array}$

$34 \rightarrow \quad 12 \times \quad P(7,7)$

Voisine = $\begin{array}{rrrrrrrrrrrrrrrrr}6 & 3 & 4 & 0 & 2 & 1 & 2 & & 1 & 1 & 1 & 0 & 0 & 1 & 1 \\ 3 & 4 & 3 & 0 & 0 & 0 & 2 & & -1 & -1 & -1 & 1 & 0 & 0 & -1 \\ 4 & 3 & 6 & 1 & 2 & 0 & 3 & & -1 & 0 & 0 & 0 & 0 & -1 & 0 \\ 0 & 0 & 1 & 4 & 2 & 2 & 0 & S= & 0 & 1 & 0 & 1 & 1 & 1 \\ 2 & 0 & 2 & 2 & 4 & 2 & 2 & & -1 & -1 & -1 & 0 & -1 & -1 & -1 \\ 1 & 0 & 0 & 2 & 2 & 4 & 1 & & -1 & 0 & -1 & 0 & 0 & 0 & 0 \\ 2 & 2 & 3 & 0 & 2 & 1 & 4 & & 2 & 1 & 1 & 0 & 1 & 1 & 1\end{array}$

$35 \rightarrow \quad 12 \times \quad P(7,7)$

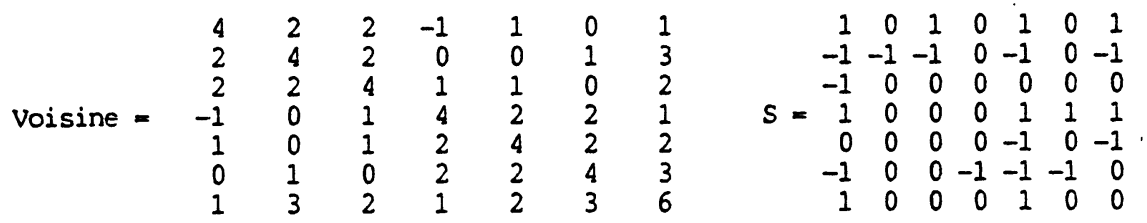

$36 \rightarrow \quad 12 \times \quad P(7,7)$

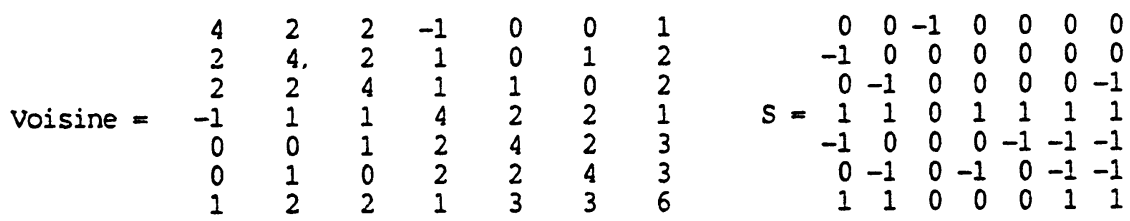

$37 \rightarrow \quad 12 \times \quad P(7,7)$

$\begin{array}{rrrrrrrrrrrrrrrr}4 & 2 & 2 & -1 & 1 & 1 & 1 & & 0 & 0 & 0 & 0 & 0 & 0 & -1 \\ 2 & 4 & 2 & -1 & -1 & 0 & 2 & & 0 & 0 & 1 & 0 & 0 & 0 & 1 \\ \text { Voisine }= & 2 & 2 & 4 & -1 & 0 & -1 & 2 & & 0 & 1 & 0 & 0 & 0 & 0 & 1 \\ -1 & -1 & -1 & 4 & 2 & 2 & 0 & S= & 0 & 0 & 0 & -1 & -1 & -1 & -1 \\ 1 & -1 & 0 & 2 & 4 & 2 & 1 & & 0 & 0 & 0 & 0 & 1 & 1 \\ 1 & 0 & -1 & 2 & 2 & 4 & 1 & & 0 & 0 & 0 & 0 & 1 & 0 & 1 \\ 1 & 2 & 2 & 0 & 1 & 1 & 4 & & 1 & 0 & 0 & 0 & 0 & 0 & -1\end{array}$

$38 \rightarrow \quad 12 \times \quad P(7,7)$

voisine = $\begin{array}{rrrrrrrrrrrrrrrrr}6 & 3 & 3 & -1 & 1 & 1 & 1 & & 0 & 0 & 1 & 1 & 1 & 0 & 1 \\ 3 & 4 & 2 & 0 & 0 & 1 & 2 & & -1 & 0 & -1 & 0 & -1 & 0 & -1 \\ 3 & 2 & 4 & 0 & 1 & 0 & 2 & & 0 & -1 & -1 & -1 & 0 & 0 & -1 \\ -1 & 0 & 0 & 4 & 2 & 2 & 1 & S= & 0 & 0 & 0 & 1 & 1 & 1 & 1 \\ 1 & 0 & 1 & 2 & 4 & 2 & 2 & & 0 & 0 & 0 & 0 & -1 & 0 & -1 \\ 1 & 1 & 0 & 2 & 2 & 4 & 2 & & 0 & 0 & 0 & -1 & 0 & 0 & 0 \\ 1 & 2 & 2 & 1 & 2 & 2 & 4 & & 1 & 1 & 1 & 1 & 1 & 0 & 1\end{array}$ 
$39 \rightarrow$

voisine = $\begin{array}{lllllll}4 & 3 & 2 & 1 & 1 & 2 & 1 \\ 3 & 6 & 3 & 2 & 1 & 2 & 3 \\ 2 & 3 & 4 & 2 & 1 & 1 & 2 \\ 1 & 2 & 2 & 4 & 2 & 2 & 1 \\ 1 & 1 & 1 & 2 & 4 & 2 & 2 \\ 2 & 2 & 1 & 2 & 2 & 4 & 2 \\ 1 & 3 & 2 & 1 & 2 & 2 & 4\end{array}$

$40 \rightarrow$

voisine = $\begin{array}{lllllll}4 & 2 & 2 & 0 & 1 & 1 & 1 \\ 2 & 4 & 2 & 2 & 1 & 2 & 2 \\ 2 & 2 & 4 & 1 & 1 & 0 & 2 \\ 0 & 2 & 1 & 4 & 2 & 2 & 1 \\ 1 & 1 & 1 & 2 & 4 & 2 & 2 \\ 1 & 2 & 0 & 2 & 2 & 4 & 2 \\ 1 & 2 & 2 & 1 & 2 & 2 & 4\end{array}$

$41 \rightarrow$

Voisine = $\begin{array}{lllllll}4 & 3 & 2 & 0 & 0 & 1 & 1 \\ 3 & 6 & 3 & 2 & 1 & 2 & 3 \\ 2 & 3 & 4 & 1 & 0 & 0 & 2 \\ 0 & 2 & 1 & 4 & 2 & 2 & 1 \\ 0 & 1 & 0 & 2 & 4 & 2 & 2 \\ 1 & 2 & 0 & 2 & 2 & 4 & 2 \\ 1 & 3 & 2 & 1 & 2 & 2 & 4\end{array}$

$42 \rightarrow$

Voisine = $\begin{array}{lllllll}4 & 3 & 2 & 0 & 0 & 1 & 1 \\ 3 & 6 & 3 & 3 & 1 & 3 & 3 \\ 2 & 3 & 4 & 1 & 0 & 0 & 2 \\ 0 & 3 & 1 & 6 & 3 & 4 & 2 \\ 0 & 1 & 0 & 3 & 4 & 3 & 2 \\ 1 & 3 & 0 & 4 & 3 & 6 & 3 \\ 1 & 3 & 2 & 2 & 2 & 3 & 4\end{array}$

$43->$

Voisine $=\begin{array}{lllllll}4 & 3 & 2 & 1 & 1 & 2 & 1 \\ 3 & 6 & 3 & 2 & 0 & 1 & 2 \\ 2 & 3 & 4 & 1 & 0 & 0 & 2 \\ 1 & 2 & 1 & 4 & 2 & 3 & 1 \\ 1 & 0 & 0 & 2 & 4 & 3 & 2 \\ 2 & 1 & 0 & 3 & 3 & 6 & 2 \\ 1 & 2 & 2 & 1 & 2 & 2 & 4\end{array}$

$44 \rightarrow$

Voisine = $\begin{array}{rrrrrrr}4 & 2 & 2 & -1 & 0 & -1 & 0 \\ 2 & 4 & 2 & 0 & -1 & 0 & 1 \\ 2 & 2 & 4 & 1 & 0 & -1 & 1 \\ -1 & 0 & 1 & 4 & 2 & 2 & 1 \\ 0 & -1 & 0 & 2 & 4 & 2 & 2 \\ -1 & 0 & -1 & 2 & 2 & 4 & 2 \\ 0 & 1 & 1 & 1 & 2 & 2 & 4\end{array}$

$12 \times \quad P(7,7)$

$$
S=\begin{array}{rrrrrrr}
1 & 0 & 1 & 0 & 1 & 0 & 1 \\
-1 & -1 & -1 & 0 & 0 & 0 & -1 \\
0 & 0 & -1 & 0 & -1 & 0 & -1 \\
1 & 1 & 1 & 0 & 1 & 0 & 1 \\
-1 & -1 & -1 & -1 & -1 & -1 & -1 \\
0 & 0 & 0 & 0 & -1 & 0 & -1 \\
1 & 1 & 1 & 0 & 1 & 1 & 2
\end{array}
$$

$$
S=\begin{array}{rrrrrrr}
1 & 0 & 1 & 0 & 0 & 0 & 0 \\
-1 & 0 & 0 & 0 & 0 & 0 & 0 \\
0 & 1 & 0 & 0 & 0 & 0 & 1 \\
1 & 0 & 0 & 0 & 0 & 1 & 0 \\
-1 & 0 & 0 & 1 & 0 & 0 & 0 \\
0 & 0 & 0 & 0 & 1 & 0 & 1 \\
1 & 0 & 0 & 0 & 0 & 0 & -1
\end{array}
$$$$
S=\begin{array}{rrrrrrr}
0 & 0 & 1 & 1 & 0 & 0 & 0 \\
-1 & 0 & -1 & 0 & 0 & 0 & 0 \\
0 & -1 & -1 & -1 & 0 & 0 & -1 \\
1 & 1 & 1 & 1 & 0 & 0 & 0 \\
-1 & -1 & -1 & -1 & -1 & -1 & -1 \\
0 & 0 & 0 & -1 & 0 & 0 & 0 \\
1 & 1 & 1 & 1 & 0 & 1 & 1
\end{array}
$$

$$
S=\begin{array}{rrrrrrr}
0 & 0 & 0 & 0 & 1 & 0 & 1 \\
0 & 0 & 0 & 0 & 0 & 1 & 0 \\
-1 & -1 & 0 & 0 & -1 & -1 & -1 \\
0 & 1 & 0 & 0 & 0 & 0 & 1 \\
-1 & -1 & -1 & 0 & 0 & 0 & -1 \\
0 & -1 & 0 & -1 & -1 & -1 & -1 \\
1 & 1 & 0 & 1 & 1 & 1 & 1
\end{array}
$$

$S=\begin{array}{rrrrrrr}1 & 0 & 1 & 0 & 0 & -1 & 0 \\ -1 & 0 & 0 & 0 & 0 & 0 & 0 \\ -1 & -1 & -1 & 0 & 0 & 1 & 0 \\ 1 & 0 & 0 & -1 & 0 & -1 & 0 \\ -1 & 0 & 0 & 1 & 1 & 1 & 1 \\ 0 & 0 & 0 & 0 & 0 & 1 & 0 \\ 1 & 0 & 0 & 0 & 0 & -1 & -1\end{array}$

$$
S=\begin{array}{rrrrrrr}
1 & 0 & 0 & 0 & 0 & 0 & 0 \\
0 & 1 & 1 & 0 & 0 & 0 & 1 \\
-1 & -1 & 0 & 0 & 0 & -1 & -1 \\
1 & 0 & 0 & 0 & 1 & 1 & 1 \\
-1 & 0 & 0 & -1 & -1 & -1 & 0 \\
0 & 0 & 0 & 0 & 0 & -1 & -1 \\
1 & 0 & 0 & 0 & 0 & 1 & 0
\end{array}
$$


$45 \rightarrow$

$24 \times \quad P(7,7)$

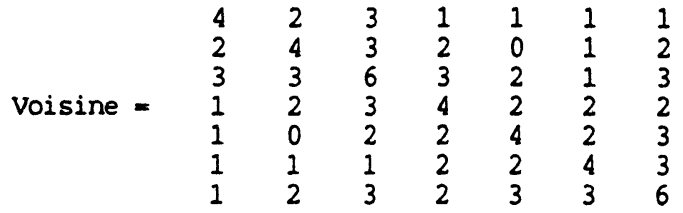

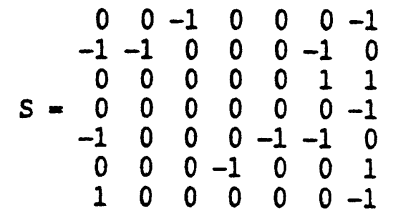

$46 \rightarrow$

$24 \times \quad P(7,7)$

Voisine = $\begin{array}{lllllll}4 & 3 & 3 & 1 & 1 & 1 & 1 \\ 3 & 6 & 4 & 2 & 0 & 0 & 2 \\ 3 & 4 & 6 & 2 & 1 & 0 & 3 \\ 1 & 2 & 2 & 4 & 2 & 2 & 1 \\ 1 & 0 & 1 & 2 & 4 & 2 & 2 \\ 1 & 0 & 0 & 2 & 2 & 4 & 1 \\ 1 & 2 & 3 & 1 & 2 & 1 & 4\end{array}$

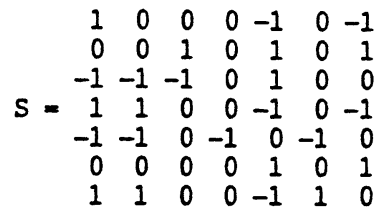

$47 \rightarrow$

$24 \times \quad P(7,7)$

Voisine = $\begin{array}{lllllll}4 & 2 & 2 & 0 & 1 & 1 & 1 \\ 2 & 4 & 2 & 1 & 0 & 0 & 1 \\ 2 & 2 & 4 & 1 & 1 & 0 & 2 \\ 0 & 1 & 1 & 4 & 3 & 2 & 1 \\ 1 & 0 & 1 & 3 & 6 & 3 & 3 \\ 1 & 0 & 0 & 2 & 3 & 4 & 2 \\ 1 & 1 & 2 & 1 & 3 & 2 & 4\end{array}$

$$
S=\begin{array}{rrrrrrr}
0 & 0 & -1 & -1 & -1 & 0 & -1 \\
0 & 0 & 0 & 1 & 1 & 1 & 1 \\
-1 & -1 & 0 & 0 & 0 & -1 & 0 \\
1 & 0 & 0 & -1 & -1 & 0 & -1 \\
0 & 0 & 0 & 0 & 1 & 0 & 1 \\
-1 & -1 & 0 & 1 & 1 & 0 & 0 \\
1 & 1 & 0 & 0 & -1 & 0 & -1
\end{array}
$$

$48 \rightarrow$

$24 \times \quad P(7,7)$

voisine = $\begin{array}{lllllll}4 & 3 & 2 & 0 & 0 & 1 & 1 \\ 3 & 6 & 3 & 1 & 0 & 1 & 3 \\ 2 & 3 & 4 & 2 & 1 & 1 & 2 \\ 0 & 1 & 2 & 4 & 2 & 2 & 1 \\ 0 & 0 & 1 & 2 & 4 & 2 & 2 \\ 1 & 1 & 1 & 2 & 2 & 4 & 2 \\ 1 & 3 & 2 & 1 & 2 & 2 & 4\end{array}$

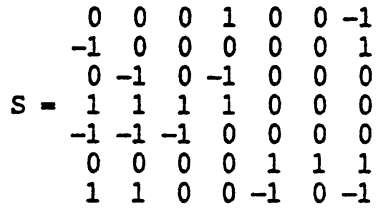

$49 \rightarrow$

$24 \times \quad P(7,7)$

Voisine = $\begin{array}{lllllll}4 & 3 & 2 & 0 & 0 & 1 & 1 \\ 3 & 6 & 3 & 2 & 0 & 2 & 3 \\ 2 & 3 & 4 & 1 & 0 & 0 & 2 \\ 0 & 2 & 1 & 4 & 2 & 3 & 2 \\ 0 & 0 & 0 & 2 & 4 & 3 & 3 \\ 1 & 2 & 0 & 3 & 3 & 6 & 4 \\ 1 & 3 & 2 & 2 & 3 & 4 & 6\end{array}$

$\begin{array}{rrrrrrr}0 & 1 & 1 & 1 & 1 & 0 & 1 \\ -1 & -1 & -1 & 0 & -1 & 0 & -1\end{array}$

$\begin{array}{lllllll}0 & -1 & -1 & -1 & 0 & 0 & -1\end{array}$

$s=\begin{array}{lllllll}1 & 1 & 2 & 1 & 1 & 0 & 1\end{array}$

$\begin{array}{lllllll}-1 & -1 & -1 & 0 & 0 & 1 & 0\end{array}$

$\begin{array}{rrrrrrr}0 & 0 & -1 & -1 & -1 & 0 & -1 \\ 1 & 1 & 1 & 1 & 1 & 0 & 1\end{array}$

$50 \rightarrow \quad 24 \times \quad P(7,7)$

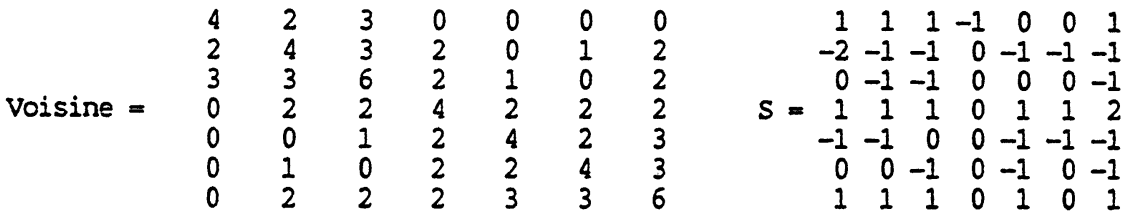


$51 \rightarrow$

$$
\text { Voisine }=\begin{array}{lllllll}
4 & 3 & 3 & 0 & 0 & 0 & 1 \\
3 & 6 & 4 & 3 & 1 & 2 & 3 \\
3 & 4 & 6 & 2 & 1 & 0 & 3 \\
0 & 3 & 2 & 6 & 3 & 3 & 2 \\
0 & 1 & 1 & 3 & 4 & 2 & 2 \\
0 & 2 & 0 & 3 & 2 & 4 & 2 \\
1 & 3 & 3 & 2 & 2 & 2 & 4
\end{array}
$$

$52 \rightarrow \quad 24 \times \quad P(7,7)$

Voisine $=\begin{array}{lllllll}4 & 2 & 2 & 1 & 2 & 2 & 2 \\ 2 & 4 & 2 & 2 & 1 & 1 & 2 \\ 2 & 2 & 4 & 1 & 1 & 0 & 2 \\ 1 & 2 & 1 & 4 & 2 & 2 & 1 \\ 2 & 1 & 1 & 2 & 4 & 2 & 2 \\ 2 & 1 & 0 & 2 & 2 & 4 & 2 \\ 2 & 2 & 2 & 1 & 2 & 2 & 4\end{array}$

$53 \rightarrow$

Voisine = $\begin{array}{lllllll}4 & 3 & 2 & 0 & 1 & 2 & 2 \\ 3 & 6 & 3 & 1 & 0 & 2 & 3 \\ 2 & 3 & 4 & 1 & 0 & 1 & 2 \\ 0 & 1 & 1 & 4 & 2 & 3 & 1 \\ 1 & 0 & 0 & 2 & 4 & 3 & 2 \\ 2 & 2 & 1 & 3 & 3 & 6 & 3 \\ 2 & 3 & 2 & 1 & 2 & 3 & 4\end{array}$

$54 \rightarrow$

Voisine = $\begin{array}{lllllll}4 & 3 & 3 & 0 & 1 & 1 & 2 \\ 3 & 6 & 4 & 2 & 1 & 2 & 4 \\ 3 & 4 & 6 & 2 & 2 & 1 & 4 \\ 0 & 2 & 2 & 4 & 2 & 2 & 2 \\ 1 & 1 & 2 & 2 & 4 & 2 & 3 \\ 1 & 2 & 1 & 2 & 2 & 4 & 3 \\ 2 & 4 & 4 & 2 & 3 & 3 & 6\end{array}$

$55 \rightarrow$

Voisine $=\begin{array}{lllllll}4 & 2 & 2 & 1 & 1 & 2 & 1 \\ 2 & 4 & 2 & 2 & 0 & 2 & 2 \\ 2 & 2 & 4 & 2 & 1 & 1 & 2 \\ 1 & 2 & 2 & 4 & 2 & 3 & 2 \\ 1 & 0 & 1 & 2 & 4 & 3 & 3 \\ 2 & 2 & 1 & 3 & 3 & 6 & 4 \\ 1 & 2 & 2 & 2 & 3 & 4 & 6\end{array}$

$56 \rightarrow$

$6 \times \quad P(7,10)$

Voisine = $\begin{array}{rrrrrrr}4 & 2 & 2 & -1 & 0 & 0 & 1 \\ 2 & 4 & 2 & 0 & -1 & 0 & 1 \\ 2 & 2 & 4 & 0 & 0 & -1 & 2 \\ -1 & 0 & 0 & 4 & 2 & 2 & 0 \\ 0 & -1 & 0 & 2 & 4 & 2 & 2 \\ 0 & 0 & -1 & 2 & 2 & 4 & 1 \\ 1 & 1 & 2 & 0 & 2 & 1 & 4\end{array}$$$
S=\begin{array}{rrrrrrr}
1 & 0 & 0 & 1 & 1 & 1 & 0 \\
-1 & 0 & 0 & 0 & 0 & 0 & 0 \\
-1 & 0 & 0 & 0 & -1 & -1 & 0 \\
1 & 0 & 0 & 0 & 1 & 0 & 0 \\
-1 & -1 & 0 & 0 & 0 & 0 & 0 \\
-1 & 0 & -1 & 0 & -1 & 0 & 0 \\
2 & 1 & 1 & 0 & 1 & 1 & 1
\end{array}
$$$$
S=\begin{array}{rrrrrrr}
1 & 0 & 0 & 0 & 0 & 0 & 0 \\
-1 & 0 & -1 & 0 & 0 & 0 & 0 \\
0 & 1 & 1 & 0 & 0 & 0 & 1 \\
1 & 0 & 0 & 0 & 0 & 1 & 0 \\
-1 & 0 & 0 & 1 & 0 & 0 & 0 \\
-1 & 0 & 0 & -1 & -1 & -1 & 0 \\
1 & 0 & 0 & 0 & 0 & 0 & -1
\end{array}
$$$$
S=\begin{array}{rrrrrrr}
0 & 0 & -1 & -1 & -1 & -1 & -1 \\
-1 & -1 & 0 & 0 & 0 & 0 & 0 \\
0 & 0 & 0 & 1 & 1 & 1 & 1 \\
1 & 0 & 0 & -1 & -1 & -1 & -1 \\
-1 & -1 & 0 & 1 & 1 & 0 & 0 \\
0 & 0 & 0 & 0 & 1 & 1 & 1 \\
1 & 1 & 0 & 0 & -1 & 0 & -1
\end{array}
$$$$
S=\begin{array}{rrrrrrr}
0 & 0 & -1 & 0 & 1 & 1 & 0 \\
-1 & 0 & 1 & 0 & 0 & -1 & 0 \\
0 & 0 & 0 & 0 & -1 & -1 & 0 \\
0 & 0 & -1 & -1 & 0 & 1 & -1 \\
0 & 0 & 0 & 1 & 0 & -1 & 0 \\
0 & -1 & 0 & 0 & 0 & -1 & 0 \\
1 & 0 & 0 & 0 & 0 & 2 & 0
\end{array}
$$$$
\mathbf{S}=\begin{array}{rrrrrrr}
0 & 0 & 1 & 0 & 0 & 1 & 0 \\
-1 & 0 & -1 & -1 & 0 & -1 & -1 \\
0 & 0 & 0 & 0 & -1 & -1 & 0 \\
1 & 0 & 0 & 0 & 0 & 2 & 0 \\
-1 & 1 & 0 & -1 & 0 & -1 & 0 \\
0 & -1 & 0 & 0 & 0 & -1 & 0 \\
1 & 0 & 0 & 1 & 0 & 1 & 0
\end{array}
$$$$
S=\begin{array}{rrrrrrr}
0 & 1 & 0 & 1 & 0 & 0 & 0 \\
0 & 0 & 0 & -1 & 0 & 0 & 0 \\
-1 & -1 & -1 & -1 & 0 & -1 & -1 \\
0 & 1 & 0 & 1 & 0 & 0 & 1 \\
0 & -1 & -1 & -1 & -1 & 0 & -1 \\
0 & 0 & 0 & 0 & 1 & 0 & 0 \\
1 & 1 & 1 & 1 & 0 & 0 & 1
\end{array}
$$ 
$57 \rightarrow$

$\begin{array}{llllllll}4 . & 2 & 2 & 0 & 1 & 1 & 1 \\ 2 & 4 & 2 & 1 & 1 & 1 & 2 \\ 2 & 2 & 4 & 0 & 1 & 0 & 2 \\ 0 & 1 & 0 & 4 & 2 & 2 & 0 \\ 1 & 1 & 1 & 2 & 4 & 2 & 2 \\ 1 & 1 & 0 & 2 & 2 & 4 & 2 \\ 1 & 2 & 2 & 0 & 2 & 2 & 4\end{array}$

$58 \rightarrow$

$$
\text { Voisine = } \begin{array}{lllllll}
4 & 2 & 2 & 0 & 1 & 1 & 1 \\
2 & 4 & 2 & 0 & 0 & 1 & 2 \\
2 & 2 & 4 & 0 & 1 & 0 & 2 \\
0 & 0 & 0 & 4 & 2 & 2 & 0 \\
1 & 0 & 1 & 2 & 4 & 2 & 2 \\
1 & 1 & 0 & 2 & 2 & 4 & 2 \\
1 & 2 & 2 & 0 & 2 & 2 & 4
\end{array}
$$

$59 \rightarrow$

$$
\text { Voisine }=\begin{array}{lllllll}
4 & 3 & 3 & 0 & 1 & 1 & 2 \\
3 & 6 & 4 & 2 & 1 & 1 & 3 \\
3 & 4 & 6 & 1 & 1 & 0 & 3 \\
0 & 2 & 1 & 4 & 2 & 2 & 1 \\
1 & 1 & 1 & 2 & 4 & 2 & 2 \\
1 & 1 & 0 & 2 & 2 & 4 & 2 \\
2 & 3 & 3 & 1 & 2 & 2 & 4
\end{array}
$$$$
60 \rightarrow
$$$$
12 \times \quad P(7,11)
$$$$
\text { voisine }=\begin{array}{rrrrrrr}
16 / 3 & 8 / 3 & 8 / 3 & 0 & 4 / 3 & 2 & 4 / 3 \\
8 / 3 & 4 & 2 & 2 / 3 & 0 & 4 / 3 & 2 \\
8 / 3 & 2 & 4 & 0 & 2 \beta & 0 & 2 \\
0 & 2 / 3 & 0 & 4 & 2 & 8 / 3 & 2 / 3 \\
4 / 3 & 0 & 2 / 3 & 2 & 4 & 8 / 3 & 2 \\
2 & 4 / 3 & 0 & 8 / 3 & 8 / 3 & 16 / 3 & 2 \\
4 / 3 & 2 & 2 & 2 / 3 & 2 & 2 & 4
\end{array}
$$$$
61 \rightarrow
$$

$$
24 \times \quad P(7,11)
$$

$$
\text { voisine }=\begin{array}{rrrrrrr}
4 & 8 / 3 & 2 & 0 & 0 & 4 / 3 & 2 / 3 \\
8 / 3 & 16 / 3 & 8 / 3 & 4 \beta & 0 & 4 / 3 & 8 / 3 \\
2 & 8 / 3 & 4 & 4 / 3 & 2 / 3 & 2 / 3 & 2 \\
0 & 4 / 3 & 4 \beta & 4 & 2 & 2 & 4 / 3 \\
0 & 0 & 2 \beta & 2 & 4 & 2 & 2 \\
4 / 3 & 4 / 3 & 2 / 3 & 2 & 2 & 4 & 2 \\
2 / 3 & 8 / 3 & 2 & 4 / 3 & 2 & 2 & 4
\end{array}
$$$$
62 \rightarrow \quad 24 \times \quad P(7,11)
$$

$$
\text { Voisine }=\begin{array}{rrrrrrr}
4 & 8 / 3 & 2 & 0 & 0 & 2 / 3 & 2 / 3 \\
8 / 3 & 16 / 3 & 8 / \beta & 2 & 0 & 4 / 3 & 2 \\
2 & 8 / 3 & 4 & 4 / 3 & 2 / 3 & 0 & 2 \\
0 & 2 & 4 / 3 & 4 & 2 & 2 & 4 / 3 \\
0 & 0 & 2 / 3 & 2 & 4 & 2 & 8 / 3 \\
2 / 3 & 4 / 3 & 0 & 2 & 2 & 4 & 8 / 3 \\
2 \beta & 2 & 2 & 4 / 3 & 8 / 3 & 8 / 3 & 16 / 3
\end{array}
$$

$$
S=\begin{array}{rrrrrrr}
0 & -1 & 0 & 0 & 0 & 0 & 0 \\
0 & 0 & 0 & 0 & 0 & 1 & 0 \\
0 & 1 & 0 & 1 & 0 & 0 & 0 \\
0 & 0 & 1 & 0 & 1 & 0 & 1 \\
0 & 0 & -1 & 0 & -1 & 0 & 0 \\
0 & 0 & 0 & 0 & -1 & 0 & -1 \\
1 & 0 & 1 & 0 & 1 & 0 & 1
\end{array}
$$

$$
S=\begin{array}{rrrrrrr}
0 & -1 & 0 & 0 & 0 & 1 & 0 \\
0 & 0 & 0 & -1 & 0 & -1 & 0 \\
0 & 1 & 0 & 0 & 0 & 0 & 0 \\
1 & 0 & 0 & 1 & 0 & 1 & 1 \\
-1 & 0 & 0 & -1 & 0 & -1 & 0 \\
0 & 1 & 1 & 0 & 1 & 0 & 0 \\
1 & -1 & 0 & 1 & -1 & 1 & 0
\end{array}
$$

$$
S=\begin{array}{rrrrrrr}
0 & -1 & -1 & -1 & -1 & -1 & -1 \\
0 & 1 & 1 & 0 & 1 & 0 & 1 \\
0 & 1 & 0 & 1 & 0 & 0 & 0 \\
0 & -1 & -1 & -1 & -1 & 0 & -1 \\
0 & 0 & 1 & 0 & 0 & 0 & 0 \\
0 & 1 & 0 & 1 & 0 & 0 & 1 \\
1 & -1 & 0 & 0 & 0 & 1 & 0
\end{array}
$$

$$
S=\begin{array}{rrrrrrr}
0 & 0 & 0 & 1 & -1 & 1 & 0 \\
0 & 1 & 0 & -1 & 1 & -1 & 0 \\
0 & -1 & 0 & -1 & 0 & -1 & 0 \\
1 & 0 & 1 & 1 & 0 & 1 & 0 \\
0 & 0 & -1 & -1 & 1 & 0 & 1 \\
0 & 0 & 0 & 0 & 0 & -1 & 0 \\
0 & 0 & 0 & 1 & -1 & 1 & -1
\end{array}
$$

$$
S=\begin{array}{rrrrrrr}
0 & 0 & 0 & 1 & -1 & 1 & 0 \\
-1 & 0 & -1 & -1 & 0 & -1 & 0 \\
0 & 0 & 0 & -1 & 1 & 0 & 0 \\
1 & 0 & 0 & 1 & 0 & 1 & 1 \\
-1 & 0 & 0 & -1 & 0 & -1 & 0 \\
0 & 0 & 0 & -1 & 0 & -1 & -1 \\
1 & 1 & 1 & 2 & 0 & 1 & 0
\end{array}
$$

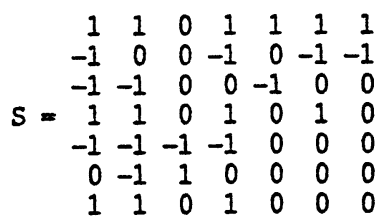


$63->$

$$
\text { Voisine = } \begin{array}{rrrrrrr}
4 & 8 / 3 & 2 & 0 & 2 / 3 & 4 / 3 & 4 / 3 \\
8 / 3 & 16 / 3 & 8 / 3 & 2 & 2 / 3 & 2 & 8 / 3 \\
2 & 8 / 3 & 4 & 2 / 3 & 0 & 0 & 2 \\
0 & 2 & 2 / 3 & 4 & 2 & 8 / 3 & 4 / 3 \\
2 / 3 & 2 / 3 & 0 & 2 & 4 & 8 / 3 & 2 \\
4 / 3 & 2 & 0 & 8 / 3 & 8 / 3 & 16 / 3 & 8 / 3 \\
4 / 3 & 8 / 3 & 2 & 4 / 3 & 2 & 8 / 3 & 4
\end{array}
$$$$
64 \rightarrow
$$$$
24 \times \quad P(7,11)
$$
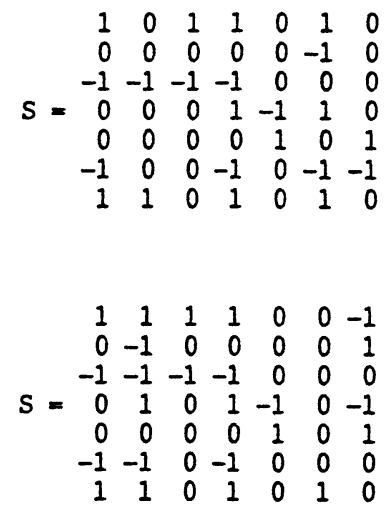

$65 \rightarrow$

$6 \times \quad P(7,14)$

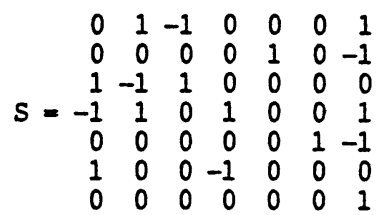

$66 \rightarrow$

$12 \times \quad P(7,14)$

$$
\text { Voisine }=\begin{array}{rrrrrrr}
4 & 8 / 3 & 2 & 2 / 3 & 4 / 3 & 2 & 4 / 3 \\
8 / 3 & 16 / 3 & 8 / 3 & 2 & 2 / 3 & 2 & 8 / 3 \\
2 & 8 / 3 & 4 & 4 / 3 & 2 / 3 & 2 / 3 & 2 \\
2 / 3 & 2 & 4 / 3 & 4 & 2 & 8 / 3 & 4 / 3 \\
4 / 3 & 2 / 3 & 2 / 3 & 2 & 4 & 8 / 3 & 2 \\
2 & 2 & 2 / 3 & 8 / 3 & 8 / 3 & 16 / 3 & 8 / 3 \\
4 / 3 & 8 / 3 & 2 & 4 / 3 & 2 & 8 / 3 & 4
\end{array}
$$

$67 \rightarrow$

$24 \times \quad P(7,14)$

Voisine $=\begin{array}{rrrrrrr}16 / 3 & 8 / 3 & 8 / 3 & 0 & 2 & 2 & 2 \\ 8 / 3 & 4 & 2 & 2 / 3 & 2 / 3 & 4 / 3 & 2 \\ 8 / 3 & 2 & 4 & 0 & 4 / 3 & 0 & 2 \\ 0 & 2 / 3 & 0 & 4 & 8 / 3 & 8 / 3 & 2 / 3 \\ 2 & 2 / 3 & 4 / 3 & 8 / 3 & 16 / 3 & 10 / 3 & 8 / 3 \\ 2 & 4 / 3 & 0 & 8 / 3 & 10 / 3 & 16 / 3 & 8 / 3 \\ 2 & 2 & 2 & 2 / 3 & 8 / 3 & 8 / 3 & 4\end{array}$

$68 \rightarrow$

$24 \times \quad P(7,14)$

voisine $=\begin{array}{rrrrrrr}4 & 2 & 2 & 0 & 4 / 3 & 4 / 3 & 4 / 3 \\ 2 & 4 & 2 & 2 \beta & 0 & 2 \beta & 2 \\ 2 & 2 & 4 & 0 & 2 \beta & -2 / 3 & 2 \\ 0 & 2 \beta & 0 & 4 & 2 & 2 & 0 \\ 4 / 3 & 0 & 2 / 3 & 2 & 4 & 2 & 4 \beta \\ 4 / 3 & 2 / 3 & -2 / 3 & 2 & 2 & 4 & 4 / 3 \\ 4 / 3 & 2 & 2 & 0 & 4 / 3 & 4 / 3 & 4\end{array}$

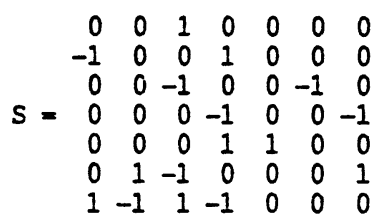

$S=\begin{array}{rrrrrrr}0 & 0 & 0 & 0 & 0 & -1 & 0 \\ 0 & -1 & 0 & 0 & 0 & 0 & -1 \\ 0 & 0 & 1 & 1 & 0 & 1 & 1 \\ -1 & 0 & 0 & 0 & -1 & -1 & 0 \\ 0 & 0 & 0 & 0 & 0 & 1 & -1 \\ 1 & 0 & 0 & 0 & 0 & 1 & 1 \\ 0 & 0 & 0 & -1 & 0 & -1 & 0\end{array}$

$S=\begin{array}{rrrrrrr}1 & -1 & 1 & 0 & 0 & 0 & 0 \\ -1 & 0 & 0 & 0 & 0 & 0 & -1 \\ -1 & 1 & -1 & 0 & -1 & 0 & 0 \\ 1 & 0 & 1 & 0 & 0 & 1 & 1 \\ 0 & 0 & -1 & -1 & 0 & 0 & -1 \\ -1 & 1 & -1 & 0 & 0 & -1 & 0 \\ 1 & -1 & 1 & 0 & 0 & 0 & 1\end{array}$ 
$69 \rightarrow$

$24 \times \quad P(7,14)$

Voisine $=\begin{array}{rrrrrrr}4 & 8 / 3 & 2 & 2 / 3 & 4 / 3 & 2 & 4 / 3 \\ 8 / 3 & 16 / 3 & 8 / 3 & 4 / 3 & 0 & 4 / 3 & 8 / 3 \\ 2 & 8 / 3 & 4 & 2 / 3 & 0 & 0 & 2 \\ 2 / 3 & 4 / 3 & 2 / 3 & 4 & 2 & 8 / 3 & 2 / 3 \\ 4 / 3 & 0 & 0 & 2 & 4 & 8 / 3 & 4 / 3 \\ 2 & 4 / 3 & 0 & 8 / 3 & 8 / 3 & 16 / 3 & 2 \\ 4 / 3 & 8 / 3 & 2 & 2 / 3 & 4 / 3 & 2 & 4\end{array}$

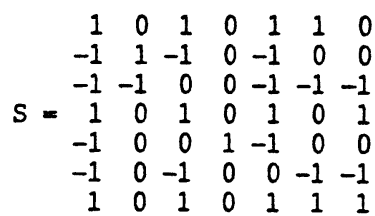

$70 \rightarrow$

$12 \times \quad P(7,15)$

Voisine $=\begin{array}{rrrrrrr}4 & 5 / 2 & 2 & 1 / 2 & 1 & 3 / 2 & 1 \\ 5 / 2 & 5 & 5 / 2 & 3 / 2 & 0 & 3 / 2 & 5 / 2 \\ 2 & 5 / 2 & 4 & 1 & 0 & 0 & 3 / 2 \\ 1 / 2 & 3 / 2 & 1 & 4 & 2 & 5 / 2 & 1 \\ 1 & 0 & 0 & 2 & 4 & 5 / 2 & 3 / 2 \\ 3 / 2 & 3 / 2 & 0 & 5 / 2 & 5 / 2 & 5 & 5 / 2 \\ 1 & 5 / 2 & 3 / 2 & 1 & 3 / 2 & 5 / 2 & 4\end{array}$

$\begin{array}{rrrrrrr}0 & -1 & 0 & 1 & 0 & -1 & 1 \\ -1 & 1 & 0 & -1 & 0 & 0 & 0\end{array}$

$\begin{array}{lllllll}0 & 1 & -1 & 0 & 0 & 1 & -1\end{array}$

$S=\begin{array}{rrrrrrr}1 & -1 & 0 & 1 & 0 & -1 & 0\end{array}$

$\begin{array}{rrrrrrr}-1 & 1 & 0 & 0 & -1 & 1 & 0\end{array}$

$\begin{array}{rrrrrrr}0 & 0 & 0 & -1 & 0 & 1 & -1 \\ 1 & -1 & 0 & 1 & 0 & -1 & 1\end{array}$

$71 \rightarrow$

$2 \times \quad P(7,16)$

$\begin{array}{rrrrrrr}4 & 2 & 2 & -2 / 3 & 2 / 3 & 0 & 2 / 3 \\ 2 & 4 & 2 & 0 & -2 / 3 & 2 / 3 & 2 \\ 2 & 2 & 4 & 2 / 3 & 0 & -2 / 3 & 4 / 3 \\ \text { Voisine }=-2 / 3 & 0 & 2 / 3 & 4 & 2 & 2 & 2 / 3 \\ 2 / 3 & -2 / 3 & 0 & 2 & 4 & 2 & 4 / 3 \\ 0 & 2 / 3 & -2 / 3 & 2 & 2 & 4 & 2 \\ 2 / 3 & 2 & 4 / 3 & 2 / 3 & 4 / 3 & 2 & 4\end{array}$

$\begin{array}{lllllll}0 & 1 & 0 & 1 & 1 & 0 & 1\end{array}$

$\begin{array}{lllllll}0 & -1 & 0 & -1 & 0 & -1 & -1\end{array}$

$\begin{array}{lllllll}0 & -1 & -1 & 0 & -1 & 0 & -1\end{array}$

$S=\begin{array}{lllllll}0 & 1 & 0 & 0 & 1 & 0 & 1\end{array}$

$\begin{array}{lllllll}0 & -1 & 0 & -1 & 0 & 0 & -1\end{array}$

$\begin{array}{rrrrrrr}0 & 0 & -1 & 0 & -1 & 0 & -1 \\ 1 & 1 & 1 & 1 & 1 & 1 & 2\end{array}$

$72 \rightarrow$

$12 \times \quad P(7,16)$

Voisine $=\begin{array}{rrrrrrr}4 & 2 & 8 / 3 & 0 & 2 / 3 & 2 / 3 & 2 / 3 \\ 2 & 4 & 8 / 3 & 4 / 3 & 0 & 2 / 3 & 2 \\ 8 / 3 & 8 / 3 & 16 / 3 & 4 / 3 & 4 / 3 & 0 & 8 / 3 \\ 0 & 4 / 3 & 4 / 3 & 16 / 3 & 8 / 3 & 8 / 3 & 4 / 3 \\ 2 / 3 & 0 & 4 / 3 & 8 / 3 & 4 & 2 & 2 \\ 2 / 3 & 2 / 3 & 0 & 8 / 3 & 2 & 4 & 4 / 3 \\ 2 / 3 & 2 & 8 / 3 & 4 / 3 & 2 & 4 / 3 & 4\end{array}$

$73 \rightarrow$

$24 \times \quad P(7,17)$

$\begin{array}{rrrrrrrr}4 & 2 & 2 & 2 \beta & 4 / 3 & 2 & 4 / 3 \\ 2 & 4 & 2 & 4 / 3 & 0 & 2 & 8 / 3 \\ 2 & 2 & 4 & 4 / 3 & 2 / 3 & 2 / 3 & 2 \\ \text { Voisine }= & 2 / 3 & 4 / 3 & 4 / 3 & 4 & 2 & 8 / 3 & 4 / 3 \\ 4 / 3 & 0 & 2 / 3 & 2 & 4 & 8 / 3 & 2 \\ 2 & 2 & 2 / 3 & 8 / 3 & 8 / 3 & 16 / 3 & 10 / 3 \\ 4 / 3 & 8 / 3 & 2 & 4 / 3 & 2 & 10 / 3 & 16 / 3\end{array}$

$74 \rightarrow \quad 24 \times \quad P(7,17)$

Voisine $=\begin{array}{rrrrrrr}4 & 2 & 2 & 2 / 3 & 4 / 3 & 4 / 3 & 4 / 3 \\ 2 & 4 & 2 & 2 & 2 / 3 & 4 / 3 & 2 \\ 2 & 2 & 4 & 4 / 3 & 2 / 3 & 0 & 2 \\ 2 / 3 & 2 & 4 / 3 & 4 & 2 & 2 & 4 / 3 \\ 4 / 3 & 2 / 3 & 2 / 3 & 2 & 4 & 2 & 2 \\ 4 / 3 & 4 / 3 & 0 & 2 & 2 & 4 & 2 \\ 4 / 3 & 2 & 2 & 4 / 3 & 2 & 2 & 4\end{array}$
$S=\begin{array}{rrrrrrr}0 & 0 & 0 & 0 & 0 & -1 & 0 \\ -1 & 0 & -1 & 0 & -1 & 0 & 0 \\ 0 & 0 & 1 & 1 & 1 & 1 & 0 \\ 0 & 1 & 0 & 0 & 0 & 0 & 0 \\ 0 & 0 & 0 & 0 & -1 & 0 & 1 \\ 0 & 0 & 0 & 0 & 1 & 0 & 0 \\ 1 & 0 & 0 & 0 & 0 & 0 & 0\end{array}$

$\begin{array}{lllllll}1 & 0 & -1 & 0 & 0 & 1 & 0\end{array}$

$\mathbf{S}=\begin{array}{rrrrrrr}0 & 1 & 1 & 1 & 0 & 0 & 1 \\ -1 & 0 & 0 & -1 & 0 & -1 & -1 \\ 0 & -1 & -1 & 0 & -1 & 0 & -1\end{array}$

$\begin{array}{rrrrrrr}0 & 1 & 1 & 0 & 0 & 0 & 1\end{array}$ $\begin{array}{rrrrrrr}0 & 0 & 0 & 0 & 1 & -1 & 0 \\ 1 & -1 & 0 & 0 & 0 & 1 & 0\end{array}$ 
$75->$

$24 \times \quad P(7,18)$
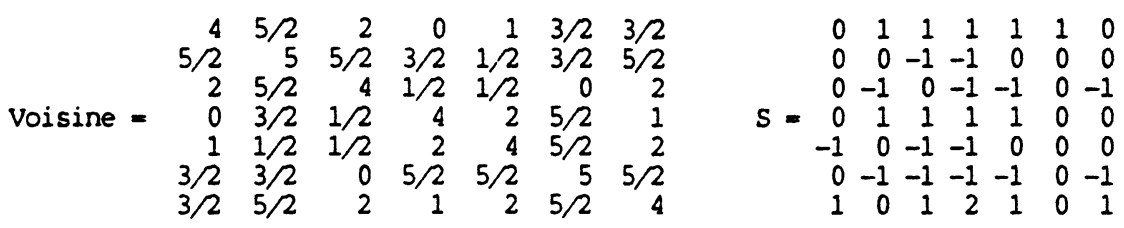

$76 \rightarrow$

$12 \times \quad P(7,20)$
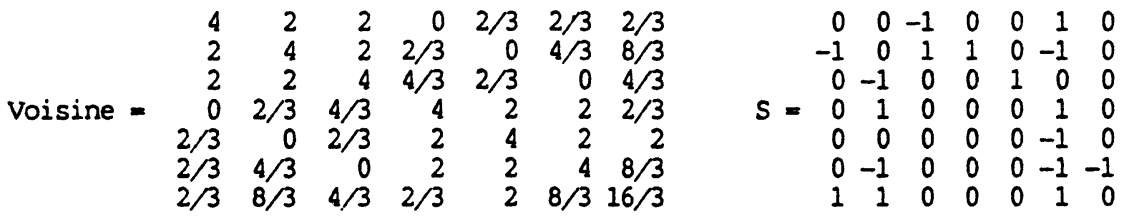

$77 \rightarrow$

$12 \times \quad P(7,21)$
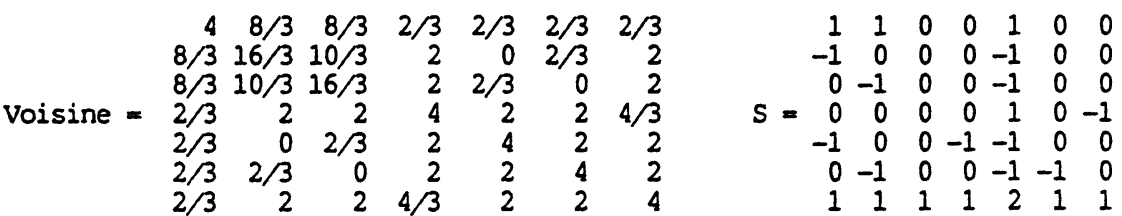

$78 \rightarrow$

$12 \times \quad P(7,22)$

Voisine = $\begin{array}{lllllll}4 & 3 & 3 & 1 & 1 & 1 & 1 \\ 3 & 6 & 4 & 3 & 1 & 2 & 3 \\ 3 & 4 & 6 & 3 & 2 & 1 & 3 \\ 1 & 3 & 3 & 6 & 3 & 3 & 2 \\ 1 & 1 & 2 & 3 & 4 & 2 & 2 \\ 1 & 2 & 1 & 3 & 2 & 4 & 2 \\ 1 & 3 & 3 & 2 & 2 & 2 & 4\end{array}$

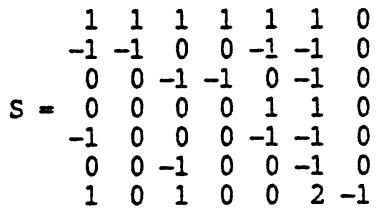

$79 \rightarrow$

$12 \times \quad P(7,22)$

Voisine $=\begin{array}{rrrrrrr}4 & 2 & 2 & -1 & 0 & 0 & 0 \\ 2 & 4 & 2 & 1 & 0 & 1 & 2 \\ 2 & 2 & 4 & 1 & 1 & 0 & 2 \\ -1 & 1 & 1 & 4 & 2 & 2 & 2 \\ 0 & 0 & 1 & 2 & 4 & 2 & 3 \\ 0 & 1 & 0 & 2 & 2 & 4 & 3 \\ 0 & 2 & 2 & 2 & 3 & 3 & 6\end{array}$

$S=\begin{array}{rrrrrrr}1 & 1 & 1 & 1 & 1 & 0 & 1 \\ -1 & -1 & 0 & 0 & -1 & 0 & -1 \\ 0 & 0 & -1 & -1 & 0 & 0 & -1 \\ 1 & 1 & 1 & 1 & 1 & 1 & 1 \\ -1 & -1 & 0 & -1 & -1 & 0 & -1 \\ 0 & -1 & -1 & -1 & 0 & 0 & -1 \\ 1 & 1 & 1 & 1 & 0 & 0 & 1\end{array}$

$80 \rightarrow$

$24 \times \quad P(7,22)$

Voisine $=\begin{array}{lllllll}6 & 3 & 3 & 0 & 2 & 2 & 2 \\ 3 & 4 & 2 & 1 & 1 & 2 & 2 \\ 3 & 2 & 4 & 0 & 1 & 0 & 2 \\ 0 & 1 & 0 & 4 & 3 & 3 & 1 \\ 2 & 1 & 1 & 3 & 6 & 4 & 3 \\ 2 & 2 & 0 & 3 & 4 & 6 & 3 \\ 2 & 2 & 2 & 1 & 3 & 3 & 4\end{array}$

$S=\begin{array}{rrrrrrr}0 & 0 & 1 & 1 & 0 & 0 & 0 \\ 0 & 0 & -1 & -1 & 1 & 0 & 0 \\ 0 & 0 & 0 & -1 & -1 & 0 & 0 \\ 1 & 0 & 1 & 1 & 0 & 0 & 0 \\ -1 & -1 & -1 & -1 & 0 & 0 & 0 \\ 0 & 1 & 0 & 0 & 0 & 0 & 1 \\ 1 & 0 & 1 & 1 & 0 & 1 & -1\end{array}$ 
$81 \rightarrow$

$12 \times \quad P(7,23)$

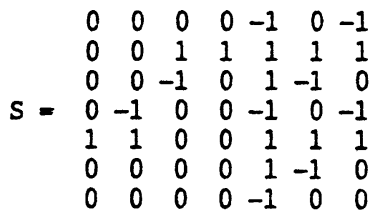

$82 \rightarrow$

$24 \times \quad P(7,23)$

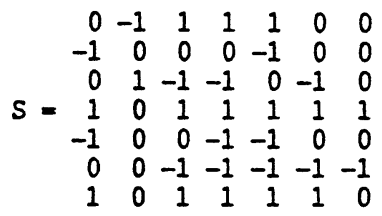

$\begin{array}{rrrrrrr}0 & -1 & 1 & 1 & 1 & 0 & 0 \\ -1 & 0 & 0 & 0 & -1 & 0 & 0\end{array}$

$\begin{array}{lllllll}0 & 1 & -1 & -1 & 0 & -1 & 0\end{array}$ $\begin{array}{rrrrrrr}1 & 0 & 1 & 1 & 1 & 1 & 1 \\ -1 & 0 & 0 & -1 & -1 & 0 & 0\end{array}$ $\begin{array}{rrrrrrr}0 & 0 & -1 & -1 & -1 & -1 & -1 \\ 1 & 0 & 1 & 1 & 1 & 1 & 0\end{array}$

$83 \rightarrow$

$24 \times \quad P(7,24)$
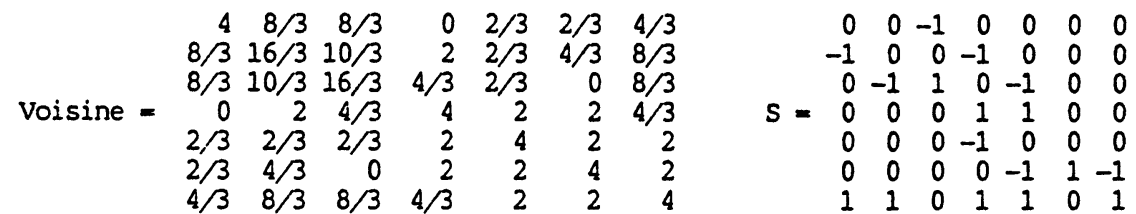

$84 \rightarrow$

$24 \times \quad P(7,24)$
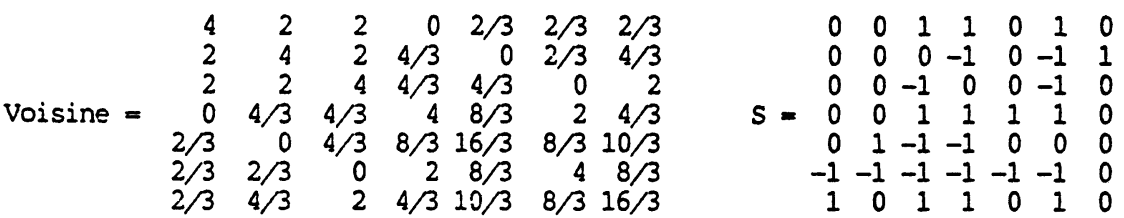

$85 \rightarrow$

$24 \times \quad P(7,24)$

Voisine $=\begin{array}{rrrrrrr}4 & 8 / 3 & 2 & 0 & 2 / 3 & 4 / 3 & 4 / 3 \\ 8 / 3 & 16 / 3 & 8 / 3 & 4 / 3 & 2 / 3 & 4 / 3 & 8 / 3 \\ 2 & 8 / 3 & 4 & 2 / 3 & 2 / 3 & 0 & 2 \\ 0 & 4 / 3 & 2 / 3 & 4 & 2 & 2 & 2 / 3 \\ 2 / 3 & 2 / 3 & 2 / 3 & 2 & 4 & 2 & 2 \\ 4 / 3 & 4 / 3 & 0 & 2 & 2 & 4 & 2 \\ 4 / 3 & 8 / 3 & 2 & 2 \beta & 2 & 2 & 4\end{array}$

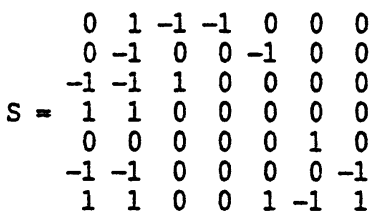

$86 \rightarrow$

$12 \times \quad P(7,25)$

$\begin{array}{lllllll}4 & 8 / 3 & 8 / 3 & 2 / 3 & 4 / 3 & 4 / 3 & 4 / 3\end{array}$ $\begin{array}{llllllll}8 / 3 & 16 / 3 & 10 / 3 & 4 / 3 & 0 & 2 / 3 & 8 / 3\end{array}$ $\begin{array}{llllllll}8 / 3 & 10 / 3 & 16 / 3 & 4 / 3 & 2 / 3 & 0 & 8 / 3\end{array}$ Voisine $=\begin{array}{rrrrrrr}2 / 3 & 4 / 3 & 4 / 3 & 4 & 2 & 2 & 2 / 3 \\ 4 / 3 & 0 & 2 / 3 & 2 & 4 & 2 & 4 / 3\end{array}$

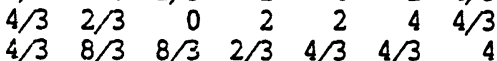

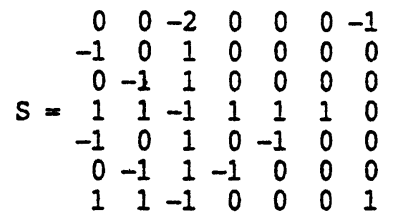




$$
87 \rightarrow
$$

$24 \times \quad P(7,25)$
$88 \rightarrow$

$$
\text { Voisine }=\begin{array}{rrrrrrr}
16 / 3 & 8 / 3 & 10 / 3 & 0 & 2 & 4 / 3 & 2 \\
8 / 3 & 4 & 8 / 3 & 2 / 3 & 2 / 3 & 2 / 3 & 2 \\
10 / 3 & 8 / 3 & 16 / 3 & 2 / 3 & 2 & 0 & 8 / 3 \\
0 & 2 / 3 & 2 / 3 & 4 & 8 / 3 & 2 & 2 / 3 \\
2 & 2 / 3 & 2 & 8 / 3 & 16 / 3 & 8 / 3 & 8 / 3 \\
4 / 3 & 2 / 3 & 0 & 2 & 8 / 3 & 4 & 2 \\
2 & 2 & 8 / 3 & 2 / 3 & 8 / 3 & 2 & 4
\end{array}
$$

$$
X(7,25)
$$$$
\text { Voisine = } \begin{array}{rrrrrrr}
4 & 8 / 3 & 2 & 0 & 2 / 3 & 4 / 3 & 4 / 3 \\
8 / 3 & 16 / 3 & 8 / 3 & 4 / 3 & 0 & 4 / 3 & 8 / 3 \\
2 & 8 / 3 & 4 & 4 / 3 & 2 / 3 & 2 / 3 & 2 \\
0 & 4 / 3 & 4 / 3 & 4 & 2 & 8 / 3 & 4 / 3 \\
2 / 3 & 0 & 2 / 3 & 2 & 4 & 8 / 3 & 2 \\
4 / 3 & 4 / 3 & 2 / 3 & 8 / 3 & 8 / 3 & 16 / 3 & 8 / 3 \\
4 / 3 & 8 / 3 & 2 & 4 / 3 & 2 & 8 / 3 & 4
\end{array}
$$

$$
\mathbf{S}=\begin{array}{rrrrrrr}
0 & 0 & 0 & 1 & 1 & 1 & -1 \\
0 & -1 & 0 & -1 & -1 & -1 & 0 \\
0 & 1 & 0 & 0 & -1 & 0 & 1 \\
0 & 0 & 1 & 1 & 1 & 1 & 0 \\
-1 & -1 & -1 & -1 & -1 & -1 & -1 \\
0 & 1 & 0 & 0 & -1 & -1 & 1 \\
1 & 0 & 0 & 1 & 2 & 1 & 0
\end{array}
$$

$$
S=\begin{array}{rrrrrrr}
0 & 0 & -1 & 1 & 1 & 1 & -1 \\
0 & 0 & 1 & -1 & -1 & 0 & 1 \\
0 & 0 & 1 & 0 & 0 & 0 & 0 \\
0 & -1 & -1 & 0 & 1 & 0 & -1 \\
0 & 0 & 0 & -1 & -1 & 0 & 0 \\
0 & 1 & 1 & 0 & 0 & 0 & 1 \\
1 & 0 & -1 & 1 & 1 & 0 & 0
\end{array}
$$

$89 \rightarrow$

$12 \times \quad P(7,27)$

$$
\text { Voisine }=\quad \begin{array}{lllllll}
6 & 3 & 4 & 0 & 2 & 1 & 2 \\
3 & 4 & 3 & 1 & 1 & 1 & 2 \\
4 & 3 & 6 & 1 & 2 & 0 & 3 \\
0 & 1 & 1 & 4 & 3 & 2 & 1 \\
2 & 1 & 2 & 3 & 6 & 3 & 3 \\
1 & 1 & 0 & 2 & 3 & 4 & 2 \\
2 & 2 & 3 & 1 & 3 & 2 & 4
\end{array}
$$

$90 \rightarrow$

$2 \times \quad P(7,28)$

$$
\begin{array}{rrrrrrr}
4 & 2 & 2 & -2 & -1 & -1 & 1 \\
2 & 4 & 2 & -1 & -2 & -1 & 2 \\
2 & 2 & 4 & -1 & -1 & -2 & 2 \\
-2 & -1 & -1 & 4 & 2 & 2 & -1 \\
-1 & -2 & -1 & 2 & 4 & 2 & 0 \\
-1 & -1 & -2 & 2 & 2 & 4 & 0 \\
1 & 2 & 2 & -1 & 0 & 0 & 4
\end{array}
$$

$91 \rightarrow$

$$
12 \times \quad P(7,28)
$$

$$
\text { Voisine = } \begin{array}{lllllll}
4 & 2 & 2 & 0 & 1 & 1 & 1 \\
2 & 4 & 2 & 1 & 0 & 1 & 2 \\
2 & 2 & 4 & 1 & 1 & 0 & 2 \\
0 & 1 & 1 & 6 & 3 & 4 & 1 \\
1 & 0 & 1 & 3 & 4 & 3 & 2 \\
1 & 1 & 0 & 4 & 3 & 6 & 2 \\
1 & 2 & 2 & 1 & 2 & 2 & 4
\end{array}
$$

$92 \rightarrow$

$$
12 \times \quad P(7,28)
$$

$$
\text { Voisine = } \begin{array}{lllllll}
6 & 4 & 4 & 0 & 1 & 1 & 2 \\
4 & 6 & 4 & 2 & 1 & 2 & 3 \\
4 & 4 & 6 & 1 & 1 & 0 & 3 \\
0 & 2 & 1 & 4 & 2 & 2 & 1 \\
1 & 1 & 1 & 2 & 4 & 2 & 2 \\
1 & 2 & 0 & 2 & 2 & 4 & 2 \\
2 & 3 & 3 & 1 & 2 & 2 & 4
\end{array}
$$

$$
S=\begin{array}{rrrrrrr}
0 & 0 & 0 & 0 & 0 & -1 & 0 \\
0 & -1 & 0 & 0 & 0 & 0 & -1 \\
0 & 0 & 0 & -1 & 0 & 0 & -1 \\
0 & 0 & 1 & 0 & 0 & 0 & 1 \\
0 & 0 & 0 & 0 & 1 & 0 & 0 \\
1 & 0 & 0 & 0 & 0 & 0 & 0 \\
0 & 0 & 0 & 0 & 0 & 0 & 1
\end{array}
$$

$\begin{array}{rrrrrrr}1 & 1 & 0 & 1 & 1 & 1 & 2 \\ -1 & -1 & 0 & -1 & 0 & 1 & 2\end{array}$

$\begin{array}{rrrrrrr}0 & 0 & 1 & 0 & 0 & 0 & 1\end{array}$

$S=\begin{array}{lllllll}1 & 0 & 0 & 1 & 0 & 1 & 1\end{array}$ $\begin{array}{lllllll}-1 & -1 & 0 & -2 & 0 & -1 & -2\end{array}$ $\begin{array}{rrrrrrr}0 & 0 & 0 & 0 & 0 & -1 & 0 \\ 1 & 1 & 0 & 1 & 0 & 1 & 1\end{array}$

$$
S=\begin{array}{rrrrrrr}
1 & 0 & 1 & 0 & 0 & 0 & 1 \\
-1 & 0 & 0 & 0 & 0 & 0 & -1 \\
-1 & 0 & -1 & -1 & 0 & 0 & -1 \\
1 & 0 & 0 & 0 & 0 & -1 & 0 \\
-1 & 0 & 0 & 0 & 0 & 0 & 0 \\
-1 & -1 & -1 & -1 & -1 & 0 & -2 \\
2 & 0 & 1 & 1 & 1 & 0 & 2
\end{array}
$$


$93 \rightarrow \quad 12 \times \quad P(7,28)$

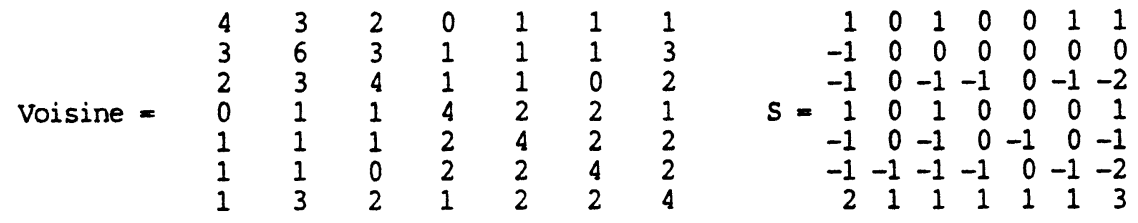

$94 \rightarrow \quad 12 \times \quad P(7,31)$

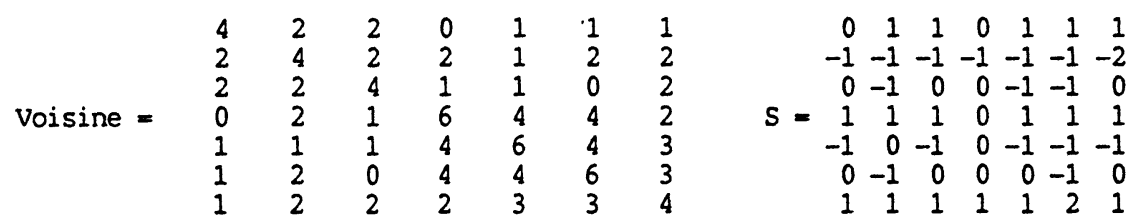

Nombre total de voisines : 1482

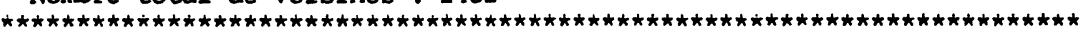

Forme parfaite $P(7,8)$.

$\begin{array}{rrrrrrr}4 & -1 & -1 & 2 & -1 & 2 & 0 \\ -1 & 4 & 1 & 0 & 0 & 0 & 2 \\ -1 & 1 & 4 & 0 & 0 & 0 & 2 \\ 2 & 0 & 0 & 4 & 1 & 0 & 2 \\ -1 & 0 & 0 & 1 & 4 & 0 & 2 \\ 2 & 0 & 0 & 0 & 0 & 4 & 0 \\ 0 & 2 & 2 & 2 & 2 & 0 & 4\end{array}$

Nombre de vecteurs minimaux $\quad: 32$

Minimum

4

Déterminant

Déterminant (minimum normalisé à 2) : $9 / 2$

Ordre du groupe des automorphismes : 128

Liste des orbites des voisines de la forme $P(7,8)$ :

$\begin{array}{rrr}1 \rightarrow & 4 \times & P(7,1) \\ 2 \rightarrow & 4 \times & P(7,1) \\ 3 \rightarrow & 8 \times & P(7,1) \\ 4 \rightarrow & 8 \times & P(7,1) \\ 5 \rightarrow & 16 \times & P(7,1) \\ 6 \rightarrow & 16 \times & P(7,1) \\ 7 \rightarrow & 16 \times & P(7,1) \\ 8 \rightarrow & 16 \times & P(7,1) \\ 9 \rightarrow & 16 \times & P(7,1) \\ 10 \rightarrow & 32 \times & P(7,1) \\ 11 \rightarrow & 8 \times & P(7,3) \\ 12 \rightarrow & 16 \times & P(7,3) \\ 13 \rightarrow & 2 \times & P(7,4) \\ 14 \rightarrow & 16 \times & P(7,5) \\ 15 \rightarrow & 16 \times & P(7,5) \\ 16 \rightarrow & 16 \times & P(7,6) \\ 17 \rightarrow & 16 \times & P(7,7) \\ 18 \rightarrow & 64 \times & P(7,7) \\ 19 \rightarrow & 16 \times & P(7,8) \\ 20 \rightarrow & 4 \times & P(7,9) \\ 21 \rightarrow & 16 \times & P(7,10) \\ & & \end{array}$




$\begin{array}{lrl}22 \rightarrow & 32 \times & P(7,17) \\ 23 \rightarrow & 2 \times & P(7,19) \\ 24 \rightarrow & 4 \times & P(7,22) \\ 25 \rightarrow & 32 \times & P(7,24) \\ 26 \rightarrow & 32 \times & P(7,28) \\ 27 \rightarrow & 16 \times & P(7,29)\end{array}$

Nombre total de voisines : 444

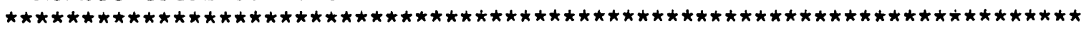
Forme parfaite $P(7,10)$.

$\begin{array}{lllllllll}4 & 1 & 2 & 2 & 0 & 2 & 2 & \text { Nombre de vecteurs minimaux } & : 32 \\ 1 & 4 & 1 & 2 & 2 & 0 & 2 & \text { Minimum } & : 4 \\ 2 & 1 & 4 & 1 & 2 & 2 & 2 & \text { Déterminant } & : 588 \\ 2 & 2 & 1 & 4 & 1 & 2 & 2 & \text { Déterminant (minimum normalisé à 2) } & : 147 / 32 \\ 0 & 2 & 2 & 1 & 4 & 1 & 2 & \text { Ordre du groupe des automorphismes } & : 64 \\ 2 & 0 & 2 & 2 & 1 & 4 & 2 & & \\ 2 & 2 & 2 & 2 & 2 & 2 & 4 & & \end{array}$

Liste des orbites des voisines de la forme $P(7,10)$ :

\begin{tabular}{|c|c|c|c|}
\hline $\begin{array}{l}1 \\
2 \\
3 \\
4 \\
5 \\
6 \\
7 \\
8\end{array}$ & $\begin{array}{l}\rightarrow \\
\Rightarrow \\
\Rightarrow \\
\Rightarrow \\
\Rightarrow \\
\Rightarrow \\
\Rightarrow \\
\Rightarrow\end{array}$ & $\begin{array}{rl}1 & x \\
8 & x \\
8 & X \\
8 & X \\
16 & X \\
16 x \\
16 x \\
32 x\end{array}$ & $\begin{array}{l}P(7,1) \\
P(7,1) \\
P(7,1) \\
P(7,1) \\
P(7,1) \\
P(7,1) \\
P(7,1) \\
P(7,1)\end{array}$ \\
\hline $\begin{array}{r}9 \\
10\end{array}$ & $\overrightarrow{->}$ & $\begin{array}{l}16 \mathrm{x} \\
16 \mathrm{x}\end{array}$ & $\begin{array}{l}P(7,5) \\
P(7,5)\end{array}$ \\
\hline $\begin{array}{l}11 \\
12 \\
13 \\
14\end{array}$ & $\begin{array}{l}-> \\
-> \\
->\end{array}$ & $\begin{array}{r}8 \mathrm{X} \\
16 \mathrm{X} \\
16 \mathrm{x} \\
32 \mathrm{X}\end{array}$ & $\begin{array}{l}P(7,7) \\
P(7,7) \\
P(7,7) \\
P(7,7)\end{array}$ \\
\hline 15 & $\rightarrow$ & $8 x$ & $P(7,8)$ \\
\hline $\begin{array}{l}16 \\
17\end{array}$ & $\begin{array}{l}-> \\
->\end{array}$ & $\begin{array}{l}16 x \\
16 x\end{array}$ & $\begin{array}{l}P(7,10) \\
P(7,10)\end{array}$ \\
\hline $\begin{array}{l}18 \\
19\end{array}$ & $\begin{array}{l}\rightarrow \\
\rightarrow\end{array}$ & $\begin{array}{l}16 \mathrm{x} \\
16 \mathrm{x}\end{array}$ & $\begin{array}{l}P(7,11) \\
P(7,11)\end{array}$ \\
\hline 20 & $\rightarrow$ & $8 x$ & $P(7,18)$ \\
\hline 21 & $\rightarrow$ & $16 x$ & $P(7,23)$ \\
\hline 22 & $\rightarrow$ & $32 x$ & $P(7,24)$ \\
\hline 23 & $\rightarrow$ & $8 x$ & $P(7,28)$ \\
\hline 24 & $\rightarrow$ & $16 \mathrm{x}$ & $P(7,30)$ \\
\hline
\end{tabular}

Nambre total de voisines : 361 


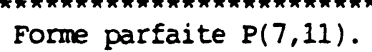

$\begin{array}{rrrrrrr}6 & 1 & 3 & 3 & 3 & 2 & 2 \\ 1 & 6 & 2 & 2 & 2 & -1 & -1 \\ 3 & 2 & 6 & 2 & 2 & -1 & -1 \\ 3 & 2 & 2 & 6 & 0 & 3 & 1 \\ 3 & 2 & 2 & 0 & 6 & 1 & 3 \\ 2 & -1 & -1 & 3 & 1 & 6 & 3 \\ 2 & -1 & -1 & 1 & 3 & 3 & 6\end{array}$

Nombre de vecteurs minimaux

$: 30$

Minimum

: 6

Déterminant

: 10080

Déterminant (minimum normalisé à 2) : 1120/243

Ordre du groupe des automorphismes : 8

Liste des orbites des voisines de la forme $\mathrm{P}(7,11)$ :

$\begin{array}{lll}1 \rightarrow & 2 \times & P(7,1) \\ 2 \rightarrow & 2 \times & P(7,1) \\ 3 \rightarrow & 2 \times & P(7,1) \\ 4 \rightarrow & 2 \times & P(7,1) \\ 5 \rightarrow & 2 X & P(7,1) \\ 6 \rightarrow & 2 \times & P(7,1) \\ 7 \rightarrow & 2 x & P(7,1) \\ 8 \rightarrow & 2 X & P(7,1) \\ 9 \rightarrow & 4 \times & P(7,1)\end{array}$

$10 \rightarrow \quad 1 X \quad P(7,5)$

$11 \rightarrow \quad 1 X \quad P(7,5)$

$12 \rightarrow \quad 4 X \quad P(7,5)$

$13 \rightarrow \quad 4 X \quad P(7,5)$

$14 \rightarrow \quad 2 \times \quad P(7,7)$

$15 \rightarrow \quad 4 X \quad P(7,7)$

$16 \rightarrow \quad 4 X \quad P(7,7)$

$17 \rightarrow \quad 4 X \quad P(7,7)$

$18 \rightarrow \quad 4 X \quad P(7,7)$

$19 \rightarrow \quad 2 X \quad P(7,10)$

$20 \rightarrow \quad 2 X \quad P(7,10)$

$21 \rightarrow \quad 1 X \quad P(7,11)$

$22 \rightarrow \quad 2 X \quad P(7,11)$

$23 \rightarrow \quad 4 X \quad P(7,11)$

$24 \rightarrow \quad 4 \mathrm{X} \quad \mathrm{P}(7,11)$

$25 \rightarrow \quad 2 \times \quad P(7,13)$

$26 \rightarrow \quad 4 X \quad P(7,14)$

$27 \rightarrow \quad 2 \times \quad P(7,15)$

$28 \rightarrow \quad 2 \times \quad P(7,17)$

$29 \rightarrow \quad 4 \times \quad P(7,18)$

$30 \rightarrow \quad 1 X \quad P(7,23)$

$31 \rightarrow \quad 4 \times \quad P(7,24)$

Nombre total de voisines : 82 
Liste des orbites des voisines de la forme $P(7,14)$ :

$\begin{array}{rll}1 \rightarrow & 2 \times & P(7,1) \\ 2 \rightarrow & 2 \times & P(7,1) \\ 3 \rightarrow & 2 \times & P(7,1) \\ 4 \rightarrow & 2 \times & P(7,1) \\ 5 \rightarrow & 2 \times & P(7,1) \\ 6 \rightarrow & 2 \times & P(7,1) \\ 7 \rightarrow & 2 \times & P(7,1) \\ 8 \rightarrow & 1 \times & P(7,5) \\ 9 \rightarrow & 2 \times & P(7,5) \\ 10 \rightarrow & 2 \times & P(7,5) \\ 11 \rightarrow & 1 \times & P(7,6) \\ 12 \rightarrow & 1 \times x & P(7,7) \\ 13 \rightarrow & 2 \times & P(7,7) \\ 14 \rightarrow & 4 \times & P(7,7) \\ 15 \rightarrow & 4 \times & P(7,7) \\ 16 \rightarrow & 4 \times & P(7,7) \\ 17 \rightarrow & 4 \times & P(7,11) \\ 18 \rightarrow & 4 \times & P(7,14) \\ 19 \rightarrow & 2 \times & P(7,15) \\ 20 \rightarrow & 1 \times & P(7,16) \\ 21 \rightarrow & 2 \times & P(7,18) \\ 22 \rightarrow & 1 \times & P(7,21) \\ 23 \rightarrow & 2 \times & P(7,22) \\ 24 \rightarrow & 2 \times & P(7,25)\end{array}$

Nombre total de voisines : 53

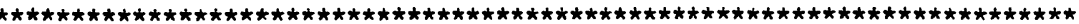
Forme parfaite $P(7,16)$.

$\begin{array}{lllllll}6 & 1 & 1 & 2 & 3 & 3 & 4 \\ 1 & 6 & 1 & 1 & 2 & 3 & 4 \\ 1 & 1 & 6 & 1 & 1 & 2 & 4 \\ 2 & 1 & 1 & 6 & 1 & 1 & 4 \\ 3 & 2 & 1 & 1 & 6 & 1 & 4 \\ 3 & 3 & 2 & 1 & 1 & 6 & 4 \\ 4 & 4 & 4 & 4 & 4 & 4 & 8\end{array}$

Nombre de vecteurs minimaux

: 30

Minimm

$: 6$

Déterminant

: 10368

Déterminant (minimm normalisé à 2) : 128/27

Ordre du groupe des automorphismes : 48

Liste des orbites des voisines de la forme $P(7,16)$ :
$1 \rightarrow$
$2 \rightarrow$
$6 \times \quad P(7,1)$
$3 \rightarrow$
$6 \times \quad P(7,1)$
$12 \times \quad P(7,1)$ 


$\begin{array}{rrr}4 \rightarrow & 6 \times & P(7,5) \\ 5 \rightarrow & 6 \times & P(7,6) \\ 6 \rightarrow & 2 \times & P(7,7) \\ 7 \rightarrow & 12 \times & P(7,7) \\ 8 \rightarrow & 6 \times & P(7,14) \\ 9 \rightarrow & 6 \times & P(7,18) \\ 10 \rightarrow & 6 \times & P(7,22) \\ 11 \rightarrow & 12 \times & P(7,25) \\ 12 \rightarrow & 2 \times & P(7,27)\end{array}$

Nombre total de voisines : 82

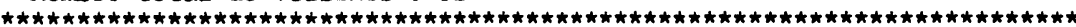
Forme parfaite $P(7,22)$.

$\begin{array}{rrrrrrr}4 & 2 & 2 & 1 & 1 & 2 & 0 \\ 2 & 4 & 1 & 2 & 2 & 0 & 2 \\ 2 & 1 & 4 & 2 & -1 & 2 & 0 \\ 1 & 2 & 2 & 4 & 1 & 0 & 2 \\ 1 & 2 & -1 & 1 & 4 & 0 & 2 \\ 2 & 0 & 2 & 0 & 0 & 4 & -1 \\ 0 & 2 & 0 & 2 & 2 & -1 & 4\end{array}$

Nombre de vecteurs minimaux

: 30

Minimum

$: 4$

Déteminant

630

Déterminant (minimum normalisé à 2) : 315/64

Ordre du groupe des automorphismes : 32

Liste des orbites des voisines de la forme $P(7,22)$ :

$\begin{array}{rrr}1 \rightarrow & 1 \times & P(7,1) \\ 2 \rightarrow & 4 \times & P(7,1) \\ 3 \rightarrow & 4 \times & P(7,1) \\ 4 \rightarrow & 4 \times & P(7,1) \\ 5 \rightarrow & 8 \times & P(7,1) \\ 6 \rightarrow & 8 \times & P(7,1) \\ 7 \rightarrow & 8 \times & P(7,1) \\ 8 \rightarrow & 2 \times & P(7,3) \\ 9 \rightarrow & 2 \times & P(7,4) \\ 10 \rightarrow & 8 \times & P(7,7) \\ 11 \rightarrow & 8 \times & P(7,7) \\ 12 \rightarrow & 16 \times & P(7,7) \\ 13 \rightarrow & 1 \times & P(7,8) \\ 14 \rightarrow & 8 \times & P(7,14) \\ 15 \rightarrow & 4 \times & P(7,16) \\ 16 \rightarrow & 8 \times & P(7,22) \\ 17 \rightarrow & 4 \times & P(7,25) \\ 18 \rightarrow & 1 \times & P(7,27) \\ 19 \rightarrow & 2 \times & P(7,29) \\ 20 \rightarrow & 8 \times & P(7,31)\end{array}$

Nombre total de voisines : 109 
Forme parfaite $P(7,26)$.

$\begin{array}{lllllll}4 & 1 & 2 & 2 & 2 & 2 & 2 \\ 1 & 4 & 2 & 2 & 2 & 2 & 2 \\ 2 & 2 & 4 & 1 & 1 & 2 & 2 \\ 2 & 2 & 1 & 4 & 1 & 2 & 2 \\ 2 & 2 & 1 & 1 & 4 & 2 & 2 \\ 2 & 2 & 2 & 2 & 2 & 4 & 2 \\ 2 & 2 & 2 & 2 & 2 & 2 & 4\end{array}$

Nombre de vecteurs minimaux

$: 30$

Minimum

$: 4$

Déteminant

$: 648$

Déterminant (minimum normalisé à 2) : $81 / 16$

Ordre du groupe des automorphismes : 864

Liste des orbites des voisines de la fome $P(7,26)$ :
$1 \rightarrow$
$2 \rightarrow$
$9 \times \quad P(7,1)$
$3 \rightarrow$
$12 \times \quad P(7,1)$
$4 \rightarrow$
$3 \times \quad P(7,3)$
$5 \rightarrow$
$6 \times \quad P(7,4)$
$6 \rightarrow$
$27 \times \quad P(7,29)$

Nombre total de voisines : 93

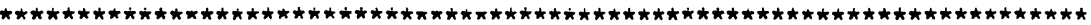

Forme parfaite $P(7,27)$.

$\begin{array}{lllllll}4 & 1 & 2 & 2 & 2 & 2 & 3 \\ 1 & 4 & 2 & 2 & 2 & 2 & 3 \\ 2 & 2 & 4 & 1 & 2 & 2 & 3 \\ 2 & 2 & 1 & 4 & 2 & 2 & 3 \\ 2 & 2 & 2 & 2 & 4 & 1 & 3 \\ 2 & 2 & 2 & 2 & 1 & 4 & 3 \\ 3 & 3 & 3 & 3 & 3 & 3 & 6\end{array}$

Nombre de vecteurs minimaux

Minimum

Déterminant

: 30

$: 4$

Déterminant (minimum normalisé à 2): $81 / 6$

ordre du groupe des automorphismes : 768

Liste des orbites des voisines de la forme $P(7,27)$ :

$\begin{array}{lrl}1 \rightarrow & 24 \times & P(7,1) \\ 2 \rightarrow & 32 \times & P(7,1) \\ 3 \rightarrow & 96 \times & P(7,1) \\ 4 \rightarrow & 4 \times & P(7,4) \\ 5 \rightarrow & 192 \times & P(7,7) \\ 6 \rightarrow & 32 \times & P(7,16) \\ 7 \rightarrow & 24 \times & P(7,22) \\ 8 \rightarrow & 96 \times & P(7,25) \\ 9 \rightarrow & 64 \times & P(7,28) \\ 10 \rightarrow & 4 \times & P(7,32)\end{array}$

Nombre total de voisines : 568 
Forme parfaite $\mathrm{P}(7,28)$.

$\begin{array}{rrrrrrr}4 & 1 & 2 & 2 & 2 & 1 & 5 \\ 1 & 4 & 1 & 2 & 2 & 2 & 5 \\ 2 & 1 & 4 & 1 & 2 & 2 & 5 \\ 2 & 2 & 1 & 4 & 1 & 2 & 5 \\ 2 & 2 & 2 & 1 & 4 & 1 & 5 \\ 1 & 2 & 2 & 2 & 1 & 4 & 5 \\ 5 & 5 & 5 & 5 & 5 & 5 & 14\end{array}$

Nombre de vecteurs minimaux

: 30

Minimam

$: 4$

Déterminant

: 648

DÉterminant (minimum normalisé à 2) : 81/16

Ordre du groupe des automorphismes : 24

Liste des orbites des voisines de la forme $P(7,28)$ :

$\begin{array}{lrl}1 \rightarrow & 1 \times & P(7,1) \\ 2 \rightarrow & 3 \times & P(7,1) \\ 3 \rightarrow & 6 \times & P(7,1) \\ 4 \rightarrow & 6 \times & P(7,1) \\ 5 \rightarrow & 12 \times & P(7,1) \\ 6 \rightarrow & 1 \times & P(7,7) \\ 7 \rightarrow & 6 \times & P(7,7) \\ 8 \rightarrow & 6 \times & P(7,7) \\ 9 \rightarrow & 6 \times & P(7,7) \\ 10 \rightarrow & 6 \times & P(7,8) \\ 11 \rightarrow & 3 \times & P(7,10) \\ 12 \rightarrow & 6 \times & P(7,24) \\ 13 \rightarrow & 6 \times & P(7,25) \\ 14 \rightarrow & 2 \times & P(7,27) \\ 15 \rightarrow & 6 \times & P(7,28) \\ 16 \rightarrow & 6 \times & P(7,29)\end{array}$

Nomize total de voisines : 82 


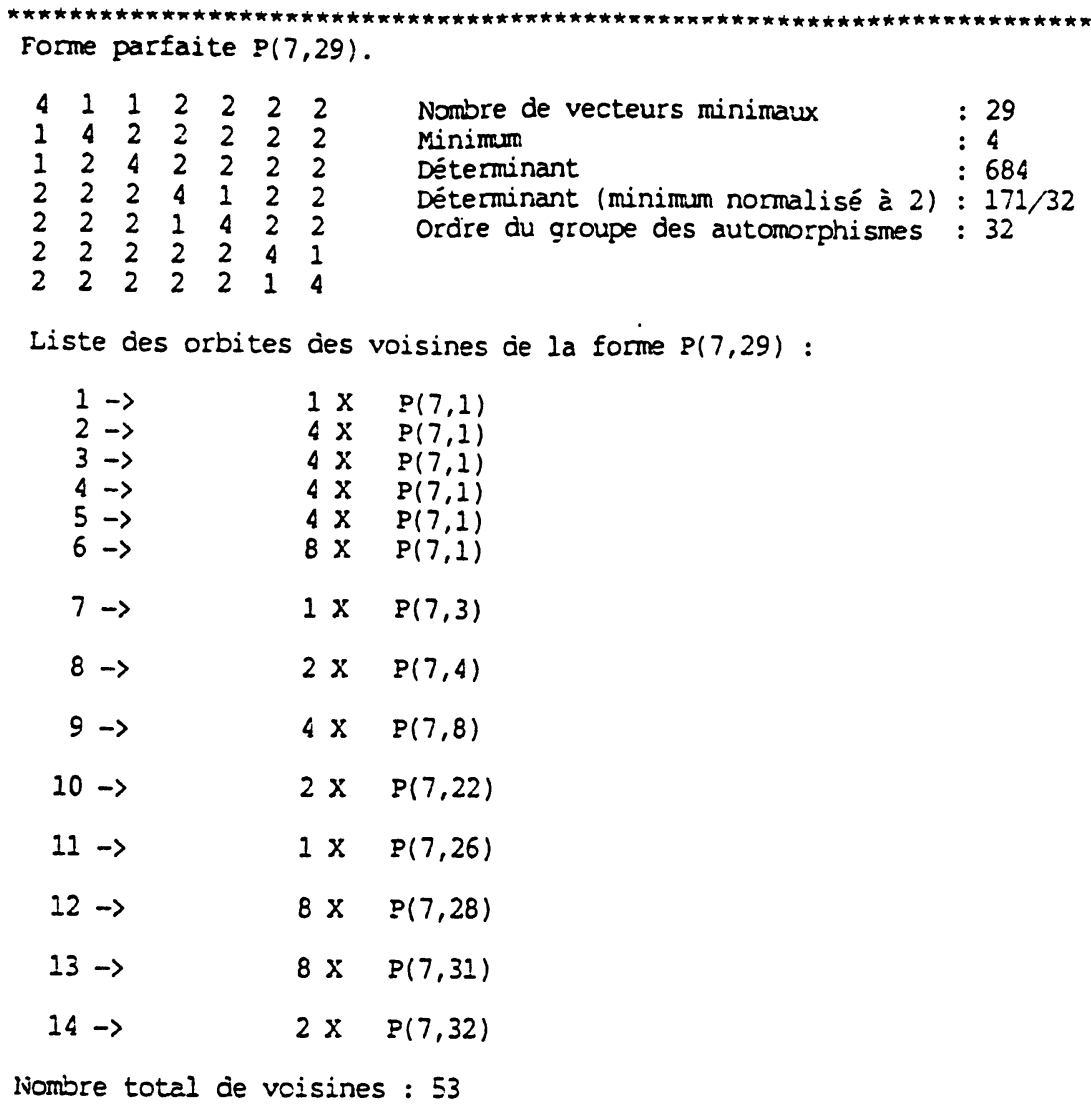

Institut de Mathématiques et d'Informatique (IMI) Chantemerle 20 - Case postale 2 2007 NEUCHÂTEL SUISSE. 